\title{
Theoretical Study of the Copper Complexes with Aminoguanidine: Investigating Secondary Antioxidant Activity
}

\author{
Guillermo García-Díez, ${ }^{1}$ Rafael Ramis, ${ }^{2 a}$ Nelaine Mora-Diez ${ }^{1 *}$ \\ ${ }^{1}$ Thompson Rivers University, Department of Chemistry, Kamloops, B.C., V2C 0C8 Canada. \\ ${ }^{2}$ Universitat de les Illes Balears, Departament de Química, 07122 Palma de Mallorca, Spain.
}

\section{Electronic Supplementary Information}

(32 pages)

\section{Contents:}

Table S1. Absolute enthalpies and Gibbs free energies of the different species considered in this study at the M05(SMD)/6-311+G(d,p) level of theory in water at $298.15 \mathrm{~K}$.

Table S2. Standard Gibbs free energy of reaction $\left(\Delta \mathrm{G}^{\circ}, \mathrm{kcal} / \mathrm{mol}\right)$ and activation $\left(\Delta \mathrm{G}^{\neq}, \mathrm{kcal} / \mathrm{mol}\right)$, and various rate constants $\left(\mathrm{k}, \mathrm{kD}\right.$ and $\left.\mathrm{kapp}_{\mathrm{a}} \mathrm{M}^{-1} \mathrm{~s}^{-1}\right)$ for the initial reaction of the Haber-Weiss cycle (with and without copper complexation with $\mathrm{AG}$ or $\mathrm{AGH}^{+}$) in aqueous solution at $298.15 \mathrm{~K}$.

Figure S1. Optimized geometries of $\mathrm{Cu}(\mathrm{I})$ complexes with $\mathrm{AG}$ and $\mathrm{AGH}^{+}$in aqueous solution that appear in Table S2, but are not shown on Figure 5 (indicating the $\mathrm{Cu}$ (II) complex used as starting point in each case; bond distances in $\AA$ ).

M05(SMD)/6-311+G(d,p) Cartesian coordinates of the optimized geometries in water of the species calculated in this study.

a Author contributed to this paper while visiting TRU as a graduate student for two months in 2018.

* Corresponding author e-mail: nmora@tru.ca 
Table S1. Absolute enthalpies and Gibbs free energies of the different species considered in this study at the M05(SMD)/6-311+G(d,p) level of theory in water at $298.15 \mathrm{~K}$.

\begin{tabular}{|c|c|c|c|}
\hline & Species & $\mathrm{H}^{\circ}(\mathrm{au})$ & $\mathrm{G}^{\circ}(\mathrm{au})$ \\
\hline [1] & {$[\mathrm{Cu}(\mathrm{AGH}) 2]^{4+}$} & -2162.137164 & -2162.192466 \\
\hline$[2]$ & {$\left[\mathrm{Cu}(\mathrm{AGH})_{2}\left(\mathrm{H}_{2} \mathrm{O}\right)_{2}\right]^{4+}$} & -2314.986229 & -2315.054751 \\
\hline$[3]$ & {$\left[\mathrm{Cu}(\mathrm{AGH})\left(\mathrm{H}_{2} \mathrm{O}\right)_{2}\right]^{3+}$} & -2053.993037 & -2054.044106 \\
\hline$[4]$ & {$\left[\mathrm{Cu}(\mathrm{AGH})\left(\mathrm{H}_{2} \mathrm{O}\right)_{3}\right]^{3+}$} & -2130.416463 & -2130.473843 \\
\hline$[5]$ & {$\left[\mathrm{Cu}\left(\mathrm{AG}_{\mathrm{A}}\right)\left(\mathrm{H}_{2} \mathrm{O}\right)_{2}\right]^{2+}$} & -2053.556005 & -2053.607736 \\
\hline [6] & {$\left[\mathrm{Cu}\left(\mathrm{AG}_{\mathrm{A}}\right)\left(\mathrm{H}_{2} \mathrm{O}\right)_{3}\right]^{2+}$} & -2129.981156 & -2130.039793 \\
\hline [7] & {$\left[\mathrm{Cu}(\mathrm{AGB})\left(\mathrm{H}_{2} \mathrm{O}\right)_{2}\right]^{2+}$} & -2053.557068 & -2053.607508 \\
\hline [8] & {$\left[\mathrm{Cu}\left(\mathrm{AG}_{\mathrm{B}}\right)\left(\mathrm{H}_{2} \mathrm{O}\right)_{3}\right]^{2+}$} & -2129.979191 & -2130.038052 \\
\hline [9] & {$\left[\mathrm{Cu}\left(\mathrm{AGC}_{\mathrm{C}}\right)\left(\mathrm{H}_{2} \mathrm{O}\right)_{2}\right]^{2+}$} & -2053.557678 & -2053.607844 \\
\hline$[10]$ & {$\left[\mathrm{Cu}\left(\mathrm{AGC}_{\mathrm{C}}\right)\left(\mathrm{H}_{2} \mathrm{O}\right)_{3}\right]^{2+}$} & -2129.981784 & -2130.039749 \\
\hline$[11]$ & {$\left[\mathrm{Cu}(\mathrm{AGD})\left(\mathrm{H}_{2} \mathrm{O}\right)_{2}\right]^{2+}$} & -2053.584492 & -2053.636206 \\
\hline [12] & {$\left[\mathrm{Cu}\left(\mathrm{AG}_{\mathrm{A}}\right)_{2}\right]^{2+}$} & -2161.265479 & -2161.322151 \\
\hline$[13]$ & {$\left[\mathrm{Cu}\left(\mathrm{AG}_{\mathrm{A}}\right)_{2}\right]^{2+}$ (mirror image) } & -2161.264020 & -2161.321601 \\
\hline$[14]$ & {$\left[\mathrm{Cu}\left(\mathrm{AG}_{\mathrm{A}}\right)_{2}\left(\mathrm{H}_{2} \mathrm{O}\right)_{2}\right]^{2+}$} & -2314.116308 & -2314.186989 \\
\hline$[15]$ & {$\left[\mathrm{Cu}\left(\mathrm{AG}_{\mathrm{B}}\right)_{2}\right]^{2+}$} & -2161.268668 & -2161.322422 \\
\hline$[16]$ & $\left[\mathrm{Cu}\left(\mathrm{AG}_{\mathrm{B}}\right)_{2}\right]^{2+}$ (mirror image $)$ & -2161.267691 & -2161.322038 \\
\hline [17] & {$\left[\mathrm{Cu}\left(\mathrm{AG}_{\mathrm{B}}\right)_{2}\left(\mathrm{H}_{2} \mathrm{O}\right)\right]^{2+}$} & -2237.692754 & -2237.754323 \\
\hline [18] & {$\left[\mathrm{Cu}\left(\mathrm{AG}_{\mathrm{B}}\right)_{2}\left(\mathrm{H}_{2} \mathrm{O}\right)_{2}\right]^{2+}$} & -2314.115582 & -2314.185939 \\
\hline [19] & {$[\mathrm{Cu}(\mathrm{AGc}) 2]^{2+}$} & -2161.269377 & -2161.322630 \\
\hline$[20]$ & {$\left[\mathrm{Cu}(\mathrm{AGc})_{2}\right]^{2+}($ mirror image $)$} & -2161.268934 & -2161.322305 \\
\hline$[21]$ & {$\left[\mathrm{Cu}(\mathrm{AGc})_{2}\left(\mathrm{H}_{2} \mathrm{O}\right)\right]^{2+}$} & -2237.693102 & -2237.754814 \\
\hline$[22]$ & {$\left[\mathrm{Cu}\left(\mathrm{AGc}_{\mathrm{C}}\right)_{2}\left(\mathrm{H}_{2} \mathrm{O}\right)_{2}\right]^{2+}$} & -2314.116758 & -2314.186477 \\
\hline$[23]$ & {$\left[\mathrm{Cu}(\mathrm{AGD})_{2}\right]^{2+}$} & -2161.322107 & -2161.375399 \\
\hline$[24]$ & {$[\mathrm{Cu}(\mathrm{AGD}) 2]^{2+}$ (mirror image) } & -2161.321112 & -2161.375573 \\
\hline$[25]$ & {$\left[\mathrm{Cu}(\mathrm{AGD})_{2}\left(\mathrm{H}_{2} \mathrm{O}\right)\right]^{2+}$} & -2237.717374 & -2237.778429 \\
\hline$[26]$ & {$\left[\mathrm{Cu}\left(\mathrm{AG}_{\mathrm{D}}\right)_{2}\left(\mathrm{H}_{2} \mathrm{O}\right)_{2}\right]^{2+}$} & -2314.112834 & -2314.182675 \\
\hline [27] & {$\left[\mathrm{Cu}\left(\mathrm{AG}_{\mathrm{A}}\right)\left(\mathrm{AG}_{\mathrm{D}}\right)\right]^{2+}$} & -2161.294110 & -2161.350350 \\
\hline$[28]$ & {$\left[\mathrm{Cu}\left(\mathrm{AG}_{\mathrm{B}}\right)\left(\mathrm{AG}_{\mathrm{D}}\right)\right]^{2+}$} & -2161.294993 & -2161.348891 \\
\hline [29] & {$\left[\mathrm{Cu}\left(\mathrm{AG}_{\mathrm{C}}\right)\left(\mathrm{AG}_{\mathrm{D}}\right)\right]^{2+}$} & -2161.295878 & -2161.351553 \\
\hline$[30]$ & {$\left[\mathrm{Cu}\left(\mathrm{AGc}_{\mathrm{C}}\right)\left(\mathrm{AG}_{\mathrm{D}}\right)\right]^{2+}$ (mirror image) } & -2161.295629 & -2161.349231 \\
\hline$[31]$ & {$\left[\mathrm{Cu}(\mathrm{AGH})\left(\mathrm{H}_{2} \mathrm{O}\right)\right]^{2+} \cdot 2 \mathrm{H}_{2} \mathrm{O}$} & -2130.599152 & -2130.664595 \\
\hline$[32]$ & {$\left[\mathrm{Cu}\left(\mathrm{AG}_{\mathrm{C}}\right)\left(\mathrm{H}_{2} \mathrm{O}\right)\right]^{+} \cdot \mathrm{H}_{2} \mathrm{O}$} & -2053.730745 & -2053.786308 \\
\hline [33] & {$\left[\mathrm{Cu}(\mathrm{AGc})\left(\mathrm{H}_{2} \mathrm{O}\right)\right]^{+} \cdot 2 \mathrm{H}_{2} \mathrm{O}$} & -2130.161639 & -2130.227268 \\
\hline [34] & {$\left[\mathrm{Cu}(\mathrm{AGc})_{2}\right]^{+} \cdot 2 \mathrm{H}_{2} \mathrm{O}$} & -2314.296160 & -2314.370854 \\
\hline [35] & {$\left[\mathrm{Cu}\left(\mathrm{AG}_{\mathrm{A}}\right)_{2}\right]^{+} \cdot 2 \mathrm{H}_{2} \mathrm{O}$} & -2314.297449 & -2314.373896 \\
\hline$[36]$ & {$\left[\mathrm{Cu}(\mathrm{AGc})_{2}\right]^{+}$(mirror image) } & -2161.441643 & -2161.498475 \\
\hline [37] & {$\left[\mathrm{Cu}(\mathrm{AGC})_{2}\right]^{+}$} & -2161.441835 & -2161.500144 \\
\hline
\end{tabular}




\begin{tabular}{|c|c|c|c|}
\hline [38] & {$\left[\mathrm{Cu}\left(\mathrm{AG}_{\mathrm{D}}\right)\left(\mathrm{H}_{2} \mathrm{O}\right)\right]^{+} \cdot \mathrm{H}_{2} \mathrm{O}$} & -2053.750025 & -2053.805633 \\
\hline [39] & {$[\mathrm{Cu}(\mathrm{AG})(\mathrm{AG})]^{+}$} & -2161.458647 & -2161.518008 \\
\hline [40] & {$\left[\mathrm{Cu}(\mathrm{AGD})_{2}\right]^{+} \cdot \mathrm{H}_{2} \mathrm{O}$} & -2237.877719 & -2237.946507 \\
\hline [41] & {$\left[\mathrm{Cu}(\mathrm{AGD})_{2}\right]^{+}$} & -2161.741105 & -2161.530591 \\
\hline [42] & {$\left[\mathrm{Cu}(\mathrm{AGD})_{2}\right]^{+}$(mirror image) } & -2161.471544 & -2161.531491 \\
\hline [43] & {$\left[\mathrm{Cu}\left(\mathrm{H}_{2} \mathrm{O}\right)_{2}\right]^{+} \cdot 2 \mathrm{H}_{2} \mathrm{O}$} & -1946.026769 & -1946.081005 \\
\hline [44] & {$\left[\mathrm{Cu}\left(\mathrm{H}_{2} \mathrm{O}\right)_{4}\right]^{2+}$} & -1945.844939 & -1945.891638 \\
\hline [45] & $\mathrm{AGH}^{+}$ & -260.984009 & -261.021567 \\
\hline [46] & $\left(\mathrm{AGH}^{+}\right)_{2}$ & -521.968983 & -522.026679 \\
\hline [47] & $\mathrm{AG}_{\mathrm{A}}$ & -260.521678 & -260.556867 \\
\hline [48] & $\mathrm{AG}_{\mathrm{B}}$ & -260.521390 & -260.556485 \\
\hline [49] & $\mathrm{AG}_{\mathrm{C}}$ & -260.521218 & -260.556191 \\
\hline [50] & $\mathrm{AG}_{\mathrm{D}}$ & -260.517382 & -260.553434 \\
\hline [51] & $\left(\mathrm{AG}_{\mathrm{A}}\right)_{2}$ & -521.049437 & -521.103032 \\
\hline [52] & $\left(\mathrm{AG}_{\mathrm{B}}\right)_{2}$ & -521.044038 & -521.100221 \\
\hline [53] & $(\mathrm{AGc}) 2$ & -521.044308 & -521.099285 \\
\hline [54] & $\left(A G_{D}\right)_{2}$ & -521.037665 & -521.092705 \\
\hline [55] & $\left(A G_{C}\right)\left(A_{D}\right)$ & -521.040469 & -521.095685 \\
\hline
\end{tabular}


Table S2. Standard Gibbs free energy of reaction $\left(\Delta \mathrm{G}^{\circ}, \mathrm{kcal} / \mathrm{mol}\right)$ and activation $\left(\Delta \mathrm{G}^{\neq}, \mathrm{kcal} / \mathrm{mol}\right)$, and various rate constants (k, $\mathrm{kD}$ and $\mathrm{k}_{\mathrm{app}}, \mathrm{M}^{-1} \mathrm{~s}^{-1}$ ) for the initial reaction of the Haber-Weiss cycle (with and without copper complexation with AG or AGH+) in aqueous solution at $298.15 \mathrm{~K}$.

\begin{tabular}{|c|c|c|c|c|c|}
\hline Reaction & $\Delta \mathrm{G}^{0}$ & $\Delta \mathrm{G}^{\neq}$ & $\mathrm{k}$ & $\mathrm{kb}$ & kapp \\
\hline$\left[\mathrm{Cu}\left(\mathrm{H}_{2} \mathrm{O}\right)_{4}\right]^{2+}+\mathrm{O}_{2}^{--} \rightarrow\left[\mathrm{Cu}\left(\mathrm{H}_{2} \mathrm{O}\right)_{2}\right]^{+} \cdot 2 \mathrm{H}_{2} \mathrm{O}+\mathrm{O}_{2}$ & -32.6 & 0.2 & $4.37 \times 10^{12}$ & $7.73 \times 10^{9}$ & $7.71 \times 10^{9}$ \\
\hline$[4]\left[\mathrm{Cu}(\mathrm{AGH})\left(\mathrm{H}_{2} \mathrm{O}\right)_{3}\right]^{3+}+\mathrm{O}_{2} \cdot-[31]\left[\mathrm{Cu}(\mathrm{AGH})\left(\mathrm{H}_{2} \mathrm{O}\right)\right]^{2+} \cdot 2 \mathrm{H}_{2} \mathrm{O}+\mathrm{O}_{2}$ & -33.4 & 0.1 & $5.50 \times 10^{12}$ & $7.68 \times 10^{9}$ & $7.67 \times 10^{9}$ \\
\hline$[9]\left[\mathrm{Cu}(\mathrm{AGc})\left(\mathrm{H}_{2} \mathrm{O}\right)_{2}\right]^{2+}+\mathrm{O}_{2} \cdot \rightarrow[32]\left[\mathrm{Cu}(\mathrm{AGc})\left(\mathrm{H}_{2} \mathrm{O}\right)\right]^{+} \cdot \mathrm{H}_{2} \mathrm{O}+\mathrm{O}_{2}$ & -25.7 & 0.4 & $3.02 \times 10^{12}$ & $7.69 \times 10^{9}$ & $7.67 \times 10^{9}$ \\
\hline$[10]\left[\mathrm{Cu}(\mathrm{AGc})\left(\mathrm{H}_{2} \mathrm{O}\right)_{3}\right]^{2+}+\mathrm{O}_{2} \cdot-[33]\left[\mathrm{Cu}(\mathrm{AGc})\left(\mathrm{H}_{2} \mathrm{O}\right)\right]^{+} \cdot 2 \mathrm{H}_{2} \mathrm{O}+\mathrm{O}_{2}$ & -31.4 & 0.5 & $2.61 \times 10^{12}$ & $7.84 \times 10^{9}$ & $7.82 \times 10^{9}$ \\
\hline$[22]\left[\mathrm{Cu}(\mathrm{AGc})_{2}\left(\mathrm{H}_{2} \mathrm{O}\right)_{2}\right]^{2+}+\mathrm{O}_{2}-\cdots[34]\left[\mathrm{Cu}(\mathrm{AGc})_{2}\right]^{+} \cdot 2 \mathrm{H}_{2} \mathrm{O}+\mathrm{O}_{2}$ & -29.4 & 0.6 & $2.37 \times 10^{12}$ & $7.99 \times 10^{9}$ & $7.96 \times 10^{9}$ \\
\hline$[14]\left[\mathrm{Cu}\left(\mathrm{AG}_{\mathrm{A}}\right)_{2}\left(\mathrm{H}_{2} \mathrm{O}\right)_{2}\right]^{2+}+\mathrm{O}_{2}-\cdots[35]\left[\mathrm{Cu}\left(\mathrm{AG}_{\mathrm{A}}\right)_{2}\right]^{+} \cdot 2 \mathrm{H}_{2} \mathrm{O}+\mathrm{O}_{2}$ & -31.0 & 0.6 & $2.22 \times 10^{12}$ & $7.88 \times 10^{9}$ & $7.85 \times 10^{9}$ \\
\hline$[20]\left[\mathrm{Cu}(\mathrm{AGC})_{2}\right]^{2+}+\mathrm{O}_{2}-\rightarrow[36]\left[\mathrm{Cu}(\mathrm{AGc})_{2}\right]^{+}+\mathrm{O}_{2}^{\mathrm{a}}$ & -24.3 & 0.8 & $1.66 \times 10^{12}$ & $7.94 \times 10^{9}$ & $7.90 \times 10^{9}$ \\
\hline$[19]\left[\mathrm{Cu}(\mathrm{AGc})_{2}\right]^{2+}+\mathrm{O}_{2}-\rightarrow[37]\left[\mathrm{Cu}(\mathrm{AGc})_{2}\right]^{+}+\mathrm{O}_{2}$ & -25.1 & 0.8 & $1.52 \times 10^{12}$ & $7.82 \times 10^{9}$ & $7.78 \times 10^{9}$ \\
\hline$[11]\left[\mathrm{Cu}(\mathrm{AGD})\left(\mathrm{H}_{2} \mathrm{O}\right)_{2}\right]^{2+}+\mathrm{O}_{2} \cdot-[38]\left[\mathrm{Cu}(\mathrm{AGD})\left(\mathrm{H}_{2} \mathrm{O}\right)\right]^{+} \cdot \mathrm{H}_{2} \mathrm{O}+\mathrm{O}_{2}$ & -20.0 & 1.8 & $3.03 \times 10^{11}$ & $7.66 \times 10^{9}$ & $7.47 \times 10^{9}$ \\
\hline$[29][\mathrm{Cu}(\mathrm{AGc})(\mathrm{AGD})]^{2+}+\mathrm{O}_{2}-\cdots[39][\mathrm{Cu}(\mathrm{AGc})(\mathrm{AGD})]^{+}+\mathrm{O}_{2}$ & -18.2 & 2.2 & $1.41 \times 10^{11}$ & $7.81 \times 10^{9}$ & $7.40 \times 10^{9}$ \\
\hline$[25]\left[\mathrm{Cu}(\mathrm{AGD})_{2}\left(\mathrm{H}_{2} \mathrm{O}\right)\right]^{2+}+\mathrm{O}_{2}-\rightarrow[40]\left[\mathrm{Cu}(\mathrm{AGD})_{2}\right]^{+} \cdot \mathrm{H}_{2} \mathrm{O}+\mathrm{O}_{2}$ & -19.2 & 2.8 & $5.52 \times 10^{10}$ & $8.04 \times 10^{9}$ & $7.02 \times 10^{9}$ \\
\hline$[23]\left[\mathrm{Cu}(\mathrm{AG})_{2}\right]^{2+}+\mathrm{O}_{2}-\rightarrow[41]\left[\mathrm{Cu}(\mathrm{AGD})_{2}\right]^{+}+\mathrm{O}_{2}$ & -11.1 & 4.1 & $6.28 \times 10^{9}$ & $7.85 \times 10^{9}$ & $3.49 \times 10^{9}$ \\
\hline$[24]\left[\mathrm{Cu}(\mathrm{AG})_{2}\right]^{2+}+\mathrm{O}_{2}-\operatorname{[42]}\left[\mathrm{Cu}\left(\mathrm{AG}_{\mathrm{D}}\right)_{2}\right]^{+}+\mathrm{O}_{2} \mathrm{a}$ & -11.6 & 4.3 & $4.32 \times 10^{9}$ & $7.94 \times 10^{9}$ & $2.80 \times 10^{9}$ \\
\hline
\end{tabular}

a The $\mathrm{Cu}(\mathrm{II})$ complex has the AG ligands in mirror image conformation (see Figure 4). 


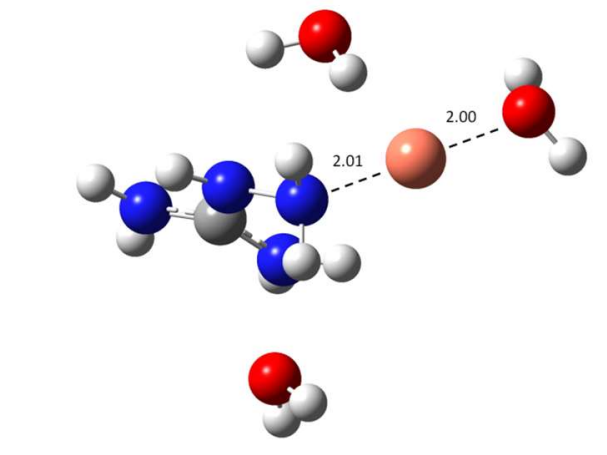

$$
[4] \rightarrow[31]\left[\mathrm{Cu}(\mathrm{AGH})\left(\mathrm{H}_{2} \mathrm{O}\right)\right]^{2+} \cdot 2 \mathrm{H}_{2} \mathrm{O}
$$
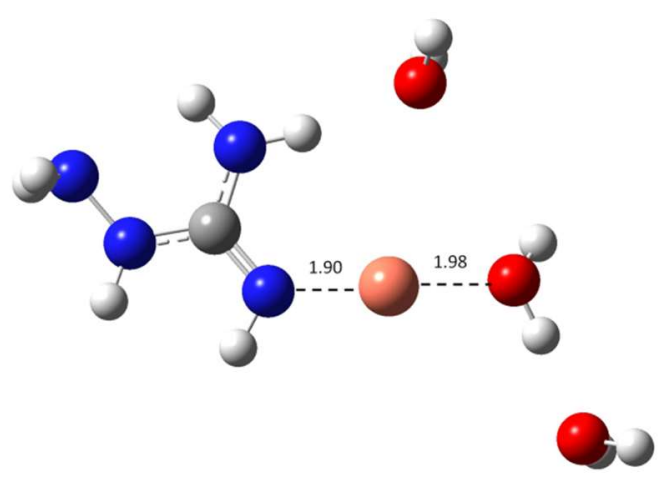

$$
[10] \rightarrow[33]\left[\mathrm{Cu}(\mathrm{AGc})\left(\mathrm{H}_{2} \mathrm{O}\right)\right]^{+} \cdot 2 \mathrm{H}_{2} \mathrm{O}
$$



$$
[14] \rightarrow[35]\left[\mathrm{Cu}\left(\mathrm{AG}_{\mathrm{A}}\right)_{2}\right]^{+} \cdot 2 \mathrm{H}_{2} \mathrm{O}
$$
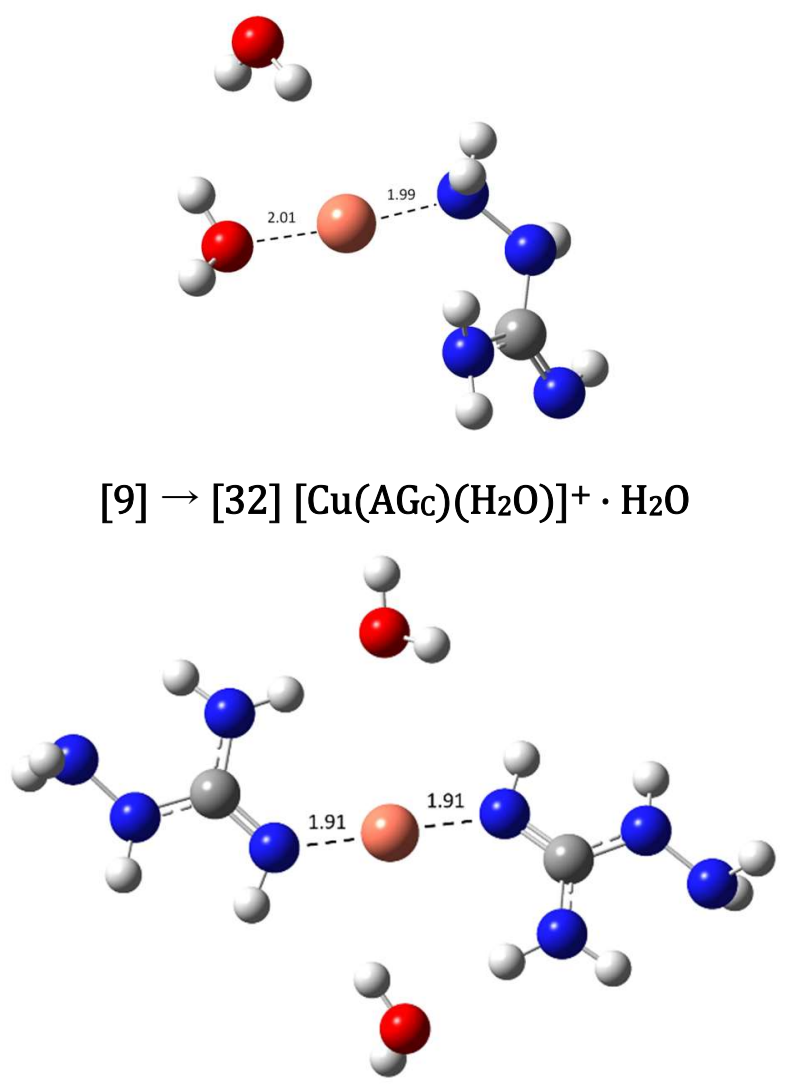

$$
[22] \rightarrow[34]\left[\mathrm{Cu}(\mathrm{AGc})_{2}\right]^{+} \cdot 2 \mathrm{H}_{2} \mathrm{O}
$$



$[20] \rightarrow[36]\left[\mathrm{Cu}(\mathrm{AGc})_{2}\right]^{+}$(mirror image) 


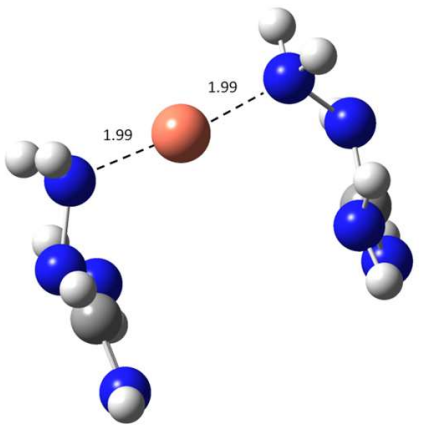

$[19] \rightarrow[37]\left[\mathrm{Cu}(\mathrm{AGc})_{2}\right]^{+}$

Figure S1. Optimized geometries of $\mathrm{Cu}(\mathrm{I})$ complexes with $\mathrm{AG}$ and $\mathrm{AGH}^{+}$in aqueous solution that appear in Table S1, but are not shown on Figure 5 (indicating the $\mathrm{Cu}(\mathrm{II})$ complex used as starting point in each case; bond distances in $\AA$ ). 


\section{M05(SMD)/6-311+G(d,p) Cartesian coordinates of the optimized geometries in water of the species calculated in this study.}

\section{$[1]\left[\mathrm{Cu}(\mathrm{AGH})_{2}\right]^{4+}$}

\begin{abstract}
Charge $=4$ Multiplicity $=2$
$\mathrm{Cu}, 0,0.0004639186,0.0037826539,0.0443004261$ $\mathrm{N}, 0,1.336640599,-1.5660710321,0.090370809$ $\mathrm{H}, 0,1.2318632871,-2.0964320247,0.9545519971$ $\mathrm{H}, 0,1.1650185989,-2.1944479722,-0.6936893387$ $\mathrm{N}, 0,1.7609952073,1.0626944337,-0.09424889$ $\mathrm{H}, 0,1.8436175571,1.7784358118,0.6300771848$ C, $0,2.8830394312,0.2087353266,-0.0472088069$ $\mathrm{N}, 0,2.6404718807,-1.086479422,0.0084096217$ $\mathrm{H}, 0,3.4035300459,-1.7569328911,0.0282451597$ $\mathrm{N}, 0,4.0918497189,0.6925135106,-0.0647970597$ $\mathrm{H}, \mathrm{O}, 4.2369419957,1.6904958376,-0.134020204$ $\mathrm{H}, 0,4.9002040114,0.0849320251,-0.0215655666$ $\mathrm{N}, 0,-1.3405637606,1.5734697254,0.0151019205$ $\mathrm{H}, 0,-1.214447759,2.1581856666,0.8405185709$ $\mathrm{H}, \mathrm{O},-1.1924686253,2.1509879373,-0.8116552431$ $\mathrm{N}, 0,-2.6444394885,1.0869773669,-0.0037450992$ $\mathrm{C}, 0,-2.8805170673,-0.2107833322,0.0142227272$ $\mathrm{N}, 0,-1.7628024905,-1.056748742,0.1656802413$ $\mathrm{N}, 0,-4.080318188,-0.7049920467,-0.0963806171$ $\mathrm{H}, \mathrm{O},-4.2244909477,-1.7034670755,-0.0337785165$ $\mathrm{H}, \mathrm{O},-4.8844163414,-0.1041645311,-0.2274692143$ $\mathrm{H}, \mathrm{O},-1.8228671833,-1.8308625214,-0.4973347339$ $\mathrm{H}, \mathrm{O},-3.4079790279,1.7530355935,-0.0759936348$ $\mathrm{H}, \mathrm{O},-1.7869934799,-1.4696935623,1.1022181728$ $\mathrm{H}, 0,1.7603811074,1.556871264,-0.9904309063$
\end{abstract}

\section{[2] $\left[\mathrm{Cu}(\mathrm{AGH})_{2}\left(\mathrm{H}_{2} \mathrm{O}\right)_{2}\right]^{4+}$}
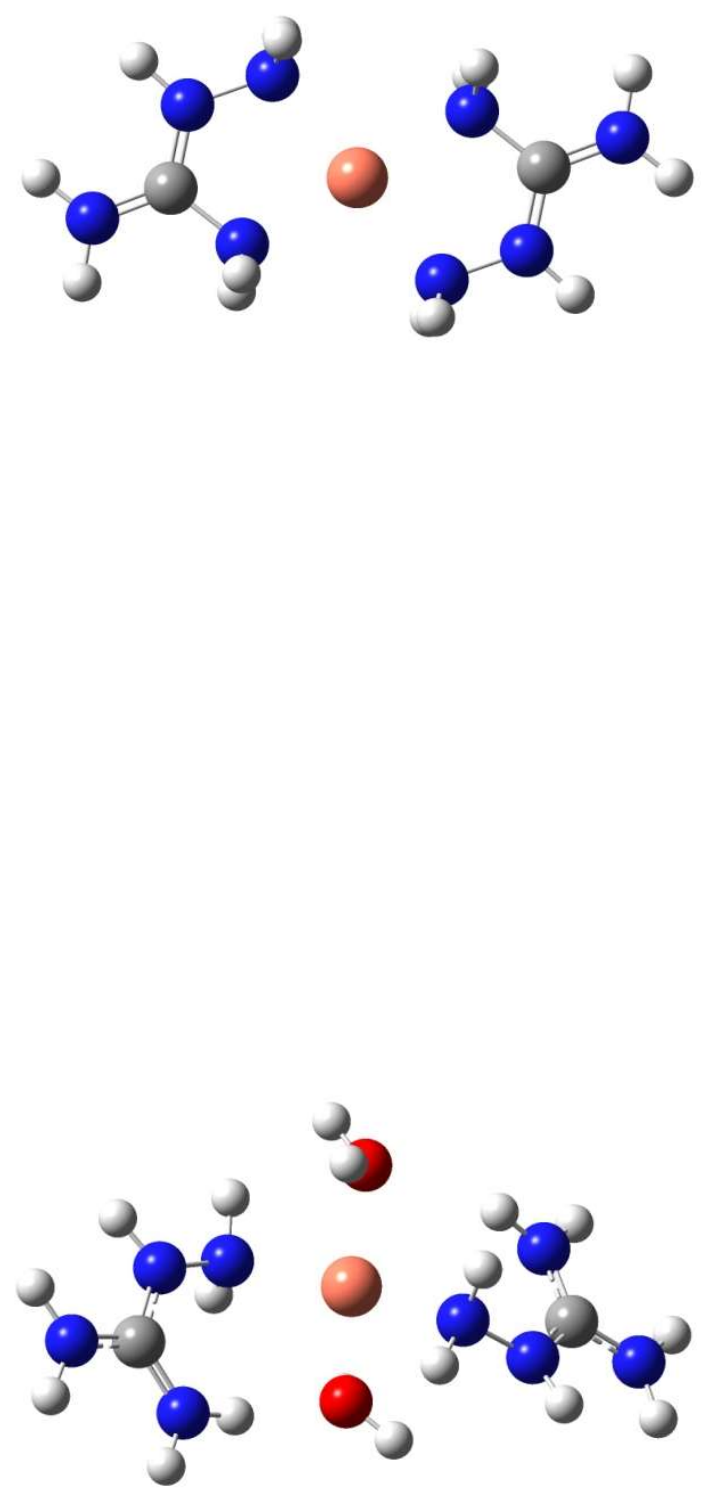

Charge $=4$ Multiplicity $=2$

$\mathrm{Cu}, 0,0.0388923171,-0.2283699935,0.3514127308$

$\mathrm{N}, 0,1.8830582865,-1.2580507379,0.1687666257$

$\mathrm{H}, 0,1.8374577093,-1.9621843017,0.9022415986$

$\mathrm{H}, \mathrm{0}, 1.9842485314,-1.7631646694,-0.7136196302$

$\mathrm{N}, 0,2.6441374632,0.9969773506,-1.313018926$

$\mathrm{H}, 0,2.9359154751,1.8204103411,-1.8160914299$

$\mathrm{C}, 0,3.38649641,0.5642404468,-0.3080200488$

$\mathrm{N}, 0,3.0289472551,-0.500521197,0.426981932$

$\mathrm{H}, 0,3.7037016695,-0.8996253333,1.0656981373$

$\mathrm{N}, 0,4.5144200678,1.1867670114,0.0157485226$

$\mathrm{H}, 0,4.856911012,1.936112536,-0.5646409666$

$\mathrm{H}, 0,5.111521925,0.8248562606,0.7419101686$

$\mathrm{N}, 0,-1.5840851378,1.112117741,0.5557000008$

$\mathrm{H}, 0,-1.8067042274,1.2545895593,1.5422637223$

$\mathrm{H}, 0,-1.1763915734,1.9808400594,0.2164538315$

$\mathrm{N}, 0,-2.7532309434,0.9174982025,-0.1809053164$

$\mathrm{C}, 0,-3.4562112289,-0.226262591,-0.1490372227$

$\mathrm{N}, 0,-3.0466544583,-1.271365147,0.5520248$

$\mathrm{N}, 0,-4.5712804415,-0.2856489913,-0.8645704789$

$\mathrm{H}, \mathrm{O},-5.1548279948,-1.1057255549,-0.8214929738$

$\mathrm{H}, \mathrm{0},-4.858608958,0.48942296,-1.4400933039$

$\mathrm{H}, \mathrm{O},-3.5958310377,-2.1167789844,0.5467059646$

$\mathrm{H}, \mathrm{O},-3.2061634945,1.7495546197,-0.5345046753$ 
$\mathrm{H}, \mathrm{0},-2.2947051631,-1.2013662378,1.2183573747$

$\mathrm{H}, \mathrm{O}, 1.7756369908,0.5531496807,-1.5844984107$

$0,0,-0.0379085627,-0.457167582,2.3913671151$

$\mathrm{H}, \mathrm{0}, 0.4632307793,-1.2051832126,2.7373045356$

$0,0,-0.0457003534,-0.2540534766,-1.7149227269$

$\mathrm{H}, 0,-0.7899611802,0.2104980538,-2.1170822309$

$\mathrm{H}, \mathrm{O}, 0.3255480405,0.3185595632,2.8362505444$

$\mathrm{H}, 0,-0.0992811771,-1.159522376,-2.0463362634$

\section{[3] $\left[\mathrm{Cu}(\mathrm{AGH})\left(\mathrm{H}_{2} \mathrm{O}\right)_{2}\right]^{3+}$}

Charge $=3$ Multiplicity $=2$

$\mathrm{Cu}, 0,-0.0292659945,-0.0888017861,0.0197236267$

$\mathrm{N}, 0,-1.3157718498,1.5227197806,0.0568588131$

$\mathrm{H}, 0,-1.1781986092,2.0959456525,0.8877343794$

$\mathrm{H}, 0,-1.1145658657,2.0941968764,-0.7626305163$

$\mathrm{N}, 0,-2.6414648622,1.0983130929,-0.0132163631$

C, $0,-2.9194185497,-0.1945251851,0.0656177592$

$\mathrm{N}, 0,-1.9018705282,-1.0127046566,0.5460073903$

$\mathrm{N}, 0,-4.0749326444,-0.676580993,-0.2960326882$

$\mathrm{H}, \mathrm{0},-4.3062085194,-1.6412342057,-0.1033736635$

$\mathrm{H}, \mathrm{O},-4.7745981017,-0.0844474603,-0.7240641932$

$\mathrm{H}, \mathrm{O},-2.0499587181,-1.9933696034,0.3219512947$

$\mathrm{H}, 0,-3.3466689417,1.7653596942,-0.3085322766$

$0,0,1.550688468,1.1836505668,-0.3331367744$

$\mathrm{H}, 0,2.3721092165,0.6781490803,-0.3663117293$

$0,0,1.0251275183,-1.8105472805,-0.1429060896$

$\mathrm{H}, 0,1.9677908623,-1.6376491878,-0.0315955176$

$\mathrm{H}, 0,1.6645269984,1.792755599,0.4066756483$

$\mathrm{H}, \mathrm{O}, 0.8004941128,-2.4395809525,0.5535277005$

$\mathrm{H}, \mathrm{O},-1.8191299918,-0.9104860316,1.5597871998$

\section{[4] $\left[\mathrm{Cu}(\mathrm{AGH})\left(\mathrm{H}_{2} \mathrm{O}\right)_{3}\right]^{3+}$}

Charge $=3$ Multiplicity $=2$

$\mathrm{Cu}, 0,-1.14335546,-0.1515644688,0.1024954719$ $\mathrm{N}, 0,0.560055998,0.7394893042,-0.7272568168$

$\mathrm{H}, 0,0.5671382048,0.6593703355,-1.7448664077$

$\mathrm{H}, \mathrm{O}, \mathrm{0} .4211228601,1.7288326817,-0.528980189$

$\mathrm{N}, 0,1.8210931774,0.4089385833,-0.2272649953$

$\mathrm{C}, 0,2.2302988802,-0.8631747197,-0.1168825659$

$\mathrm{N}, 0,1.5796473867,-1.841536429,-0.7266289482$

$\mathrm{N}, 0,3.3128581486,-1.1134967312,0.6107093803$

$\mathrm{H}, 0,3.7724610857,-2.0063201577,0.522547825$

$\mathrm{H}, 0,3.8345584023,-0.3514000437,1.0157519208$

$\mathrm{H}, 0,1.911247957,-2.7895576192,-0.6381193121$

$\mathrm{H}, \mathrm{O}, 2.2924663899,1.1264329509,0.3055313275$

$0,0,-2.9230819906,-0.8612181133,0.8126690326$

$\mathrm{H}, 0,-3.2233814035,-1.6098855666,0.2835742535$

$\mathrm{H}, \mathrm{O},-2.825147538,-1.2014245614,1.7099123931$

$\mathrm{H}, 0,0.7942044991,-1.666551892,-1.3329902957$

$0,0,-1.7810305797,-0.2994090954,-1.8795832951$

$\mathrm{H}, \mathrm{O},-2.6882093385,-0.6289946657,-1.88195212$

$0,0,-0.3736007164,0.2798495445,1.9720765784$

$\mathrm{H}, \mathrm{O},-1.0796081969,0.1946925061,2.6242987219$

$\mathrm{H}, \mathrm{O},-1.27142004,-0.9831907823,-2.3323121591$

$\mathrm{H}, 0,0.2803752736,-0.3830640601,2.2270302$
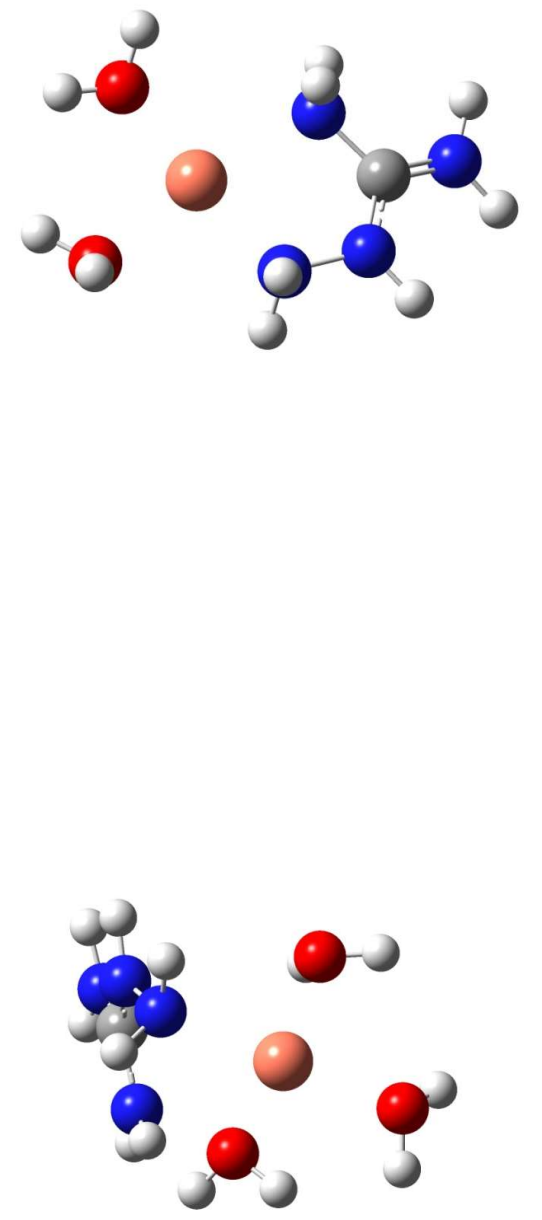


\section{[5] $\left[\mathrm{Cu}\left(\mathrm{AG}_{\mathrm{A}}\right)\left(\mathrm{H}_{2} \mathrm{O}\right)_{2}\right]^{2+}$}

Charge $=2$ Multiplicity $=2$

$\mathrm{N}, 0,1.7999026852,0.1618535382,-0.0333761331$

$\mathrm{H}, 0,2.3618305748,-0.3893051478,-0.673941742$

$\mathrm{H}, 0,2.3155859623,0.2495644414,0.8357869669$

$\mathrm{N}, 0,0.620796616,-0.5248118868,0.2217590928$

$\mathrm{C}, 0,-0.5484082285,0.0219619426,-0.0916827369$

$\mathrm{N}, 0,-0.8032948611,1.1692328219,-0.6241961741$

$\mathrm{N}, 0,-1.7501639415,-0.7010970205,0.1558117358$

$\mathrm{H}, 0,-1.8923501514,-0.8591781868,1.1503134066$

$\mathrm{H}, \mathrm{O},-1.7538677276,-1.6015834676,-0.3162653446$

$\mathrm{H}, 0,-0.0211155689,1.7713825645,-0.8523490987$

$\mathrm{H}, 0,0.6365900608,-1.4508586859,0.6319595192$

$\mathrm{Cu}, 0,-2.764690421,0.9972585626,-0.7524198486$

$0,0,-4.7535521893,0.5217128669,-0.6639719363$

$\mathrm{H}, \mathrm{O},-4.9026329378,-0.3863371377,-0.9516333625$

$\mathrm{H}, \mathrm{0},-5.2494288351,1.0688058728,-1.2850035459$

$0,0,-3.1075445933,2.7520333475,-1.840226685$

$\mathrm{H}, 0,-4.016622168,3.0524247416,-1.727579648$

$\mathrm{H}, 0,-2.5604879957,3.475402913,-1.5129220655$

\section{[6] $\left[\mathrm{Cu}\left(\mathrm{AG}_{\mathrm{A}}\right)\left(\mathrm{H}_{2} \mathrm{O}\right)_{3}\right]^{2+}$}

Charge $=2$ Multiplicity $=2$

$\mathrm{N}, 0,1.7312209096,-0.796234061,0.4096369418$

$\mathrm{H}, 0,2.4041836966,-1.1462163631,-0.2622256128$

$\mathrm{H}, 0,1.74551874,-1.40054614,1.223847783$

$\mathrm{N}, 0,0.4684026377,-0.8051527068,-0.1591547922$

$\mathrm{C}, 0,-0.2705594641,0.328941109,-0.1849550903$

$\mathrm{N}, 0,0.1900972824,1.463935542,0.2729856459$

$\mathrm{N}, 0,-1.5232758552,0.2125813251,-0.6804646474$

$\mathrm{H}, \mathrm{O},-2.000238789,1.0686722403,-0.9129736058$

$\mathrm{H}, \mathrm{O},-1.7302226725,-0.5803138719,-1.2671437747$

$\mathrm{H}, 0,1.1354948765,1.3538210908,0.6234642546$

$\mathrm{H}, \mathrm{O}, 0.0353350885,-1.6796845538,-0.422463621$

$\mathrm{Cu}, 0,-0.4901642869,3.2633200189,0.0058140495$

$0,0,-1.271342085,5.0269295179,-0.6346614898$

$\mathrm{H}, 0,-1.7287344249,5.4899557627,0.0771310776$

$\mathrm{H}, \mathrm{O},-0.587115738,5.6342882783,-0.9393362407$

$0,0,-2.2176478271,2.9844932259,1.1953388161$

$\mathrm{H}, \mathrm{O},-3.0200090877,3.1389175658,0.6836624324$

$\mathrm{H}, \mathrm{O},-2.2916052902,2.0755038907,1.5066678902$

$0,0,1.5076519032,3.9307662761,-0.3932196603$

$\mathrm{H}, 0,1.5559743695,4.8713218245,-0.1872522919$

$\mathrm{H}, 0,2.1466881666,3.5153094787,0.1963943057$
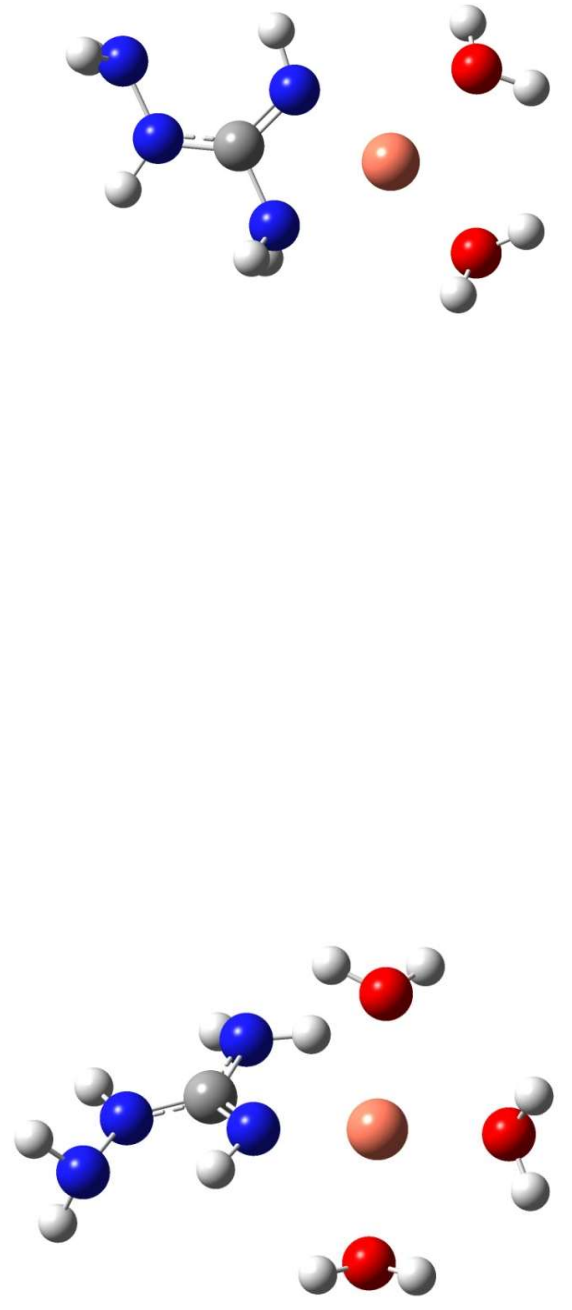


\title{
[7] $\left[\mathrm{Cu}\left(\mathrm{AG}_{\mathrm{B}}\right)\left(\mathrm{H}_{2} \mathrm{O}\right)_{2}\right]^{2+}$
}

Charge $=2$ Multiplicity $=2$

$\mathrm{Cu}, 0,-0.006073803,-0.0487636195,0.0157394263$

$\mathrm{N}, 0,-1.2957209627,1.5404248524,-0.0108560639$ $\mathrm{H}, \mathrm{O},-1.0718548981,2.1909926693,0.7371592694$ $\mathrm{H}, 0,-1.1641006075,2.0217114097,-0.8990308285$ $\mathrm{N}, 0,-2.6285327857,1.1145480493,0.1197627125$

$\mathrm{C}, 0,-2.8779371892,-0.2241900332,-0.0767653378$ $\mathrm{N}, 0,-3.9701292964,-0.6362029621,-0.5855712179$ $\mathrm{N}, 0,-1.7944603395,-1.0277310584,0.3998812672$ $\mathrm{H}, \mathrm{O},-1.8627839006,-1.9769517066,0.0465158887$ $\mathrm{H}, \mathrm{O},-1.8181892724,-1.0669919721,1.4187974008$ $\mathrm{H}, 0,-4.023693209,-1.6496238593,-0.5288232696$ $\mathrm{H}, \mathrm{O},-3.3062486434,1.7346944759,-0.3045861701$ $0,0,1.5839919562,1.2186800637,-0.3133641295$ $\mathrm{H}, \mathrm{O}, 2.4215739356,0.7430878687,-0.2674182304$ $0,0,1.1139320722,-1.7852512027,-0.0446975472$ $\mathrm{H}, 0,2.0409469598,-1.5811194057,0.1244957895$ $\mathrm{H}, 0,1.6512513006,1.9072650338,0.3586676075$ $\mathrm{H}, 0,0.863961593,-2.4051662434,0.6502843628$

\section{[8] $\left[\mathrm{Cu}\left(\mathrm{AG}_{\mathrm{B}}\right)\left(\mathrm{H}_{2} \mathrm{O}\right)_{3}\right]^{2+}$}

\author{
Charge $=2$ Multiplicity $=2$ \\ $\mathrm{N}, 0,-1.6265735153,-0.1674944528,-0.2438595007$ \\ $\mathrm{H}, 0,-2.2179531336,-0.8032806581,0.2797866998$ \\ $\mathrm{H}, 0,-2.016096075,-0.065726326,-1.173988399$ \\ $\mathrm{N}, 0,-0.3485319611,-0.6971808108,-0.3353865147$ \\ $\mathrm{C}, 0,0.7149461817,-0.0330405542,0.1608426198$ \\ $\mathrm{N}, 0,1.9080914162,-0.5904797617,0.1574589987$ \\ $\mathrm{N}, 0,0.5064574206,1.2254954066,0.5890249681$ \\ $\mathrm{H}, 0,1.1987231643,1.6448250078,1.187100025$ \\ $\mathrm{H}, \mathrm{O},-0.4539495163,1.516769183,0.6897420557$ \\ $\mathrm{H}, 0,2.6183682491,0.0459577832,0.4961637409$ \\ $\mathrm{H}, 0,-0.2013897989,-1.6183498738,-0.7203299389$ \\ $\mathrm{Cu}, 0,2.5897142106,-2.4103894352,0.0363970869$ \\ $0,0,4.5329981023,-1.5708774227,-0.2625877277$ \\ $\mathrm{H}, 0,5.1131601615,-2.2179175705,-0.680046762$ \\ $\mathrm{H}, 0,4.5107450908,-0.8271975398,-0.875255127$ \\ $0,0,0.8682487336,-3.6950258109,0.4178631174$ \\ $\mathrm{H}, 0,0.3585053765,-3.8717532895,-0.3805483809$ \\ $\mathrm{H}, 0,0.2448793793,-3.2771865524,1.0217685053$ \\ $0,0,3.481510413,-4.2531920497,-0.0412125249$ \\ $\mathrm{H}, \mathrm{O}, 4.3579957388,-4.2547328858,0.3604563873$ \\ $\mathrm{H}, 0,2.955921972,-4.8768906567,0.4727476509$
}

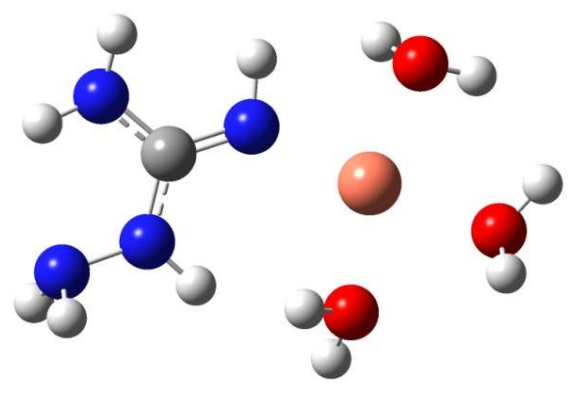




\section{[9] $\left[\mathrm{Cu}(\mathrm{AGc})\left(\mathrm{H}_{2} \mathrm{O}\right)_{2}\right]^{2+}$}

Charge $=2$ Multiplicity $=2$

$\mathrm{N}, 0,-1.7503812574,0.1903543528,-0.4328618793$

$\mathrm{H}, \mathrm{O},-2.4603006604,-0.1295304614,0.2232827258$

$\mathrm{H}, \mathrm{O},-2.1439112868,0.1431046632,-1.3689428974$

$\mathrm{N}, 0,-0.6164401514,-0.6367837473,-0.3468335346$

$\mathrm{C}, 0,0.5451253911,-0.0704484875,0.1225393013$

$\mathrm{N}, 0,1.5057763023,-0.6426911348,0.7329504041$

$\mathrm{N}, 0,0.6097409885,1.3170422627,-0.1971028776$

$\mathrm{H}, 0,0.7703701986,1.4393092736,-1.1962031123$

$\mathrm{H}, \mathrm{0}, 1.3740481619,1.7567715262,0.3043541736$

$\mathrm{H}, 0,1.2662540537,-1.6113493921,0.9361031971$

$\mathrm{H}, 0,-0.8057634618,-1.582693379,-0.0421051003$

$\mathrm{Cu}, 0,-1.2708212839,2.1269406365,0.0753713343$

$0,0,-3.2609596787,2.6504620072,0.1074879905$

$\mathrm{H}, \mathrm{O},-3.777454799,2.083688822,0.692742112$

$\mathrm{H}, 0,-3.3696158217,3.5414052491,0.4599381596$

$0,0,-0.5428871654,3.9975723533,0.585741549$

$\mathrm{H}, \mathrm{O},-0.0348502712,4.3747350828,-0.1420472574$

$\mathrm{H}, 0,-1.2710840586,4.6133945926,0.7286600816$

\section{$[10]\left[\mathrm{Cu}(\mathrm{AGc})\left(\mathrm{H}_{2} \mathrm{O}\right)_{3}\right]^{2+}$}

Charge $=2$ Multiplicity $=2$

$\mathrm{N}, 0,-1.6791346756,0.5587370522,0.088700744$

$\mathrm{H}, \mathrm{O},-2.3027902147,0.2602909995,0.8304459553$

$\mathrm{H}, 0,-2.2215755104,0.6689464495,-0.7600911423$

$\mathrm{N}, 0,-0.6995763084,-0.4009403423,-0.1135169359$

$\mathrm{C}, 0,0.6046436482,-0.1251910771,0.1258377019$

$\mathrm{N}, 0,1.5302616177,-1.0402318251,-0.0777736577$

$\mathrm{N}, 0,0.8965519651,1.0975186702,0.592748296$

$\mathrm{H}, 0,1.8542243797,1.4058190041,0.5077730629$

$\mathrm{H}, 0,0.1522288471,1.7765141469,0.554098818$

$\mathrm{H}, 0,1.1383745532,-1.9250852029,-0.3765009667$

$\mathrm{H}, \mathrm{O},-0.9561909276,-1.3499064884,-0.3472558376$

$\mathrm{Cu}, 0,3.4379552271,-1.093349761,0.3183805253$

$0,0,3.2730677,-3.1530396563,0.7051164804$

$\mathrm{H}, 0,4.1308350216,-3.5910018945,0.6676586998$

$\mathrm{H}, 0,2.7282629685,-3.6164791001,0.0587434253$

$0,0,3.7111416843,1.0150404127,-0.0326476905$

$\mathrm{H}, 0,4.499927397,1.3271548838,0.4258566665$

$\mathrm{H}, 0,3.8980490369,1.1481453203,-0.9694592015$

$0,0,5.4844271098,-1.1530043664,0.6493682576$

$\mathrm{H}, 0,5.933423708,-0.5659897573,0.0303759777$

$\mathrm{H}, \mathrm{O}, 5.8537440327,-2.0274819077,0.4812953216$
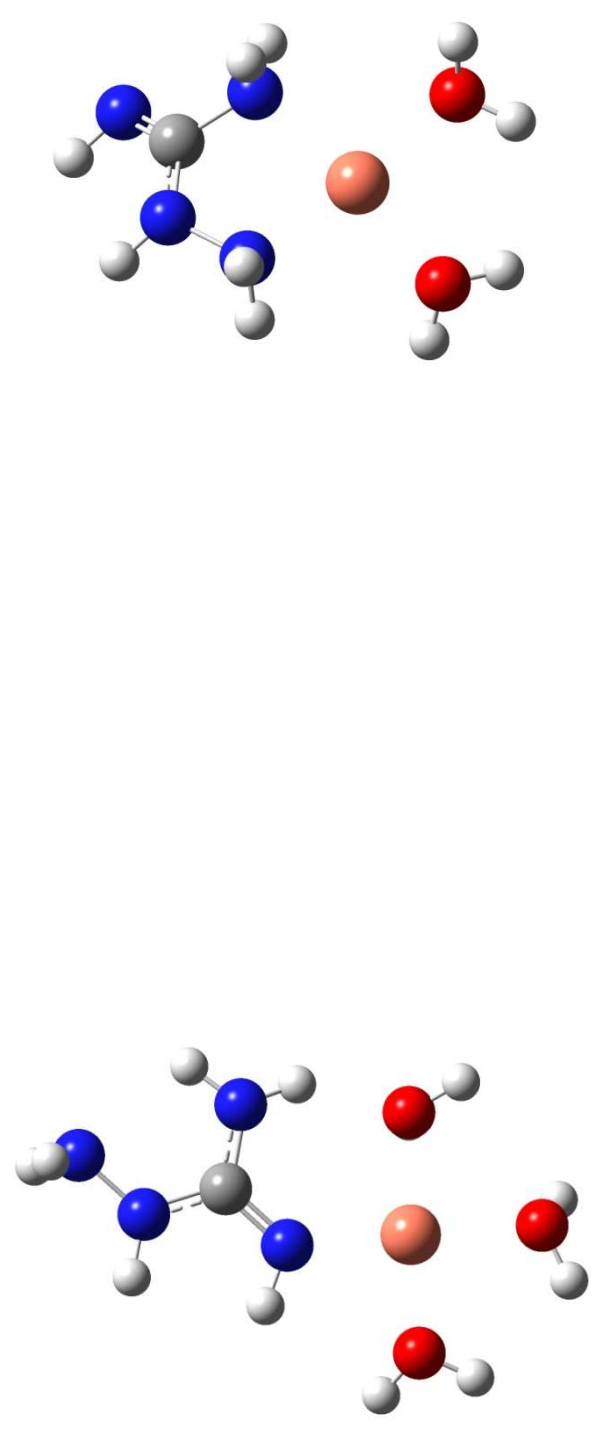


\section{$[11]\left[\mathrm{Cu}\left(\mathrm{AG}_{\mathrm{D}}\right)\left(\mathrm{H}_{2} \mathrm{O}\right)_{2}\right]^{2+}$}

Charge $=2$ Multiplicity $=2$

$\mathrm{Cu}, 0,-0.0765043509,-0.0822187124,0.0281434103$

$\mathrm{N}, 0,-1.3397469494,1.5618825734,-0.1156145179$

$\mathrm{H}, \mathrm{O},-1.1100902063,2.2637948285,0.5823921462$

$\mathrm{H}, 0,-1.2235279066,1.9770470965,-1.0377663313$

$\mathrm{N}, 0,-2.6580381896,1.1353020616,0.0669834544$

C, $0,-2.8524277245,-0.2160678576,0.046732905$

$\mathrm{N}, 0,-1.7973507469,-0.9791222587,0.1158138161$

$\mathrm{N}, 0,-4.1189417698,-0.648601477,-0.0418360141$

$\mathrm{H}, \mathrm{O},-4.3007582366,-1.6193748861,0.1569202714$

$\mathrm{H}, \mathrm{0},-4.8740826428,-0.0010235899,0.1215322204$

$\mathrm{H}, 0,-1.9804488272,-1.9726298867,0.1481129094$

$\mathrm{H}, \mathrm{O},-3.3792186082,1.732170843,-0.3157548204$

$0,0,1.5743616774,1.1333859155,-0.2573507308$

$\mathrm{H}, \mathrm{O}, 2.3901232677,0.6360809149,-0.1264564538$

$0,0,1.0190032645,-1.8279536976,0.1635835255$

$\mathrm{H}, 0,1.9344019696,-1.6407389013,0.4002166722$

$\mathrm{H}, 0,1.6149615419,1.8509068902,0.3859062593$

$\mathrm{H}, 0,0.6905924376,-2.4048848564,0.8626002781$

\section{$[12]\left[\mathrm{Cu}\left(\mathrm{AG}_{\mathrm{A}}\right)_{2}\right]^{2+}$}

Charge $=2$ Multiplicity $=2$

$\mathrm{N}, 0,-4.6382895129,-0.6947170809,0.1025582332$

$\mathrm{H}, \mathrm{O},-5.2931639594,-0.6462366202,-0.6705553806$

$\mathrm{H}, 0,-5.171729535,-0.6618524656,0.9651355694$

$\mathrm{N}, 0,-3.8098542731,0.4182792692,0.051294022$

$C, 0,-2.4910452722,0.2611305328,-0.0091402126$

$\mathrm{N}, 0,-1.7975272442,-0.8264832196,-0.0400772638$

$\mathrm{N}, 0,-1.6484423959,1.4107982321,-0.041238959$

$\mathrm{H}, \mathrm{O},-1.8048981242,2.0172378943,0.7596053087$

$\mathrm{H}, 0,-1.7959803775,1.9542560053,-0.8881864552$

$\mathrm{H}, \mathrm{O},-2.3059077981,-1.7024075296,-0.0161374644$

$\mathrm{H}, \mathrm{O},-4.1953638028,1.3545089266,0.0741703176$

$\mathrm{N}, 0,4.6350858353,0.689646972,0.1072444255$

$\mathrm{H}, \mathrm{O}, 5.2909295628,0.6456619101,-0.6652968773$

$\mathrm{H}, 0,5.1674157533,0.652236738,0.970331527$

$\mathrm{N}, 0,3.8070711892,-0.4233186481,0.0487479001$

$\mathrm{C}, 0,2.4881788607,-0.266234672,-0.0097804444$

$\mathrm{N}, 0,1.7942892749,0.8213178442,-0.03347054$

$\mathrm{N}, 0,1.645891371,-1.416001044,-0.0487770694$

$\mathrm{H}, 0,1.8010552901,-2.0256451233,0.7498988452$

$\mathrm{H}, 0,1.79546533,-1.9560164148,-0.8975535617$

$\mathrm{H}, \mathrm{O}, 2.3024463478,1.6972083919,-0.0039228787$

$\mathrm{H}, \mathrm{O}, 4.1928210307,-1.3595551815,0.0670146431$

$\mathrm{Cu}, 0,-0.0014925507,-0.002828717,-0.0409146845$

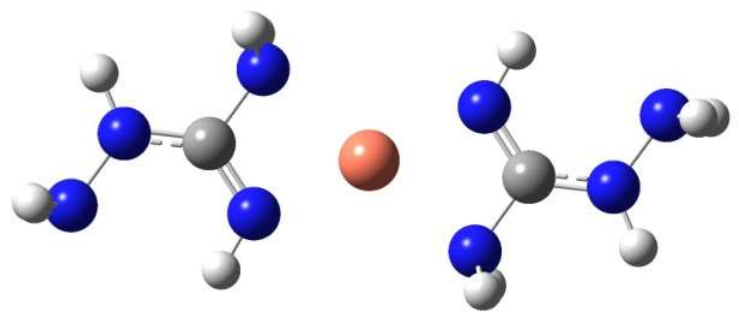




\section{$[13]\left[\mathrm{Cu}\left(\mathrm{AG}_{\mathrm{A}}\right)_{2}\right]^{2+}$ (mirror image)}

Charge $=2$ Multiplicity $=2$ $\mathrm{N}, 0,-4.6213205954,-0.7129254901,0.0910204988$ $\mathrm{H}, \mathrm{O},-5.28380683,-0.6360106644,-0.6730447061$ $\mathrm{H}, \mathrm{O},-5.1455793808,-0.722256375,0.959793715$ $\mathrm{N}, 0,-3.7981419693,0.4050399432,0.078745673$ $\mathrm{C}, 0,-2.4791910798,0.2512194329,0.0137037011$ $\mathrm{N}, 0,-1.778150621,-0.8279984437,-0.0506649257$ $\mathrm{N}, 0,-1.6380005128,1.4061935272,0.0139874761$ $\mathrm{H}, \mathrm{O},-1.7798795045,1.9751820982,0.8450391999$ $\mathrm{H}, \mathrm{O},-1.8057247352,1.9896375272,-0.8023019087$ $\mathrm{H}, 0,-2.2799520585,-1.7080451683,-0.0529804349$ $\mathrm{H}, \mathrm{O},-4.1850124751,1.338854419,0.1448868347$ $\mathrm{N}, 0,4.4480929915,-1.442999354,0.0099047615$ $\mathrm{H}, 0,4.98028753,-1.5423184848,0.8681340692$ $\mathrm{H}, 0,5.0998620601,-1.4677321089,-0.76677693$ $\mathrm{N}, 0,3.8144338864,-0.2076598306,0.0182402644$ $\mathrm{C}, 0,2.4871067955,-0.1477692922,-0.0278601499$ $\mathrm{N}, 0,1.6214347035,-1.1003438502,-0.0869650367$ $\mathrm{N}, 0,1.841847637,1.1268151632,-0.0090806676$ $\mathrm{H}, 0,2.0856594694,1.6792678499,-0.8277863386$ $\mathrm{H}, 0,2.0883475201,1.6623393566,0.8197153437$ $\mathrm{H}, 0,1.9755341186,-2.0493794767,-0.1014389362$ $\mathrm{H}, 0,4.34712635,0.6518384654,0.0777539542$ $\mathrm{Cu}, 0,0.0057507105,0.082006746,-0.0466214571$

\section{$[14]\left[\mathrm{Cu}\left(\mathrm{AG}_{\mathrm{A}}\right)_{2}\left(\mathrm{H}_{2} \mathrm{O}\right)_{2}\right]^{2+}$}

Charge $=2$ Multiplicity $=2$

$\mathrm{N}, 0,4.5196231342,-0.9100210412,-0.8112989238$ $\mathrm{H}, 0,5.0248637743,-1.7216015206,-0.4770500419$ $\mathrm{H}, \mathrm{O}, 5.1265607564,-0.3936528084,-1.4392045006$ $\mathrm{N}, 0,4.1755005645,-0.1142650473,0.2711024115$ C, $0,2.8980272523,0.3279827274,0.398558757$ $\mathrm{N}, 0,1.9414816669,-0.071167831,-0.3980799275$ $\mathrm{N}, 0,2.6860535408,1.2246208895,1.3875174969$ $\mathrm{H}, 0,3.3639895557,1.2834952004,2.1306111868$ $\mathrm{H}, 0,1.7298806937,1.4135514356,1.6395795172$ $\mathrm{H}, 0,2.308345787,-0.7202756237,-1.0863177886$ $\mathrm{H}, \mathrm{O}, 4.9017416953,0.3706584875,0.7809996625$ $\mathrm{N}, 0,-4.5554810432,0.8986285103,-0.6849905692$ $\mathrm{H}, 0,-5.1855891991,1.5742556645,-0.2704578501$ $\mathrm{H}, \mathrm{O},-5.0348554135,0.4457226972,-1.4560675579$ $\mathrm{N}, 0,-4.2066529097,-0.0429047319,0.2717692925$ C, $0,-2.8993979162,-0.3739808389,0.436994968$ $\mathrm{N}, 0,-1.9394713274,0.2903840857,-0.148559054$ $\mathrm{N}, 0,-2.6589756251,-1.4359885201,1.2383198508$ $\mathrm{H}, \mathrm{O},-3.3955804708,-1.737284006,1.8566903007$ $\mathrm{H}, 0,-1.7200999422,-1.5409569965,1.5865216446$ $\mathrm{H}, \mathrm{0},-2.3310561565,1.0196831831,-0.7361041673$ $\mathrm{H}, \mathrm{O},-4.9080420794,-0.6889395157,0.608044989$ $\mathrm{Cu}, 0,0.0034216282,0.0718384591,-0.2046649559$ $0,0,-0.0628909677,-2.1326825205,-0.3223801272$ $\mathrm{H}, 0,-0.8707628113,-2.4482801702,-0.7423042148$ $\mathrm{H}, 0,0.6505835233,-2.4673214179,-0.8766371409$ $0,0,0.1442494095,2.2668901959,-0.1144382457$ $\mathrm{H}, \mathrm{O},-0.6466878934,2.7042765974,-0.4475641584$ $\mathrm{H}, 0,0.8593437833,2.5802134662,-0.6792858637$
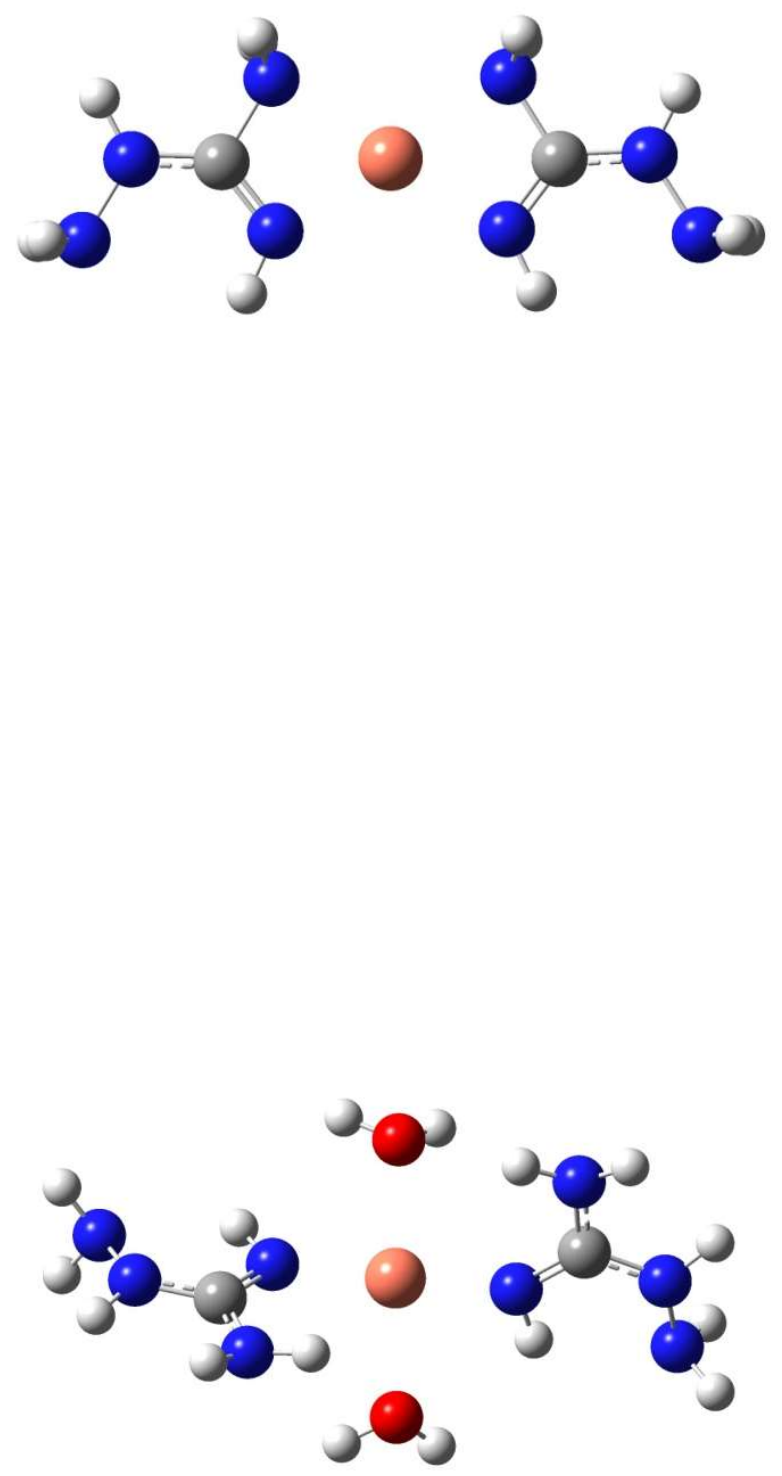


\section{$[15]\left[\mathrm{Cu}\left(\mathrm{AG}_{\mathrm{B}}\right)_{2}\right]^{2+}$}

Charge $=2$ Multiplicity $=2$

$\mathrm{Cu}, 0,0.0152975233,-0.00257552,0.1466317136$

$\mathrm{N}, 0,1.3803233946,-1.5289987677,0.3778940336$

$\mathrm{H}, 0,1.200721766,-2.0512667269,1.2308106013$

$\mathrm{H}, 0,1.2832777567,-2.1683782555,-0.4088204412$

$\mathrm{N}, 0,3.9216359413,0.7002146446,-0.5407864014$

$\mathrm{H}, 0,3.9190108716,1.7135090758,-0.6199826602$ $\mathrm{C}, 0,2.8583433141,0.3014553929,0.0364327187$

$\mathrm{N}, 0,2.6873431597,-1.0099670161,0.4140608975$

$\mathrm{H}, 0,3.3986711935,-1.6421769419,0.0712416882$

$\mathrm{N}, 0,-1.3508760125,1.5221970056,-0.0846007032$ $\mathrm{H}, \mathrm{0},-1.254878288,2.1613647852,0.7024223194$

$\mathrm{H}, \mathrm{O},-1.1714378266,2.0448876996,-0.9372822478$

$\mathrm{N}, 0,-2.6571640475,1.0014839215,-0.1218400103$

$\mathrm{C}, 0,-2.8273270042,-0.3094612932,0.2580799561$ $\mathrm{N}, 0,-3.8907434636,-0.708020726,0.8351515814$

$\mathrm{H}, 0,-3.8874829206,-1.7211781023,0.9160774566$ $\mathrm{H}, \mathrm{0},-3.3696990291,1.6334808403,0.218893511$ $\mathrm{N}, 0,-1.7031534345,-1.1110736716,-0.116436806$ $\mathrm{H}, 0,-1.7361055648,-2.0145394746,0.3456668105$ $\mathrm{H}, 0,-1.7161501663,-1.2720005674,-1.1234988841$ $\mathrm{N}, 0,1.7351242163,1.1032154039,0.4130307503$ $\mathrm{H}, 0,1.7474627519,1.2604412672,1.4206793941$ $\mathrm{H}, \mathrm{O}, 1.7694998685,2.0082860265,-0.045788278$

\section{$[16]\left[\mathrm{Cu}(\mathrm{AG})_{2}\right]^{2+}$ (mirror image)}

Charge $=2$ Multiplicity $=2$

$\mathrm{Cu}, 0,0.0250755414,0.1884774014,0.1981617717$ $\mathrm{N}, 0,-1.3822294124,1.680115207,0.0852376434$ $\mathrm{H}, \mathrm{O},-1.2573767966,2.3550614699,0.8344057031$ $\mathrm{H}, \mathrm{0},-1.2577351403,2.1705531868,-0.7990388876$ $\mathrm{N}, 0,-3.8025982842,-0.7419038119,-0.601616091$ $\mathrm{H}, \mathrm{O},-3.7700479404,-1.7546105107,-0.5218611324$ C, $0,-2.7915940522,-0.221754962,-0.0278189357$ $\mathrm{N}, 0,-2.6785281971,1.1378452499,0.1534590073$ $\mathrm{H}, \mathrm{O},-3.3832265385,1.6833515146,-0.3260247126$ $\mathrm{N}, 0,1.6936656992,1.3524213551,-0.0807782033$ $\mathrm{H}, 0,1.6042610515,1.8461349203,-0.9674700648$ $\mathrm{H}, 0,1.7680789907,2.0476129884,0.6565937955$ $\mathrm{N}, 0,2.851299099,0.5551508298,-0.0881200367$ C, $0,2.6776947178,-0.7963188799,-0.2731477462$ $\mathrm{N}, 0,3.5370860691,-1.5068356858,-0.8884657477$ $\mathrm{H}, 0,3.3019663881,-2.4921555617,-0.8043602384$ $\mathrm{H}, \mathrm{O}, 3.6305680371,0.9529772091,-0.5966020614$ $\mathrm{N}, 0,1.4703133493,-1.2533544152,0.3480642934$ $\mathrm{H}, 0,1.2273229183,-2.1820668077,0.0166986019$ $\mathrm{H}, \mathrm{O}, 1.6197456969,-1.3160644383,1.3559723947$ $\mathrm{N}, 0,-1.6734799518,-0.9127194581,0.5383681486$ $\mathrm{H}, \mathrm{O},-1.7485482486,-0.9012332733,1.5559831286$ $\mathrm{H}, \mathrm{O},-1.6605180064,-1.8824485379,0.2378513695$
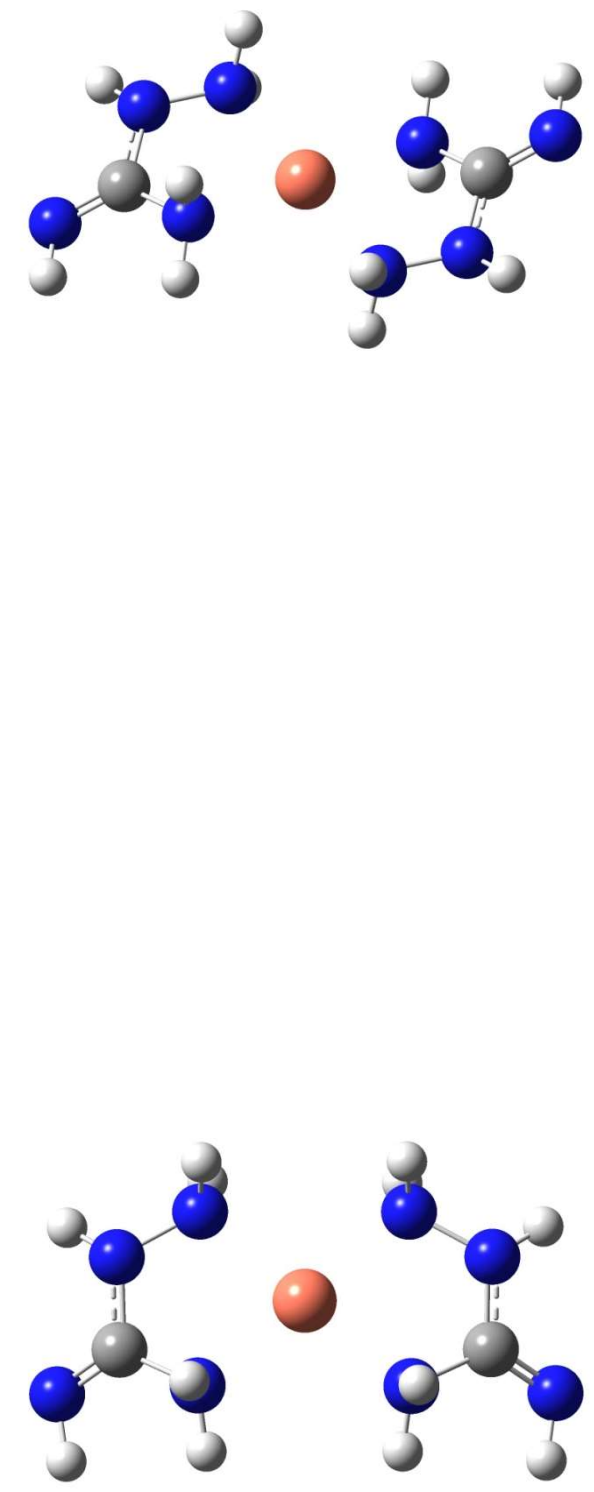


\section{$[17]\left[\mathrm{Cu}(\mathrm{AG})_{2}\left(\mathrm{H}_{2} \mathrm{O}\right)\right]^{2+}$}

Charge $=2$ Multiplicity $=2$

$\mathrm{Cu}, 0,0.074395468,0.1381630104,0.9861004566$ $\mathrm{N}, 0,-1.3099734778,1.6036551669,0.416642823$ $\mathrm{H}, \mathrm{O},-1.5769798878,2.1778705708,1.2106924415$ $\mathrm{H}, \mathrm{O},-0.8568901095,2.2030889493,-0.2696978929$ $\mathrm{N}, 0,-2.9879789617,-0.8095798171,-1.4775788317$ $\mathrm{H}, \mathrm{O},-2.8985832909,-1.8222641979,-1.4852401522$ C, $0,-2.3575415813,-0.3237477128,-0.4824875606$ $\mathrm{N}, 0,-2.4533737461,1.0060952541,-0.142124163$ $\mathrm{H}, \mathrm{O},-2.9329818082,1.5771633541,-0.8260680905$ $\mathrm{N}, 0,4.5452563318,0.4157881163,-0.177563324$ $\mathrm{H}, 0,4.663348754,0.2947618513,-1.1761149453$ $\mathrm{H}, \mathrm{O}, 4.9438045339,-0.3927758266,0.2878975868$ $\mathrm{N}, 0,3.1875297303,0.5019266761,0.1091778417$ C, $0,2.7292874777,1.4872514142,0.9231544073$ $\mathrm{N}, 0,1.5007279728,1.4343302912,1.3830756522$ $\mathrm{H}, 0,1.2401567528,2.2719832966,1.8875401569$ $\mathrm{H}, 0,2.6174548857,-0.3301511075,0.039606901$ $\mathrm{N}, 0,3.5637699546,2.5152164565,1.1614102295$ $\mathrm{H}, 0,3.3344470213,3.1537127526,1.9044571822$ $\mathrm{H}, 0,4.5360221527,2.3725308621,0.9328055816$ $\mathrm{N}, 0,-1.5196244695,-1.0344259858,0.4304605279$ $\mathrm{H}, \mathrm{O},-2.010147107,-1.1610127863,1.3157767466$ $\mathrm{H}, \mathrm{0},-1.2881820858,-1.9543423049,0.0690842252$ $0,0,1.0378304635,-1.5852704153,1.690435671$ $\mathrm{H}, 0,1.881286153,-1.3849059042,2.1127541817$ $\mathrm{H}, 0,1.2591866333,-2.1716784343,0.9572385875$

\section{$[18]\left[\mathrm{Cu}(\mathrm{AGB})_{2}\left(\mathrm{H}_{2} \mathrm{O}\right)_{2}\right]^{2+}$}

Charge $=2$ Multiplicity $=2$

$\mathrm{Cu}, 0,0.0870945982,-0.0903805241,0.4840203444$ $\mathrm{N}, 0,-4.5363330232,-0.3102307855,-0.3696136681$ $\mathrm{H}, \mathrm{O},-4.7348546705,0.2559240584,-1.187956103$ $\mathrm{H}, \mathrm{O},-5.0075201112,0.1106356563,0.4221907658$ $\mathrm{N}, 0,-1.1313777694,-1.399623733,-0.2979007636$ $\mathrm{H}, \mathrm{O},-0.7322333598,-2.3265205628,-0.3747753368$ C, $0,-2.447570402,-1.4432971574,-0.3318555562$ $\mathrm{N}, 0,-3.1658285544,-0.3137978272,-0.1267382758$ $\mathrm{H}, 0,-2.6500515761,0.5580146464,-0.1528641983$ $\mathrm{N}, 0,4.5839320725,0.6549804571,-0.0212560228$ $\mathrm{H}, 0,4.8200960021,0.7376629057,-1.0027649899$ $\mathrm{H}, 0,4.9851543481,-0.2092897934,0.327757889$ $\mathrm{N}, 0,3.2006560511,0.6141129274,0.1079507777$ C, 0,2.5767543259,1.4055760463,1.0179471872 $\mathrm{N}, 0,1.3116193347,1.1963226496,1.2989830265$ $\mathrm{H}, 0,0.9243345337,1.9098511362,1.9027716557$ $\mathrm{H}, 0,2.7035651899,-0.2160051103,-0.1856447734$ $\mathrm{N}, 0,3.2953404643,2.4175667059,1.5388447712$ $\mathrm{H}, 0,2.9356930979,2.8798127534,2.3571050257$ $\mathrm{H}, \mathrm{O}, 4.2956969686,2.3711588787,1.4162254277$ $\mathrm{N}, 0,-3.1370760086,-2.5804765197,-0.5228176692$ $\mathrm{H}, \mathrm{0},-4.1218032795,-2.4987844256,-0.722122715$ $\mathrm{H}, \mathrm{O},-2.6447484631,-3.407086216,-0.8156148489$ $0,0,1.1563999097,-1.7518330958,1.4025727492$ $\mathrm{H}, 0,1.8458451468,-1.3952385813,1.9739666482$ $\mathrm{H}, 0,1.6324147091,-2.2393290687,0.7208152578$ $0,0,-0.9776635442,1.5906293406,-0.4637843679$ $\mathrm{H}, \mathrm{O},-0.6104532995,2.4194431662,-0.1366940603$ $\mathrm{H}, 0,-0.7767007111,1.5850464224,-1.406935647$
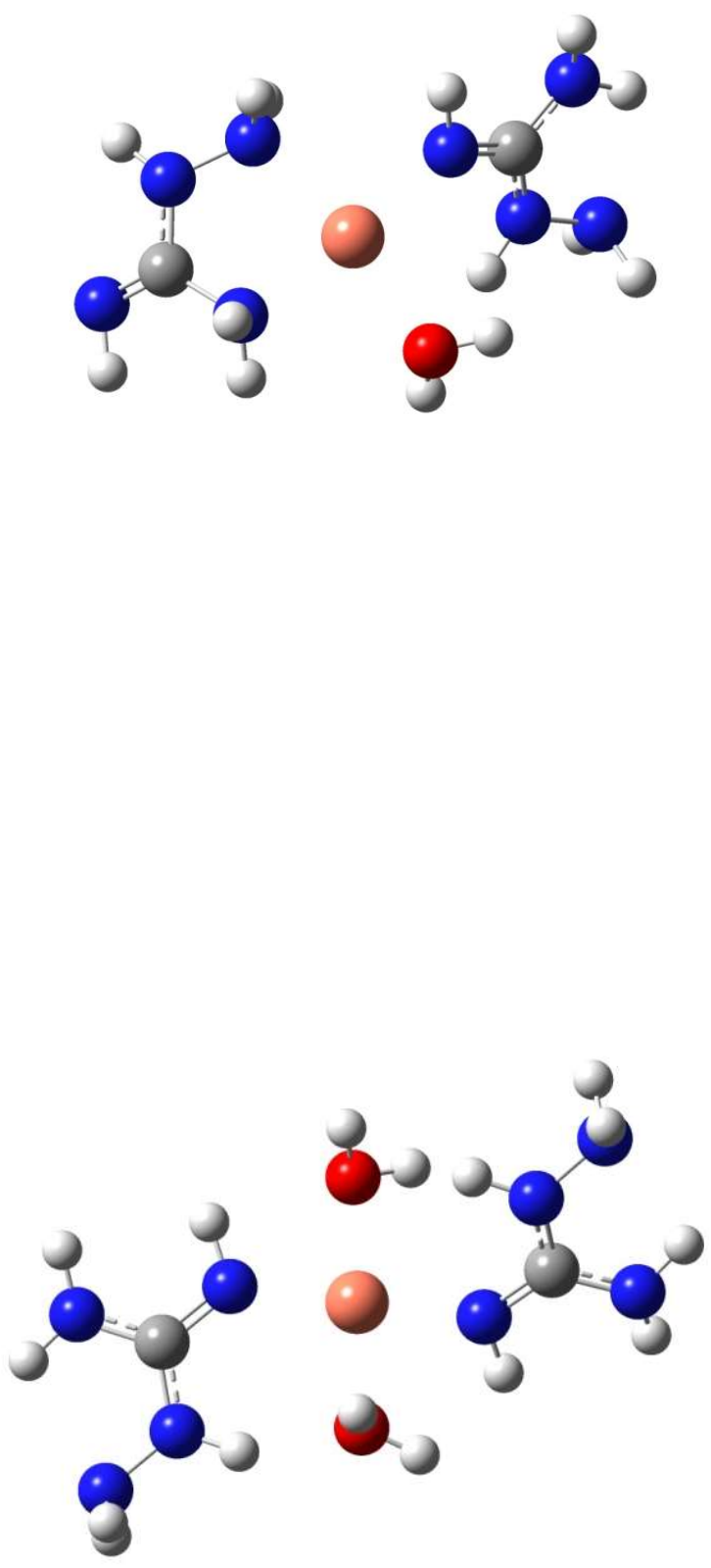


\section{$[19]\left[\mathrm{Cu}(\mathrm{AGc})_{2}\right]^{2+}$}

Charge $=2$ Multiplicity $=2$

$\mathrm{N}, 0,-1.2854004808,1.5945781308,-0.6456790762$ $\mathrm{H}, 0,-0.9663247053,2.3873567223,-0.0917435423$ $\mathrm{H}, \mathrm{O},-1.2528829494,1.8612213988,-1.6259681886$ $\mathrm{N}, 0,-2.6055693587,1.2634048749,-0.292448756$ $\mathrm{C}, 0,-2.8068577324,0.0988241465,0.4103612436$ $\mathrm{N}, 0,-3.7361962755,-0.162588185,1.241528861$ $\mathrm{N}, 0,-1.8432711156,-0.896833833,0.0622517098$ $\mathrm{H}, 0,-2.0699748287,-1.282345418,-0.854604526$ $\mathrm{H}, \mathrm{O},-4.29098689,0.6724395834,1.4219831863$ $\mathrm{H}, \mathrm{0},-3.1765907394,2.050626635,-0.0130311783$ $\mathrm{N}, 0,1.2154483792,-1.5930397454,0.1333745173$ $\mathrm{H}, 0,0.9692672831,-1.9705862655,1.0466237056$ $\mathrm{H}, 0,1.1002134259,-2.3319617319,-0.5549904442$ $\mathrm{N}, 0,2.5538287739,-1.1613264509,0.1327364429$ $\mathrm{C}, 0,2.7892300486,0.1931340275,0.0994213031$ $\mathrm{N}, 0,3.7855912251,0.8261811408,0.5783516426$ $\mathrm{N}, 0,1.7706358808,0.8841683664,-0.6252941921$ $\mathrm{H}, 0,1.9002132968,0.7264410051,-1.6247604456$ $\mathrm{H}, 0,1.8493749404,1.8819706137,-0.4572878627$ $\mathrm{H}, 0,4.3773170888,0.1947757566,1.1157783076$ $\mathrm{H}, 0,3.1704066629,-1.7011509843,0.7262581446$ $\mathrm{Cu}, 0,-0.0349372244,0.0007696116,-0.2561946814$ $\mathrm{H}, \mathrm{O},-1.8801659954,-1.6565602793,0.7341989191$

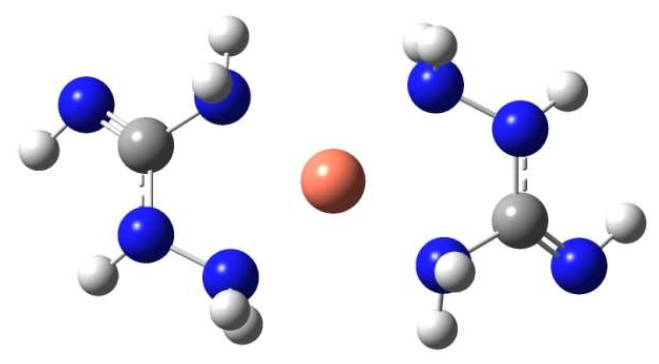

$[20]\left[\mathrm{Cu}(\mathrm{AGc})_{2}\right]^{2+}$ (mirror image)

Charge $=2$ Multiplicity $=2$

$\mathrm{N}, 0,1.6343184415,1.2386463129,-0.701073024$

$\mathrm{H}, 0,1.4072015768,1.8868265191,-1.4527600232$

$\mathrm{H}, 0,1.8843447279,1.7831115437,0.1198637739$

$\mathrm{N}, 0,2.719904682,0.4295040047,-1.0779712757$

$\mathrm{C}, 0,2.4722813082,-0.8990016483,-1.3270051265$

$\mathrm{N}, 0,3.1056277764,-1.6794983149,-2.109866194$

$\mathrm{N}, 0,1.3941737007,-1.3927653587,-0.5304272247$ $\mathrm{H}, 0,1.7139969547,-1.5273297001,0.4287890974$

$\mathrm{H}, \mathrm{O}, 1.1011345949,-2.2975662782,-0.8846439385$ $\mathrm{H}, 0,3.797271742,-1.1558403089,-2.6429495551$ $\mathrm{H}, 0,3.3887207638,0.8765704545,-1.6920352229$ $\mathrm{N}, 0,-1.4321453553,1.5595088803,-0.2999061994$ $\mathrm{H}, 0,-1.1895845028,2.1901561775,0.4624376609$ $\mathrm{H}, 0,-1.419594841,2.0951946303,-1.1636779362$ $\mathrm{N}, 0,-2.7193605342,1.0332447454,-0.0910039524$ $\mathrm{C}, 0,-2.8163565874,-0.2905760146,0.268971521$ $\mathrm{N}, 0,-3.7277193004,-0.845472589,0.9649688741$ $\mathrm{N}, 0,-1.7589877563,-1.0671743019,-0.2936201387$ $\mathrm{H}, \mathrm{O},-1.9409882599,-1.2173400707,-1.2862498687$ $\mathrm{H}, \mathrm{O},-1.7268881183,-1.9773113425,0.1541215372$ $\mathrm{H}, \mathrm{O},-4.3630325487,-0.1413415206,1.3364355838$ $\mathrm{H}, \mathrm{O},-3.3591741993,1.6642857655,0.3746995947$ $\mathrm{Cu}, 0,-0.0400821353,0.0499915542,-0.3504454829$






\section{$[21]\left[\mathrm{Cu}(\mathrm{AGc})_{2}\left(\mathrm{H}_{2} \mathrm{O}\right)\right]^{2+}$}

Charge $=2$ Multiplicity $=2$

$\mathrm{N}, 0,4.5346212943,-0.9909799152,0.432357055$

$\mathrm{H}, 0,5.2003774274,-0.4247336308,0.946749675$

$\mathrm{H}, 0,5.0408855301,-1.5164732533,-0.270980371$

$\mathrm{N}, 0,3.6068589583,-0.1670697541,-0.1875095365$

$\mathrm{C}, 0,2.2923667129,-0.2325617753,0.1321632835$

$\mathrm{N}, 0,1.4362373157,0.6411304229,-0.3427953075$ $\mathrm{N}, 0,1.9056183322,-1.2582423753,0.9146465164$ $\mathrm{H}, 0,2.6461908629,-1.7567111713,1.384823052$ $\mathrm{H}, 0,1.0263956107,-1.1659910364,1.3953304954$ $\mathrm{H}, 0,1.8836708186,1.3714147735,-0.8831555411$ $\mathrm{H}, 0,3.9165036545,0.5917643447,-0.77876768$ $\mathrm{N}, 0,-2.5586535459,0.9113331951,-0.1439332018$ $\mathrm{H}, \mathrm{O},-2.8028328529,1.3380876287,0.7473650869$ $\mathrm{H}, \mathrm{0},-2.8998198273,1.5185523618,-0.8837517249$ $\mathrm{N}, 0,-3.1632415671,-0.3536892171,-0.2513538985$ $\mathrm{C}, 0,-2.3410048783,-1.4539512359,-0.1649938504$ $\mathrm{N}, 0,-2.6343091439,-2.6122136981,0.280002669$ $\mathrm{N}, 0,-1.0511017957,-1.1852270456,-0.6999515533$ $\mathrm{H}, 0,-1.1059697715,-1.1140545592,-1.715231726$ $\mathrm{H}, 0,-0.4151818814,-1.9410480522,-0.4703145635$ $\mathrm{H}, \mathrm{O},-3.568748343,-2.6026068554,0.685292035$ $\mathrm{H}, \mathrm{O},-4.0547227762,-0.4354210374,0.220245813$ $\mathrm{Cu}, 0,-0.503585451,0.7787218372,-0.2029232917$ $0,0,-0.4702061315,2.8176254266,0.3288827815$ $\mathrm{H}, \mathrm{0},-0.0805569266,2.8929006559,1.2077274836$ $\mathrm{H}, 0,0.1363453948,3.2980499659,-0.2464316902$

\section{$[22]\left[\mathrm{Cu}(\mathrm{AGC})_{2}\left(\mathrm{H}_{2} \mathrm{O}\right)_{2}\right]^{2+}$}

Charge $=2$ Multiplicity $=2$

$\mathrm{N}, 0,3.7160212843,-3.6814829256,-0.3923431039$ $\mathrm{H}, 0,4.6005634322,-3.3403696699,-0.0316497779$ $\mathrm{H}, 0,3.9065050319,-4.4182589494,-1.0606982174$ $\mathrm{N}, 0,3.0392197851,-2.6522081921,-1.030139541$ $\mathrm{C}, 0,1.8931659006,-2.1472837858,-0.5115404298$ $\mathrm{N}, 0,1.2793197839,-1.1318929121,-1.073745711$ $\mathrm{N}, 0,1.4286094292,-2.7176584823,0.6155424383$ $\mathrm{H}, 0,1.8437094546,-3.5986301034,0.8771238292$ $\mathrm{H}, \mathrm{O}, 0.4627157698,-2.561326161,0.8513961552$ $\mathrm{H}, 0,1.7780773932,-0.7565816091,-1.8706515826$ $\mathrm{H}, 0,3.4934772352,-2.1292903443,-1.7659842901$ $\mathrm{N}, 0,-3.7094322949,3.5324453775,1.5656982467$ $\mathrm{H}, \mathrm{O},-4.1686773767,3.4512054436,2.4656946789$ $\mathrm{H}, \mathrm{0},-4.3455727682,3.9928713105,0.9243239336$ $\mathrm{N}, 0,-3.4034485409,2.2681363628,1.0870479508$ C, $0,-2.1278279709,1.9170967198,0.7945825017$ $\mathrm{N}, 0,-1.8589048414,0.7258062268,0.3024412675$ $\mathrm{N}, 0,-1.1665936931,2.8096376435,1.071626826$ $\mathrm{H}, \mathrm{O},-1.4686150582,3.7453107268,1.2927917939$ $\mathrm{H}, \mathrm{O},-0.2589947978,2.6624291595,0.6530722467$ $\mathrm{H}, 0,-2.6966136859,0.1742441851,0.1642333569$ $\mathrm{H}, \mathrm{O},-4.1408027953,1.6055033121,0.8913690221$ $\mathrm{Cu}, 0,-0.264234789,-0.1438279639,-0.4042916335$ $0,0,1.0141337957,1.6826259171,-0.4729500649$ $\mathrm{H}, 0,1.8969199179,1.4087065388,-0.2003779137$ $\mathrm{H}, 0,1.1108088205,1.9326701736,-1.3989315799$ $0,0,-1.5937213791,-1.8980869796,-0.6836585697$ $\mathrm{H}, \mathrm{O},-1.0983306784,-2.612970554,-1.0982139193$ $\mathrm{H}, \mathrm{O},-1.8622647742,-2.2526751151,0.1713016771$



\section{$[23][\mathrm{Cu}(\mathrm{AGD}) 2]^{2+}$}

Charge $=2$ Multiplicity $=2$

$\mathrm{Cu}, 0,-0.0000268713,0.0000842146,-0.0005100389$

$\mathrm{N}, 0,1.344649206,-1.6195356725,0.0499819319$

$\mathrm{H}, \mathrm{O}, 1.2604995597,-2.0888218592,0.9491156118$

$\mathrm{H}, 0,1.1463401657,-2.2952379427,-0.681598117$

$\mathrm{N}, 0,1.6961249172,0.9607848346,0.0025800777$

$\mathrm{H}, \mathrm{0}, 1.8601391967,1.9577876515,0.0315173619$

$\mathrm{C}, 0,2.7782127354,0.2337375583,-0.0043550857$

$\mathrm{N}, 0,2.6384715628,-1.1198837807,-0.1184123841$

$\mathrm{H}, 0,3.3943095664,-1.7088324269,0.2047822336$

$\mathrm{N}, 0,4.0342369268,0.7050295353,0.0936139226$

$\mathrm{H}, 0,4.1743284529,1.6898234899,-0.0677697742$

$\mathrm{H}, \mathrm{O}, 4.8024133421,0.0978231879,-0.1474504332$

$\mathrm{N}, 0,-1.3446974939,1.6197149327,-0.0509177707$

$\mathrm{H}, \mathrm{O},-1.1462715461,2.295476894,0.6805747727$

$\mathrm{H}, 0,-1.2606808328,2.0889241544,-0.9501040325$

$\mathrm{N}, 0,-2.6384930944,1.1200981167,0.1177951117$

$\mathrm{C}, 0,-2.7782674791,-0.2335534989,0.0041361617$

$\mathrm{N}, 0,-1.6961857191,-0.9606090041,-0.0028482844$

$\mathrm{N}, 0,-4.0343181791,-0.7048598709,-0.0934045334$

$\mathrm{H}, \mathrm{O},-4.1743813477,-1.6896068182,0.0682919262$

$\mathrm{H}, 0,-4.8024274232,-0.097574085,0.1476748331$

$\mathrm{H}, \mathrm{O},-1.8602126916,-1.9576182284,-0.0314949707$

$\mathrm{H}, \mathrm{0},-3.3943948342,1.7089821495,-0.2053685202$

\section{$[24]\left[\mathrm{Cu}(\mathrm{AG})_{2}\right]^{2+}$ (mirror image)}

Charge $=2$ Multiplicity $=2$

$\mathrm{Cu}, 0,0.0232515895,-0.1534066206,-0.0253631415$ $\mathrm{N}, 0,-1.2646397875,-1.753649724,0.3771330313$

$\mathrm{H}, \mathrm{O},-1.3051714619,-2.3702299217,-0.4321235206$ $\mathrm{H}, \mathrm{O},-0.9455265299,-2.2991820052,1.1723073126$ $\mathrm{N}, 0,-1.7039054032,0.7796407023,-0.0335549664$ $\mathrm{H}, 0,-1.8989608487,1.7541886342,-0.2170805817$ $\mathrm{C}, 0,-2.7399732126,0.0682227754,0.3074431099$ $\mathrm{N}, 0,-2.5325678536,-1.2358631589,0.6568161039$ $\mathrm{H}, \mathrm{O},-3.3074753818,-1.8821233489,0.588756756$ $\mathrm{N}, 0,-4.0125509224,0.5065524827,0.3404348152$ $\mathrm{H}, 0,-4.1512458152,1.5047956375,0.3505210083$ $\mathrm{H}, \mathrm{O},-4.6960324408,-0.0397299361,0.8418760845$ $\mathrm{N}, 0,1.8290354104,-1.2101230176,-0.0812664774$ $\mathrm{H}, 0,1.9446304472,-1.813694698,0.7274102673$ $\mathrm{H}, 0,1.8405277401,-1.7950860153,-0.9145943395$ $\mathrm{N}, 0,2.8822710437,-0.2906526846,-0.1208587033$ C, $0,2.5375912562,1.004097709,-0.3918136495$ $\mathrm{N}, 0,1.2770827847,1.3246043849,-0.3307180504$ $\mathrm{N}, 0,3.5430485177,1.8437561897,-0.6992201036$ $\mathrm{H}, \mathrm{0}, 3.3518148496,2.8315235291,-0.641537546$ $\mathrm{H}, 0,4.4890201269,1.559952931,-0.4961830706$ $\mathrm{H}, \mathrm{O}, 1.0783784425,2.3015435201,-0.4965173032$ $\mathrm{H}, 0,3.7614054592,-0.635082365,-0.4838610153$
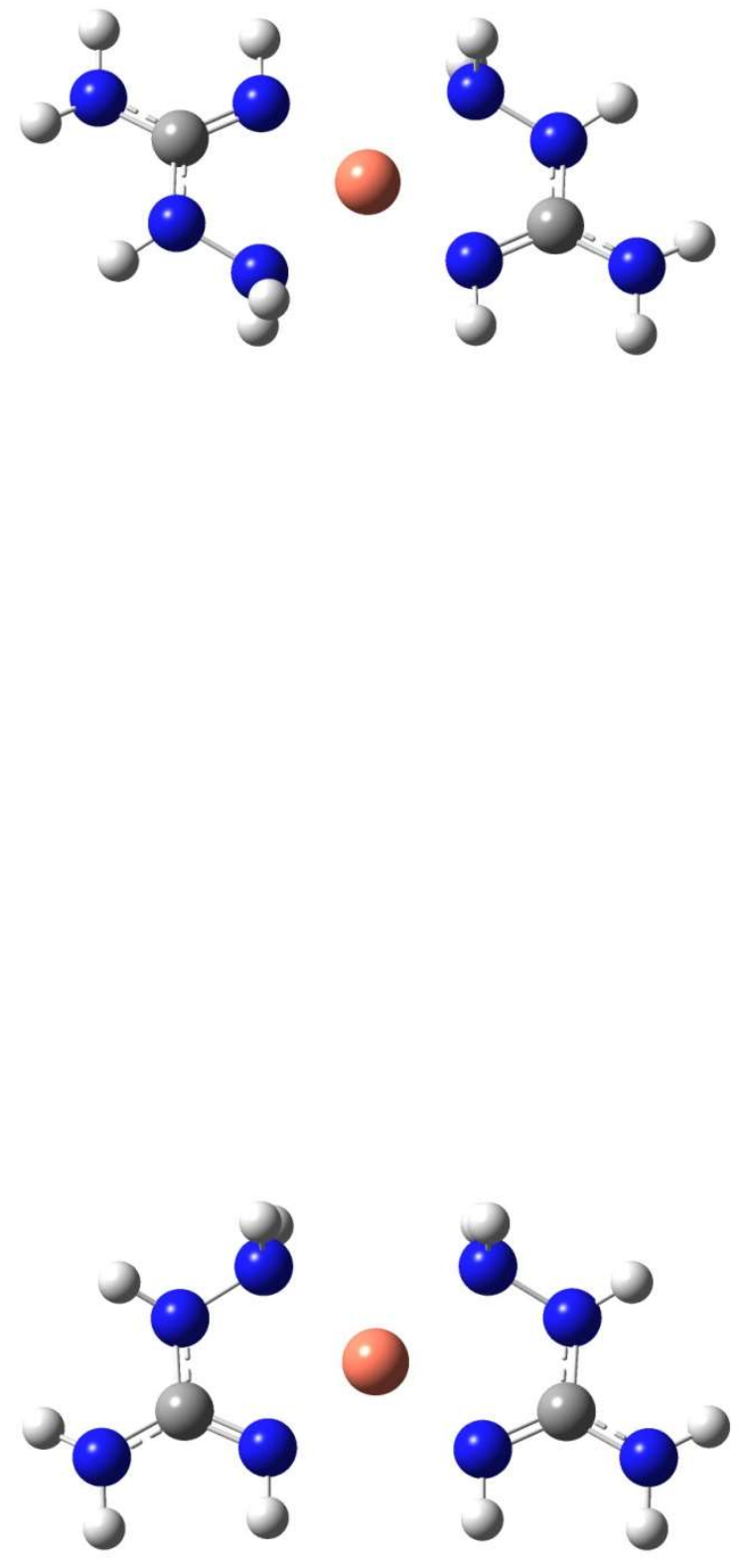


\section{$[25]\left[\mathrm{Cu}\left(\mathrm{AG}_{\mathrm{D}}\right)_{2}\left(\mathrm{H}_{2} \mathrm{O}\right)\right]^{2+}$}

Charge $=2$ Multiplicity $=2$

$\mathrm{Cu}, 0,0.3051133076,-0.0246268025,-0.1162470006$ $\mathrm{N}, 0,-1.0509058828,-1.6402093584,0.1743594162$ $\mathrm{H}, \mathrm{O},-0.8965249025,-2.3117883238,-0.5742050029$ $\mathrm{H}, \mathrm{O},-0.8980992198,-2.1082303017,1.0619363458$ $\mathrm{N}, 0,-1.4096196609,0.8711160014,-0.4055764198$ $\mathrm{H}, 0,-1.5720594106,1.8255676661,-0.6969993179$ $\mathrm{C}, 0,-2.4870975907,0.1439920457,-0.3073770025$ $\mathrm{N}, 0,-2.353115539,-1.1417004284,0.1266678468$ $\mathrm{H}, \mathrm{O},-3.0938141889,-1.8017850685,-0.0682840518$ $\mathrm{N}, 0,-3.7337083634,0.5568798283,-0.6018996595$ $\mathrm{H}, 0,-3.8862939338,1.5497055999,-0.6807296306$ $\mathrm{H}, \mathrm{O},-4.5146759946,0.0100152831,-0.2737888459$ $\mathrm{N}, 0,1.2806854229,-1.061443992,-2.227780873$ $\mathrm{H}, 0,0.637285833,-1.6306670413,-2.7653611195$ $\mathrm{H}, \mathrm{O}, 1.6790713247,-0.3583456483,-2.8420232477$ $\mathrm{N}, 0,2.2859755223,-1.8710012019,-1.7156789843$ $\mathrm{C}, 0,2.6280255765,-1.7512050812,-0.4076587136$ $\mathrm{N}, 0,1.9106674783,-1.0229376338,0.4014886526$ $\mathrm{N}, 0,3.7530635732,-2.4084251118,-0.0289157574$ $\mathrm{H}, 0,3.8546592371,-2.5855155075,0.9582687782$ $\mathrm{H}, \mathrm{O}, 4.0817170532,-3.1513292032,-0.6274183662$ $\mathrm{H}, 0,2.2392477712,-1.0545874473,1.3573211345$ $\mathrm{H}, 0,2.9673201702,-2.2615526762,-2.3522472584$ $0,0,1.4262891398,1.8255435344,-0.3212882942$ $\mathrm{H}, 0,2.0508425785,1.9231732011,0.4057678535$ $\mathrm{H}, 0,0.8583850087,2.6014039674,-0.2640195918$

\section{$[26]\left[\mathrm{Cu}(\mathrm{AGD})_{2}\left(\mathrm{H}_{2} \mathrm{O}\right)_{2}\right]^{2+}$}

Charge $=2$ Multiplicity $=2$

$\mathrm{Cu}, 0,0.0784152418,-0.1418004841,0.3901393241$ $\mathrm{N}, 0,-3.0222524382,-0.645759912,1.4939212135$ $\mathrm{H}, \mathrm{O},-3.1597083436,-1.4899164793,0.9442201951$ $\mathrm{H}, \mathrm{O},-3.5967063345,-0.7307455958,2.325511443$ $\mathrm{N}, 0,-1.3663102675,1.1439462651,-0.0098753489$ $\mathrm{H}, \mathrm{O},-0.9813389032,1.8932981134,-0.5738169334$ $\mathrm{C}, 0,-2.6663299367,1.2655372812,0.0598793467$ $\mathrm{N}, 0,-3.4782850153,0.4604076576,0.7859481733$ $\mathrm{H}, \mathrm{O},-4.465834482,0.5027164917,0.5751810927$ $\mathrm{N}, 0,-3.333074378,2.2257587621,-0.6420036608$ $\mathrm{H}, 0,-2.7718726713,2.9864837738,-0.991271968$ $\mathrm{H}, \mathrm{O},-4.2404135435,2.5044803545,-0.2994128826$ $\mathrm{N}, 0,2.26896953,1.1433375253,0.9818888586$ $\mathrm{H}, 0,2.52610447,1.9068831242,0.3678164288$ $\mathrm{H}, \mathrm{O}, 2.2588706648,1.4913189778,1.9358464132$ $\mathrm{N}, 0,3.2067433527,0.1266303996,0.85334041$ $\mathrm{C}, 0,2.7809342101,-1.1594662278,0.7828443527$ $\mathrm{N}, 0,1.5155792462,-1.4391835821,0.6339694649$ $\mathrm{N}, 0,3.7417214916,-2.1100411866,0.9080415177$ $\mathrm{H}, \mathrm{O}, 3.5088304696,-3.0232948366,0.550236654$ $\mathrm{H}, 0,4.6976485185,-1.8276415024,0.7518196359$ $\mathrm{H}, 0,1.3397287705,-2.4319579775,0.5523967942$ $\mathrm{H}, \mathrm{O}, 4.1498597432,0.2951731945,1.1773525687$ $0,0,-0.4448497786,-0.2572740015,2.4926448434$ $\mathrm{H}, \mathrm{O},-0.0619822103,-1.064779605,2.8498914688$ $\mathrm{H}, \mathrm{O},-1.3903014371,-0.4648818628,2.3164112039$ $0,0,0.5609198653,-0.1455750907,-1.7866876181$ $\mathrm{H}, 0,1.5108687795,-0.2781239267,-1.8760461065$ $\mathrm{H}, 0,0.387541526,0.7012531697,-2.2115443448$
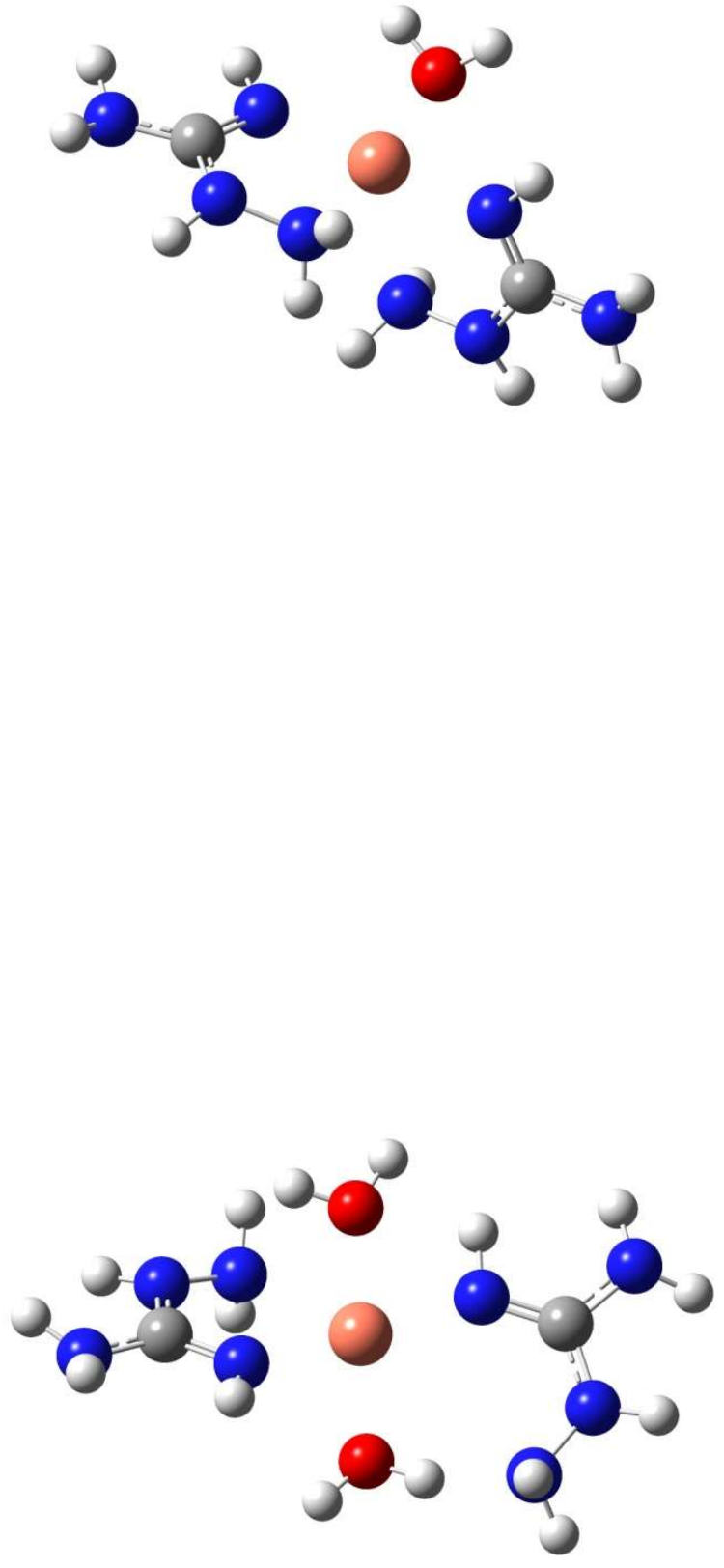


\section{$[27]\left[\mathrm{Cu}\left(\mathrm{AG}_{\mathrm{A}}\right)(\mathrm{AG})\right]^{2+}$}

Charge $=2$ Multiplicity $=2$

$\mathrm{Cu}, 0,0.0292978958,-0.0094812973,0.0298131362$

$\mathrm{N}, 0,-1.2847437179,-1.6148302122,-0.2045263373$

$\mathrm{H}, 0,-1.205629766,-1.9704380615,-1.1549758261$

$\mathrm{H}, \mathrm{0},-1.0623013837,-2.3695090677,0.4380026344$

$\mathrm{N}, 0,-1.667211751,0.9284219015,0.1800974752$

$\mathrm{H}, \mathrm{0},-1.8407105975,1.9181533805,0.2872630362$ C, $0,-2.741169479,0.1917880179,0.1081564459$ $\mathrm{N}, 0,-2.5832859916,-1.1623177146,0.041186369$ $\mathrm{H}, \mathrm{O},-3.3352104983,-1.7168720705,-0.3459105312$ $\mathrm{N}, 0,-4.0017350829,0.6559424403,0.0935455491$ $\mathrm{H}, 0,-4.1508343165,1.6118801554,0.3750209884$ $\mathrm{H}, \mathrm{O},-4.7611652662,0.0139549584,0.2602576886$ $\mathrm{N}, 0,4.6567407972,-0.7829718361,-0.1326347734$ $\mathrm{H}, 0,5.255108849,-0.9053078397,0.677331941$ $\mathrm{H}, 0,5.2515022671,-0.6188663755,-0.9378094365$ $\mathrm{N}, 0,3.8614104544,0.337355159,0.0670832678$ $\mathrm{C}, 0,2.5362697076,0.2180635498,0.0584180738$ $\mathrm{N}, 0,1.8179208594,-0.8396981311,-0.1136905518$ $\mathrm{N}, 0,1.7255275537,1.3727206418,0.2600418144$ $\mathrm{H}, 0,1.8975685107,1.7907245608,1.1708345559$ $\mathrm{H}, 0,1.9032035122,2.0787220518,-0.449733094$ $\mathrm{H}, \mathrm{O}, 2.3134631897,-1.7105239306,-0.2641704448$ $\mathrm{H}, \mathrm{0}, 4.2752202738,1.2491106595,0.2184901393$
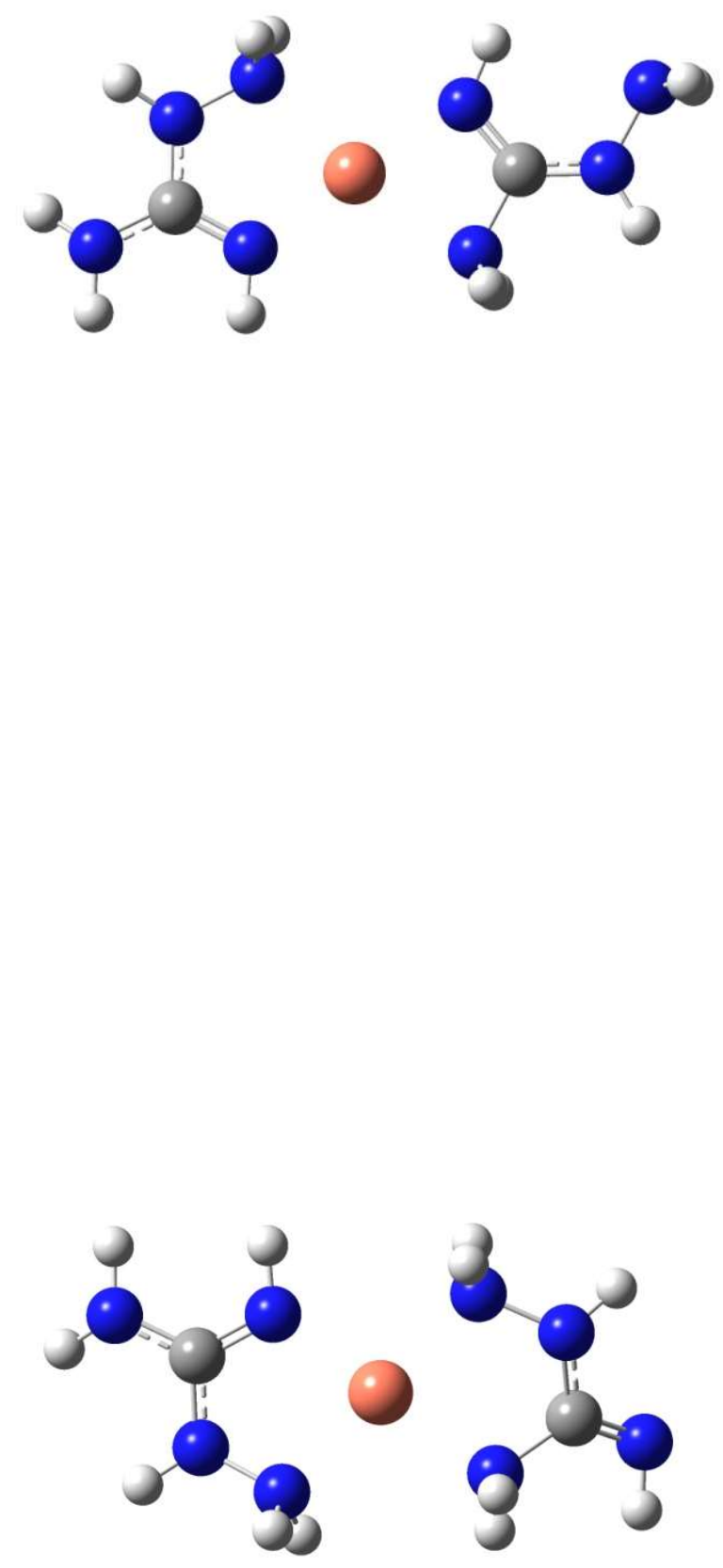

Charge $=2$ Multiplicity $=2$

$\mathrm{Cu}, 0,-0.0263315854,0.0090218947,0.2064678161$

$\mathrm{N}, 0,-1.3525287272,-1.578765928,0.2470436672$

$\mathrm{H}, \mathrm{O},-1.153185015,-2.1760660101,-0.5526709132$ $\mathrm{H}, \mathrm{O},-1.231779165,-2.1322470667,1.0899969245$

$\mathrm{N}, 0,-3.8667565216,0.5800670058,-0.8630918662$

$\mathrm{H}, 0,-3.8944128735,1.5946325053,-0.9162786315$

C, $0,-2.8539664899,0.2047453956,-0.1872129612$

$\mathrm{N}, 0,-2.6758495871,-1.1082803516,0.1817144418$

$\mathrm{H}, 0,-3.3323414354,-1.7543831572,-0.2367017771$

$\mathrm{N}, 0,-1.8015667173,1.0341253042,0.3168765966$

$\mathrm{H}, \mathrm{0},-1.931241985,1.1720533895,1.3190206426$

$\mathrm{H}, 0,-1.8237185638,1.9470545148,-0.126473991$

$\mathrm{N}, 0,1.3078445282,1.6013911843,0.2026543111$

$\mathrm{H}, 0,1.0267592229,2.3184831659,-0.4601195393$

$\mathrm{H}, \mathrm{O}, 1.3363221139,2.0205024903,1.1301730567$

$\mathrm{N}, 0,2.5675413764,1.1116549813,-0.1508014929$

$\mathrm{C}, 0,2.7192849745,-0.2441972213,-0.1133314565$

$\mathrm{N}, 0,1.6460900567,-0.9744393475,0.0149241864$

$\mathrm{N}, 0,3.9746757054,-0.7154959542,-0.2100680557$

$\mathrm{H}, \mathrm{O}, 4.0843053082,-1.6939145447,-0.4254292111$

$\mathrm{H}, \mathrm{O}, 4.6987722126,-0.1006589673,-0.5489857425$

$\mathrm{H}, 0,1.8015137263,-1.9723561577,-0.0285894939$

$\mathrm{H}, \mathrm{O}, 3.3598157709,1.6950295947,0.0830240491$ 


\section{[29] $[\mathrm{Cu}(\mathrm{AGc})(\mathrm{AGD})]^{2+}$}

Charge $=2$ Multiplicity $=2$

$\mathrm{Cu}, 0,0.0424816967,-0.0211842874,0.1407783039$

$\mathrm{N}, 0,-1.3052804141,-1.5954851195,0.1985037187$

$\mathrm{H}, \mathrm{O},-1.1362683479,-2.1843551367,-0.6140666401$

$\mathrm{H}, 0,-1.1706083913,-2.1623265725,1.0307924876$

$\mathrm{N}, 0,-3.8074333685,0.7805903158,-0.6589463308$

$\mathrm{H}, \mathrm{O},-4.4516042363,0.0696481,-1.0005773565$

$\mathrm{C}, 0,-2.8042832608,0.2291551808,-0.0983325782$

$\mathrm{N}, 0,-2.622791936,-1.106634082,0.1701764469$

$\mathrm{H}, \mathrm{O},-3.2989899938,-1.7353954879,-0.2435334$

$\mathrm{N}, 0,-1.7168522029,1.0173703694,0.3856265677$

$\mathrm{H}, 0,-1.7904654774,1.1098154868,1.3983653308$

$\mathrm{H}, \mathrm{O},-1.7714199839,1.9491466204,-0.0120436709$

$\mathrm{N}, 0,1.362809616,1.5741567694,0.0460268841$

$\mathrm{H}, 0,1.0869694204,2.237284414,-0.672888647$

$\mathrm{H}, 0,1.369450991,2.0610760098,0.9402608745$

$\mathrm{N}, 0,2.6362460367,1.0759249865,-0.2456260214$

C, $0,2.793294816,-0.2747427329,-0.114460057$

$\mathrm{N}, 0,1.7163220161,-1.0049260594,-0.0189331619$

$\mathrm{N}, 0,4.0526089525,-0.7404663988,-0.0991670939$

$\mathrm{H}, 0,4.1882679789,-1.7280864917,-0.2461027499$

$\mathrm{H}, 0,4.8068284795,-0.1338192391,-0.3815923337$

$\mathrm{H}, \mathrm{O}, 1.8797592644,-2.0011758215,0.0314551165$

$\mathrm{H}, 0,3.4149668246,1.6746470368,-0.0045099995$

\section{$[30][\mathrm{Cu}(\mathrm{AGc})(\mathrm{AG})]^{2+}$ (mirror image)}

Charge $=2$ Multiplicity $=2$

$\mathrm{Cu}, 0,0.0325704636,-0.0834108157,-0.0704998908$

$\mathrm{N}, 0,1.79089399,-1.1569453247,0.0890267736$

$\mathrm{H}, \mathrm{O}, 1.8077600673,-1.5820058972,1.0145282864$

$\mathrm{H}, 0,1.8395607613,-1.9052827492,-0.5963692269$

$\mathrm{N}, 0,3.4770211619,1.9613281821,0.3552298713$

$\mathrm{H}, 0,4.3070606769,1.5415982465,0.769897966$

$\mathrm{C}, 0,2.6764852263,1.0498417206,-0.0360662453$

$\mathrm{N}, 0,2.901438776,-0.3073559134,-0.0641761968$

$\mathrm{H}, 0,3.7375948625,-0.6202688885,0.4128566617$

$\mathrm{N}, 0,1.3982139052,1.3840668387,-0.5693060745$

$\mathrm{H}, 0,1.4182310449,1.295107201,-1.5848096032$

$\mathrm{H}, 0,1.17407628,2.3476523563,-0.345812648$

$\mathrm{N}, 0,-1.3249238666,-1.651670364,0.1768726475$

$\mathrm{H}, \mathrm{O},-1.1436791967,-2.4076091415,-0.4769379494$

$\mathrm{H}, \mathrm{O},-1.2431891823,-2.0228074613,1.1216808245$

$\mathrm{N}, 0,-2.611593604,-1.1494361922,-0.0403758589$

$\mathrm{C}, 0,-2.7294767512,0.2112234327,-0.0623593856$

$\mathrm{N}, 0,-1.6353427764,0.9186199393,-0.1155929248$

$\mathrm{N}, 0,-3.9756134516,0.7113297718,-0.0272678249$

$\mathrm{H}, \mathrm{O},-4.0974037697,1.675483284,-0.2939301829$

$\mathrm{H}, \mathrm{O},-4.7537009085,0.0944220646,-0.2016914897$

$\mathrm{H}, \mathrm{O},-1.7736975355,1.9159096088,-0.2028389214$

$\mathrm{H}, \mathrm{0},-3.3742671635,-1.6892208887,0.3478274021$
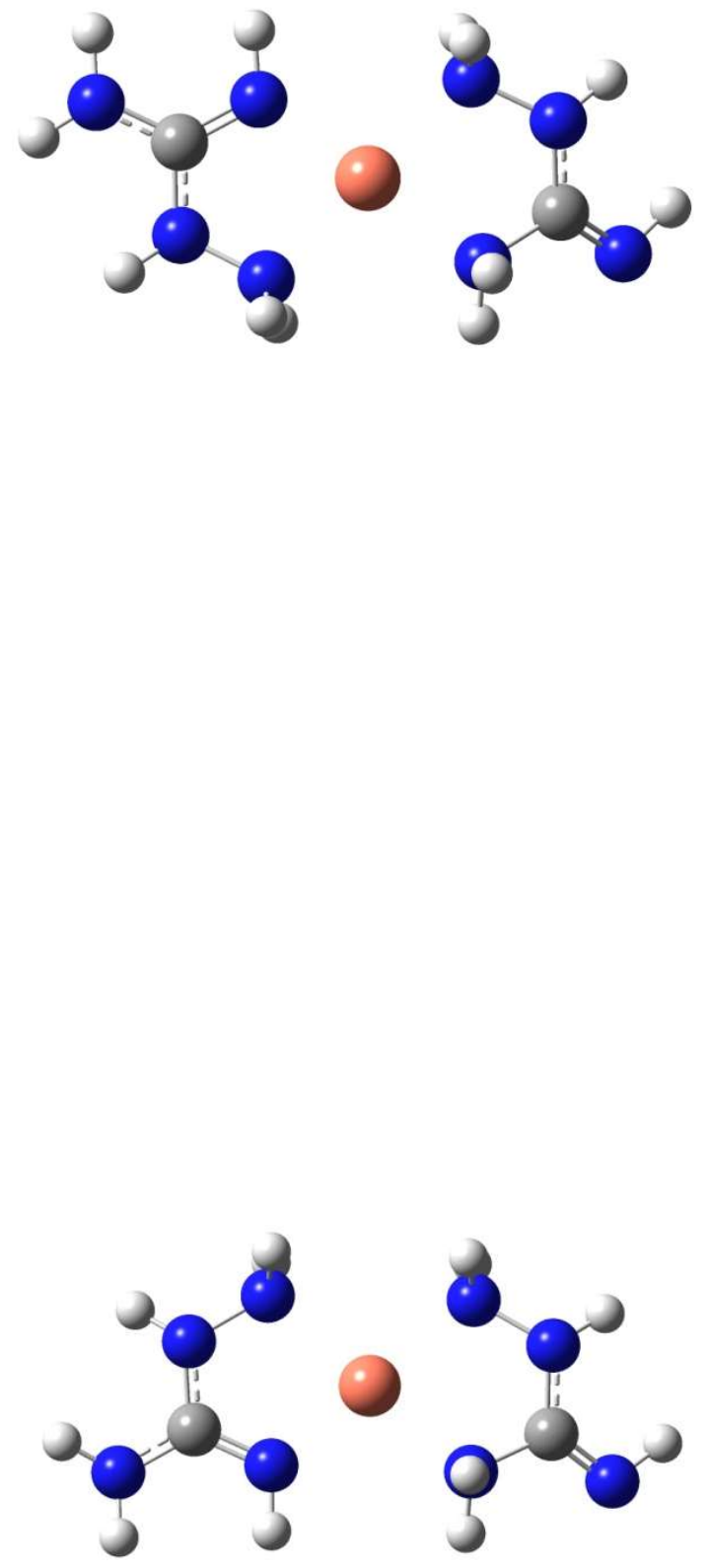


\section{$[31]\left[\mathrm{Cu}(\mathrm{AGH})\left(\mathrm{H}_{2} \mathrm{O}\right)\right]^{2+} \cdot 2 \mathrm{H}_{2} \mathrm{O}$}

\begin{abstract}
Charge $=2$ Multiplicity $=1$ $\mathrm{Cu}, 0,-1.598875885,0.5617071647,-0.1398184512$ $\mathrm{N}, 0,-0.1976060621,-0.5452481223,-1.0654325908$ $\mathrm{H}, \mathrm{O},-0.2967892137,-1.5605695619,-0.9333756499$ $\mathrm{H}, \mathrm{O},-0.4173107747,-0.356142512,-2.0404391539$ $\mathrm{N}, 0,1.1603949857,-0.2138399607,-0.9233711722$ C, $0,1.7766994219,-0.1779250968,0.263834134$ $\mathrm{N}, 0,1.1206021737,-0.3687490538,1.3988279371$ $\mathrm{N}, 0,3.0854115903,0.0644128383,0.2807240601$ $\mathrm{H}, 0,3.5970165336,-0.0226643784,1.1441063482$ $\mathrm{H}, 0,3.606032155,0.1029448057,-0.581016429$ $\mathrm{H}, 0,1.5925200575,-0.1995372305,2.2734756195$ $\mathrm{H}, 0,1.7380311162,-0.3289041962,-1.7456619139$ $0,0,-3.0199200155,1.7158643975,0.6758593929$ $\mathrm{H}, \mathrm{O},-3.4650618259,1.2700929756,1.4044880801$ $\mathrm{H}, \mathrm{O},-2.6488388313,2.5159092447,1.0632998727$ $\mathrm{H}, 0,0.116732374,-0.4553832901,1.4116918061$ $0,0,-0.0116643273,-3.4354331332,-0.6302591601$ $\mathrm{H}, \mathrm{O},-0.7600513185,-3.9897214836,-0.869201194$ $0,0,0.6493430362,3.1265087027,0.6330263289$ $\mathrm{H}, 0,0.0659330597,2.3845895641,0.4423121417$ $\mathrm{H}, 0,0.1890705662,-3.6867861933,0.2759216934$ $\mathrm{H}, 0,1.5190921841,2.8051725195,0.3789333005$
\end{abstract}

\section{$[32]\left[\mathrm{Cu}(\mathrm{AGc})\left(\mathrm{H}_{2} \mathrm{O}\right)\right]^{+} \cdot \mathrm{H}_{2} \mathrm{O}$}
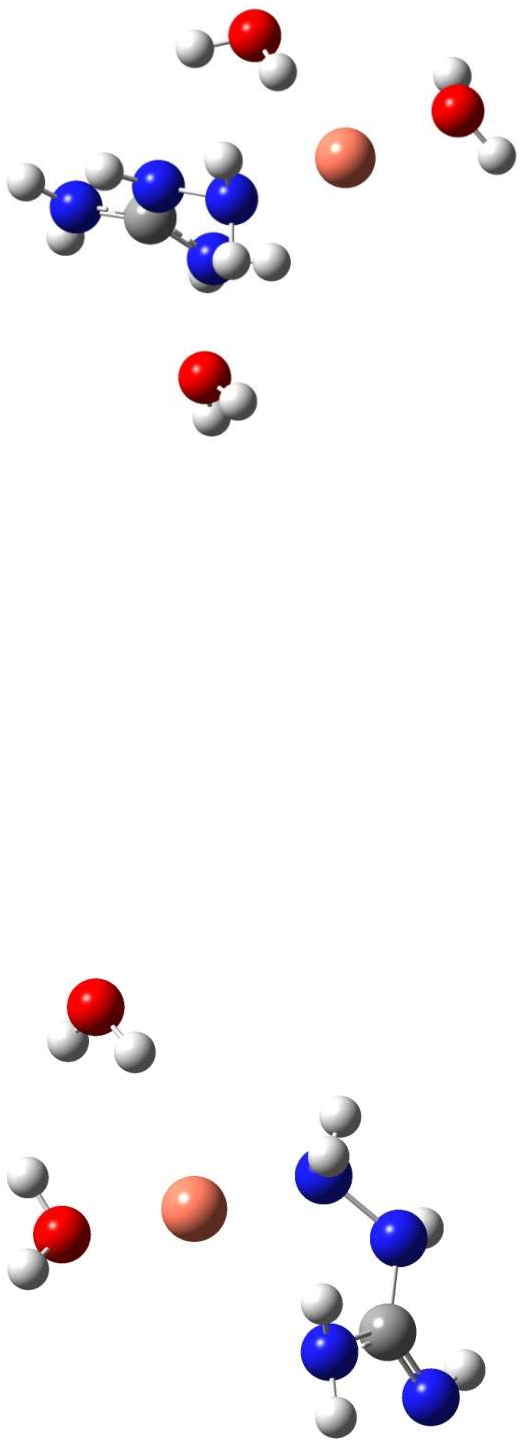


\section{$[33]\left[\mathrm{Cu}(\mathrm{AGc})\left(\mathrm{H}_{2} \mathrm{O}\right)\right]^{+} \cdot 2 \mathrm{H}_{2} \mathrm{O}$}

Charge $=1$ Multiplicity $=1$ $\mathrm{N}, 0,4.500749164,0.5430972108,-0.0459485371$ $\mathrm{H}, 0,4.9787226886,0.4558117571,-0.9368723756$ $\mathrm{H}, 0,5.175035445,0.3774044249,0.6910094824$ $\mathrm{N}, 0,3.4894486029,-0.4071417244,0.0487295045$ $\mathrm{C}, 0,2.1797056046,-0.0327038291,-0.0630374194$ $\mathrm{N}, 0,1.2244387228,-0.9278937686,-0.1386498704$ $\mathrm{N}, 0,1.9239132645,1.2851991589,-0.0817381239$ $\mathrm{H}, 0,0.9769816408,1.6138000038,0.0640827167$ $\mathrm{H}, \mathrm{O}, 2.6964096329,1.9072960554,0.0932424315$ $\mathrm{H}, 0,1.5993011369,-1.8683679871,-0.1247462455$ $\mathrm{H}, 0,3.7014724185,-1.3634264386,-0.2024265447$ $\mathrm{Cu}, 0,-0.6594591931,-0.6471701273,-0.1884472298$ $0,0,-3.9496644316,-2.6896462216,0.2074130877$ $\mathrm{H}, \mathrm{O},-4.805345778,-2.7523453443,-0.2275871703$ $\mathrm{H}, \mathrm{O},-4.1465967257,-2.8108681904,1.1409933519$ $0,0,-0.7692640603,2.6170645054,0.3303756969$ $\mathrm{H}, \mathrm{O},-0.9319350071,3.3210048059,-0.3043412692$ $\mathrm{H}, \mathrm{O},-0.8168442717,3.0568641512,1.1841190308$ $0,0,-2.6159323986,-0.3420940903,-0.2831727083$ $\mathrm{H}, 0,-2.8951544406,0.3079100959,0.3689074657$ $\mathrm{H}, 0,-3.1251990148,-1.1562224478,-0.0816662736$
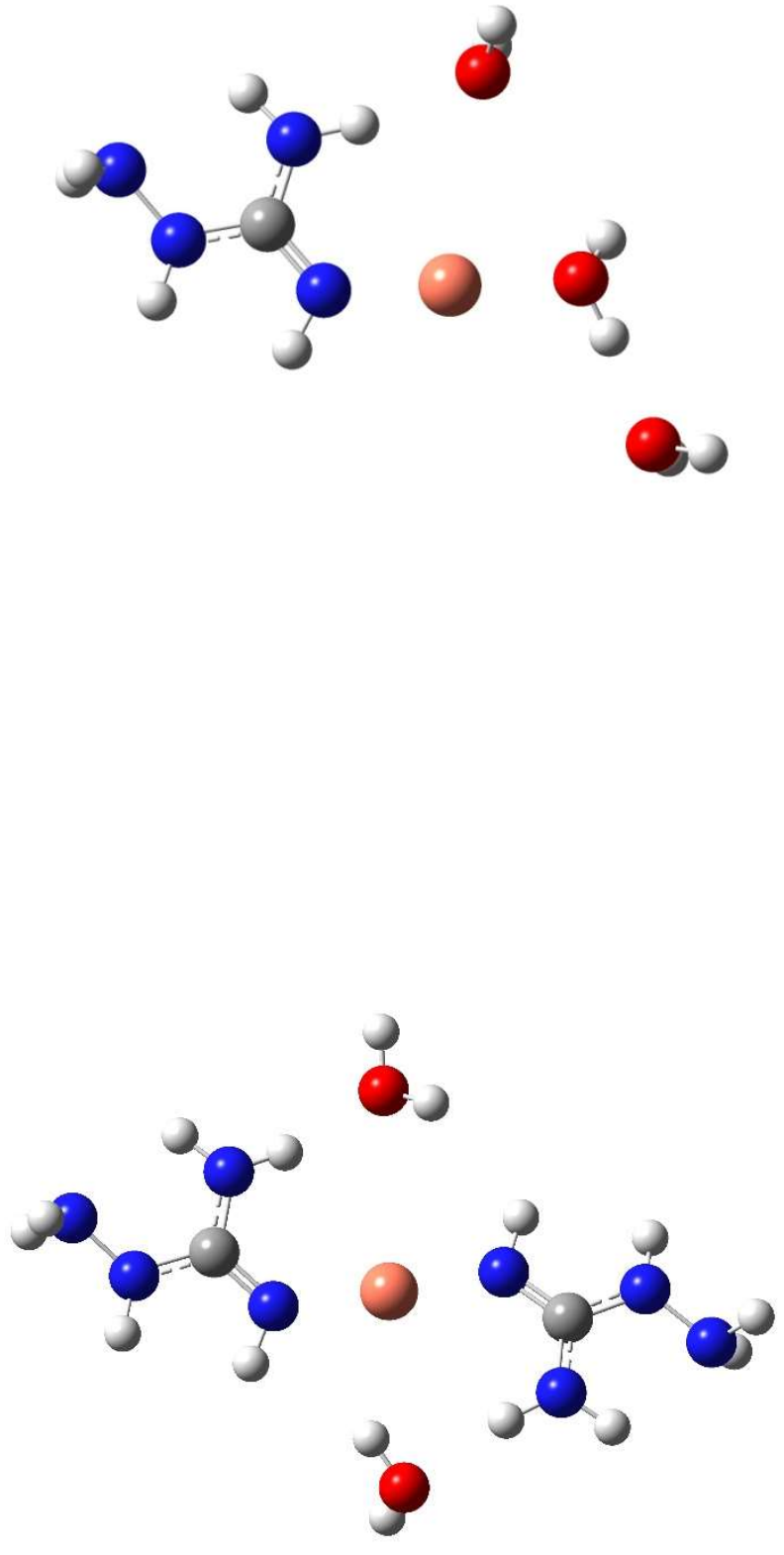

\section{$[34]\left[\mathrm{Cu}(\mathrm{AGc})_{2}\right]^{+} \cdot 2 \mathrm{H}_{2} \mathrm{O}$}

Charge $=1$ Multiplicity $=1$

$\mathrm{N}, 0,-5.367247043,-0.0235969127,0.1968677265$ $\mathrm{H}, 0,-5.7950434957,-0.4136617715,1.030054065$ $\mathrm{H}, \mathrm{O},-6.0130240421,-0.141014857,-0.5745955723$ $\mathrm{N}, 0,-4.1876485561,-0.6986529119,-0.0922258353$ $\mathrm{C}, 0,-2.9784262944,-0.091680277,0.0860758018$ $\mathrm{N}, 0,-1.857635177,-0.7553538841,-0.06829356$ $\mathrm{N}, 0,-2.9985827961,1.2093800041,0.4235573145$ $\mathrm{H}, \mathrm{0},-3.893935772,1.669743442,0.3761862546$ $\mathrm{H}, 0,-2.1602914175,1.7647249982,0.299587942$ $\mathrm{H}, \mathrm{O},-2.0375437986,-1.7236816674,-0.3063627762$ $\mathrm{H}, 0,-4.1943915193,-1.709142338,-0.1125519464$ $\mathrm{N}, 0,5.2921988156,0.0508965943,0.0797300582$ $\mathrm{H}, 0,5.9228818993,0.3837947223,0.7987108183$ $\mathrm{H}, \mathrm{O}, 5.6913550374,0.2841578216,-0.8234345522$ $\mathrm{N}, 0,4.0604760267,0.6795605275,0.2309005089$ $\mathrm{C}, 0,2.9055108602,-0.0508522402,0.187157263$ $\mathrm{N}, 0,1.7364577606,0.5369332361,0.1029551768$ $\mathrm{N}, 0,3.0329388957,-1.3828046745,0.3021494144$ $\mathrm{H}, 0,3.9606054682,-1.7608950035,0.1935023465$ $\mathrm{H}, 0,2.2343752173,-1.9778690332,0.1189176751$ $\mathrm{H}, \mathrm{0}, 1.8400238956,1.5432102812,0.0411614901$ $\mathrm{H}, 0,3.9822224265,1.6537813508,-0.0268236093$ $\mathrm{Cu}, 0,-0.0458818024,-0.1517565802,0.0418612843$ $0,0,0.8083688958,-3.4008373649,-0.0503308664$ $\mathrm{H}, 0,0.1256722363,-2.7502321313,0.1439248794$ $\mathrm{H}, 0,0.6404859858,-3.6483820286,-0.9640481141$ $0,0,-0.7613597183,3.2047728684,0.124927082$ $\mathrm{H}, \mathrm{O},-0.9882567238,4.089389404,0.4257069456$ $\mathrm{H}, \mathrm{O}, 0.0332327354,2.9847734254,0.6194357853$ 


\section{$[35]\left[\mathrm{Cu}\left(\mathrm{AG}_{\mathrm{A}}\right)_{2}\right]^{+} \cdot 2 \mathrm{H}_{2} \mathrm{O}$}

Charge $=1$ Multiplicity $=1$

$\mathrm{N}, 0,4.4258644997,-1.3339751372,0.0258994417$ $\mathrm{H}, 0,5.0356807386,-1.6277794685,0.7790457408$ $\mathrm{H}, \mathrm{O}, 4.8870791537,-1.5383003954,-0.854258066$ $\mathrm{N}, 0,4.1955580249,0.0313376126,0.1351733059$ $\mathrm{C}, 0,2.9148222976,0.4982999504,0.0297953899$ $\mathrm{N}, 0,1.8804946079,-0.2947268841,0.0384445797$ $\mathrm{N}, 0,2.7964898788,1.8427870988,-0.0926298704$ $\mathrm{H}, 0,3.5921593443,2.4021787994,0.1725865906$ $\mathrm{H}, \mathrm{O}, 1.8936671679,2.2508557641,0.1249581156$ $\mathrm{H}, 0,2.2107281188,-1.2541580763,0.0816773435$ $\mathrm{H}, 0,4.9441091685,0.6659465198,-0.1085600506$ $\mathrm{N}, 0,-4.4438724186,1.3118790865,-0.1109990007$ $\mathrm{H}, 0,-5.0677898191,1.6305400532,0.6200147184$ $\mathrm{H}, \mathrm{O},-4.8943780484,1.4741334856,-1.0055085832$ $\mathrm{N}, 0,-4.2024817184,-0.0459712444,0.0594190702$ $\mathrm{C}, 0,-2.9166457418,-0.5040629861,-0.0363447808$ $\mathrm{N}, 0,-1.8911607617,0.2998874075,-0.0648974567$ $\mathrm{N}, 0,-2.7851749502,-1.8498741367,-0.1097540714$ $\mathrm{H}, 0,-3.5704649802,-2.4102222012,0.1824168924$ $\mathrm{H}, \mathrm{O},-1.8716621021,-2.2428433345,0.0903577321$ $\mathrm{H}, \mathrm{O},-2.2324276185,1.2562485354,-0.0607441822$ $\mathrm{H}, \mathrm{O},-4.9423304913,-0.6945386493,-0.1744184734$ $\mathrm{Cu}, 0,-0.0042525911,0.0082947141,-0.0216774486$ $0,0,-0.1914601982,-3.3288295853,0.3787374987$ $\mathrm{H}, \mathrm{O},-0.1984177518,-4.1907037136,-0.0474909557$ $\mathrm{H}, 0,0.4207429647,-2.8101870074,-0.1515288706$ $0,0,0.2622596577,3.3639381507,0.5545158373$ $\mathrm{H}, 0,-0.337730065,2.6158166654,0.6327176285$ $\mathrm{H}, \mathrm{O},-0.0725993665,3.8533579766,-0.2022490747$

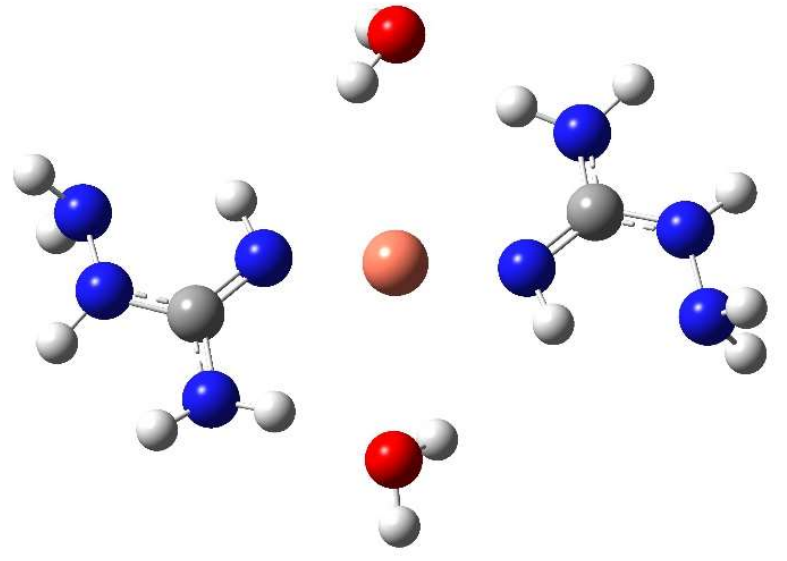

\section{[36] [Cu(AGc) 2$]^{+}$(mirror image)}

Charge $=1$ Multiplicity $=1$

$\mathrm{N}, 0,2.0303177062,-1.3938897348,-0.0241004008$ $\mathrm{H}, \mathrm{O}, 2.2942225102,-2.067822218,0.6905669772$ $\mathrm{H}, \mathrm{O}, 2.3744269971,-1.812464267,-0.8855448897$ $\mathrm{N}, 0,2.8479560774,-0.2434016165,0.2404158362$ C, $0,2.5319090109,0.961464391,-0.3811862256$ $\mathrm{N}, 0,2.7719492576,2.1144603095,0.1440901162$ $\mathrm{N}, 0,1.9809914244,0.8525862174,-1.6274573952$ $\mathrm{H}, \mathrm{O}, 2.2189831263,0.0436650189,-2.1802212974$ $\mathrm{H}, \mathrm{O}, 1.9807706944,1.7154190404,-2.1483956387$ $\mathrm{H}, 0,3.0717055689,1.9993634525,1.1083130411$ $\mathrm{H}, 0,2.9536967616,-0.1290583589,1.2394284218$ $\mathrm{N}, 0,-1.9668406947,-1.336961871,-0.0151176746$ $\mathrm{H}, \mathrm{O},-2.1382619339,-1.7954712331,-0.9067107811$ $\mathrm{H}, 0,-2.2863728709,-1.9912371436,0.6936695738$ $\mathrm{N}, 0,-2.8134484122,-0.199559008,0.0254785766$ C, $0,-2.347806496,1.0500677269,0.3993425119$ $\mathrm{N}, 0,-2.8696783579,2.1549168045,-0.015686168$ $\mathrm{N}, 0,-1.3078365435,1.0499088688,1.2881215579$ $\mathrm{H}, \mathrm{O},-1.2583371519,0.2790770268,1.936924692$ $\mathrm{H}, \mathrm{O},-1.1362407717,1.949442421,1.7092476961$ $\mathrm{H}, \mathrm{O},-3.539117945,1.9616319976,-0.7555810924$ $\mathrm{H}, \mathrm{O},-3.4743289308,-0.177945768,-0.7375803923$ $\mathrm{Cu}, 0,0.0408531332,-1.2126225764,-0.0074570751$

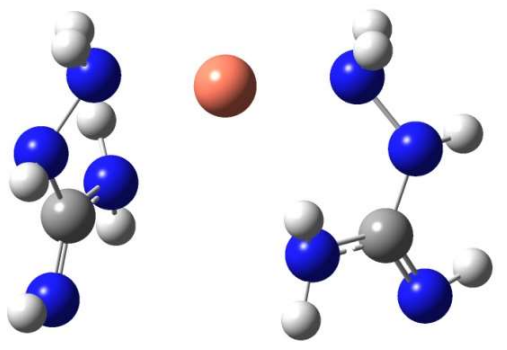




\section{$[37]\left[\mathrm{Cu}(\mathrm{AGc})_{2}\right]^{+}$}

Charge $=1$ Multiplicity $=1$

$\mathrm{N}, 0,1.9734540589,-0.2654999361,-1.5067770461$ $\mathrm{H}, 0,2.1773079724,-1.0146457327,-2.1638783095$ $\mathrm{H}, 0,2.4591746686,0.5374086285,-1.9002680375$ $\mathrm{N}, 0,2.6695525217,-0.6337790358,-0.305681618$ $\mathrm{C}, 0,2.3501329077,0.0250686894,0.8792203841$ $\mathrm{N}, 0,2.4076695073,-0.5329751932,2.0399252447$ $\mathrm{N}, 0,2.0022497241,1.3402732151,0.7428853128$ $\mathrm{H}, 0,2.3987733458,1.8541126712,-0.0289088942$ $\mathrm{H}, 0,1.9998529891,1.8510478299,1.6119574961$ $\mathrm{H}, 0,2.5684788036,-1.5311671156,1.9371021611$ $\mathrm{H}, 0,2.6281978075,-1.6375217841,-0.1912428639$ $\mathrm{N}, 0,-1.9738462299,0.2660640197,-1.5069917814$ $\mathrm{H}, 0,-2.1787925626,1.0147524268,-2.1642669966$ $\mathrm{H}, \mathrm{O},-2.4591231811,-0.537399741,-1.8998967752$ $\mathrm{N}, 0,-2.6697116841,0.6342298685,-0.3056265883$ $\mathrm{C}, 0,-2.3499938024,-0.0252905536,0.8788859766$ $\mathrm{N}, 0,-2.4051461378,0.5326692224,2.0397246806$ $\mathrm{N}, 0,-2.004496566,-1.3410284854,0.741891757$ $\mathrm{H}, 0,-2.4030522849,-1.8543710389,-0.0291826507$ $\mathrm{H}, \mathrm{O},-2.0002891663,-1.8518077374,1.6109373129$ $\mathrm{H}, 0,-2.5642568558,1.5311692429,1.9372778299$ $\mathrm{H}, 0,-2.6270719443,1.637871794,-0.190734271$ $\mathrm{Cu}, 0,-0.0001508916,0.0004297453,-1.4385793235$

\section{[38] $\left[\mathrm{Cu}(\mathrm{AGD})\left(\mathrm{H}_{2} \mathrm{O}\right)\right]^{+} \cdot \mathrm{H}_{2} \mathrm{O}$}

\footnotetext{
Charge $=1$ Multiplicity $=1$

$\mathrm{Cu}, 0,-0.5060083029,0.141794149,-0.2549729286$ $\mathrm{N}, 0,1.0837394292,-2.0818367737,0.0486721968$ $\mathrm{H}, \mathrm{O}, 0.9767521127,-2.6710975212,0.8653639159$ $\mathrm{H}, 0,1.0690945674,-2.6770720894,-0.7731379692$ $\mathrm{N}, 0,2.3022029791,-1.4228553558,0.1301613368$ $\mathrm{C}, 0,2.3941958502,-0.0925077985,-0.1393067496$ $\mathrm{N}, 0,1.3430271257,0.6562926891,-0.311106872$ $\mathrm{N}, 0,3.6724345404,0.3775328422,-0.2397008127$ $\mathrm{H}, 0,3.7809715809,1.3718263905,-0.1132844784$ $\mathrm{H}, 0,4.3962323656,-0.1699317156,0.2017280815$ $\mathrm{H}, \mathrm{0}, 1.6118669067,1.6198554246,-0.4618927517$ $\mathrm{H}, \mathrm{O}, 3.1490668376,-1.9694904728,0.0561834391$ $0,0,-2.4678383796,-0.2142950491,-0.2227721825$ $\mathrm{H}, \mathrm{O},-2.9814325938,0.5895756996,0.0056300498$ $0,0,-3.8414385902,2.0968258884,0.3292285504$ $\mathrm{H}, \mathrm{O},-4.7765262837,2.0413924644,0.1110913605$ $\mathrm{H}, 0,-2.6974126381,-0.8634756664,0.4488882787$ $\mathrm{H}, \mathrm{0},-3.8274975072,2.3363738944,1.2606685352$
}
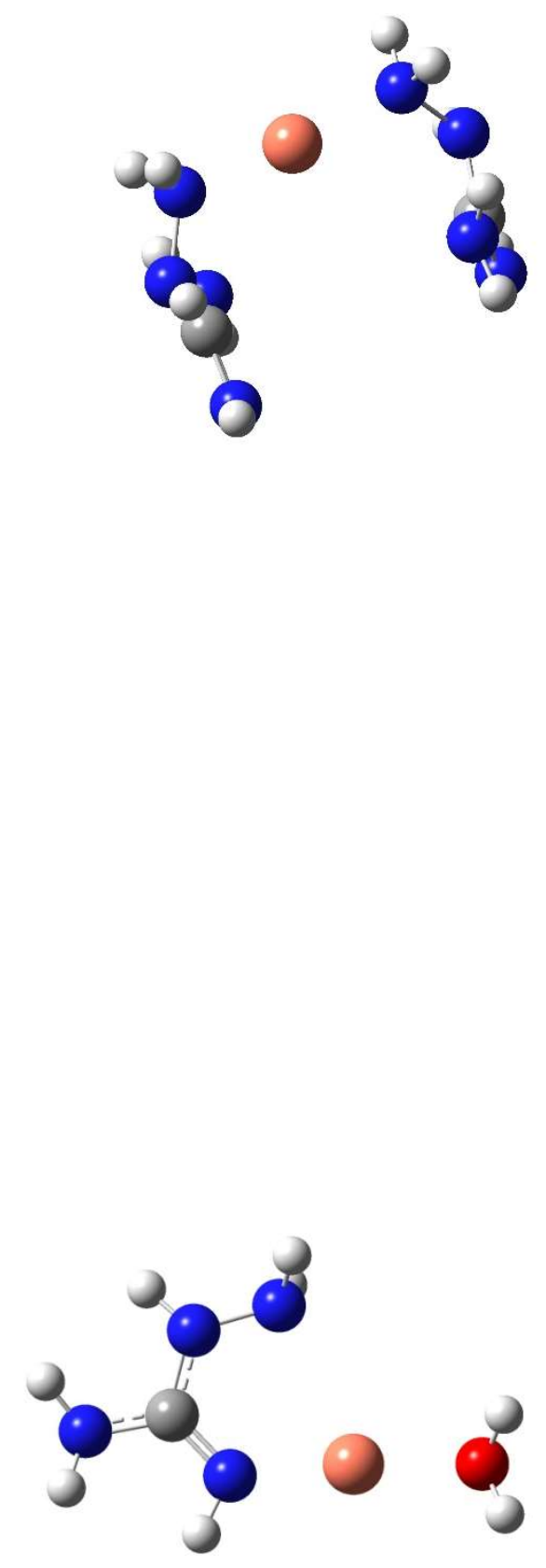


\section{[39] $[\mathrm{Cu}(\mathrm{AGc})(\mathrm{AGD})]^{+}$}

Charge $=1$ Multiplicity $=1$

$\mathrm{Cu}, 0,-0.1169289372,-0.1160138118,0.0147385679$

$\mathrm{N}, 0,2.8298291911,1.8346919213,1.3084406224$

$\mathrm{H}, 0,2.3688778784,2.7365976603,1.2919782283$

$\mathrm{H}, \mathrm{0}, 3.7237133403,1.9480660662,1.7752417407$

$\mathrm{N}, 0,3.0990916947,-0.464682597,-1.4455117802$

$\mathrm{H}, 0,3.7430845305,0.1361977122,-1.9539008411$

$\mathrm{C}, 0,2.7086254221,0.1303261684,-0.3779778022$

$\mathrm{N}, 0,3.0352653867,1.4038585527,-0.0012173794$

$\mathrm{H}, \mathrm{O}, 3.7661321985,1.847714107,-0.5411348262$

$\mathrm{N}, 0,1.8000234515,-0.5149076704,0.4897852167$

$\mathrm{H}, 0,1.9325341592,-0.180625168,1.4411054148$

$\mathrm{H}, 0,1.9473400982,-1.5162325531,0.4448510242$

$\mathrm{N}, 0,-1.933076219,-2.0343687067,0.4466261025$

$\mathrm{H}, \mathrm{O},-1.8091163458,-2.9190981862,-0.0311244662$ $\mathrm{H}, \mathrm{O},-2.073627134,-2.2253848872,1.4332144594$

$\mathrm{N}, 0,-3.0481229743,-1.3935385385,-0.0719275239$

C, $0,-2.9846328674,-0.0863208067,-0.441539486$

$\mathrm{N}, 0,-1.8535669549,0.5580164903,-0.4859610227$

$\mathrm{N}, 0,-4.190196367,0.4840930942,-0.7310674154$

$\mathrm{H}, \mathrm{O},-4.1521098598,1.3210646816,-1.2916602112$

$\mathrm{H}, \mathrm{O},-4.9454067589,-0.1386414628,-0.9758817545$

$\mathrm{H}, \mathrm{O},-1.9893704354,1.5061196032,-0.8111170431$

$\mathrm{H}, \mathrm{O},-3.9603434975,-1.786362669,0.1139261752$

\section{$[40]\left[\mathrm{Cu}\left(\mathrm{AG}_{\mathrm{D}}\right)_{2}\right]^{+} \cdot \mathrm{H}_{2} \mathrm{O}$}

Charge $=1$ Multiplicity $=1$

$\mathrm{Cu}, 0,-0.3674152373,-0.1518907173,-0.4266520551$ $\mathrm{N}, 0,1.5455615677,-2.2554594586,-0.5777074997$ $\mathrm{H}, 0,1.5493862647,-2.9256626524,0.1839772756$ $\mathrm{H}, \mathrm{O}, 1.5791754707,-2.7702965372,-1.4493410853$ $\mathrm{N}, 0,1.4147482709,0.4905771771,-0.0928278591$ $\mathrm{H}, 0,1.5556895906,1.4676020659,0.1482546188$ C, $0,2.5559506217,-0.1298518058,-0.1464398583$ $\mathrm{N}, 0,2.6661382222,-1.4422309418,-0.4921350224$ $\mathrm{H}, 0,3.5643669605,-1.8814871435,-0.3478315371$ $\mathrm{N}, 0,3.7479085349,0.4717186035,0.1582618305$ $\mathrm{H}, 0,3.7532123738,1.4766882862,0.075229055$ $\mathrm{H}, \mathrm{O}, 4.5815908352,0.0329132685,-0.2042341518$ $\mathrm{N}, 0,-2.1165464015,-0.1693327112,1.8525420971$ $\mathrm{H}, \mathrm{O},-1.9236909755,-0.7655386998,2.6479748544$ $\mathrm{H}, \mathrm{O},-2.3066676205,0.7655327823,2.1981532658$ $\mathrm{N}, 0,-3.2286727779,-0.6601641787,1.1848721775$ $C, 0,-3.2407190083,-0.7716378981,-0.170840432$ $\mathrm{N}, 0,-2.1750221958,-0.6080031201,-0.900809178$ $\mathrm{N}, 0,-4.4710563522,-1.0447830253,-0.7014817169$ $\mathrm{H}, \mathrm{O},-4.4605580839,-1.4591022838,-1.6207578854$ $\mathrm{H}, 0,-5.1523692842,-1.4553507681,-0.0803752026$ $\mathrm{H}, \mathrm{O},-2.3934489876,-0.7764952859,-1.8747382467$ $\mathrm{H}, 0,-4.1268861617,-0.6074861087,1.6459619145$ $0,0,1.9537728084,3.4693184076,0.6310660992$ $\mathrm{H}, 0,1.5337889847,4.1201652537,0.0619001081$ $\mathrm{H}, \mathrm{O}, 2.8926145804,3.6614964913,0.553660434$
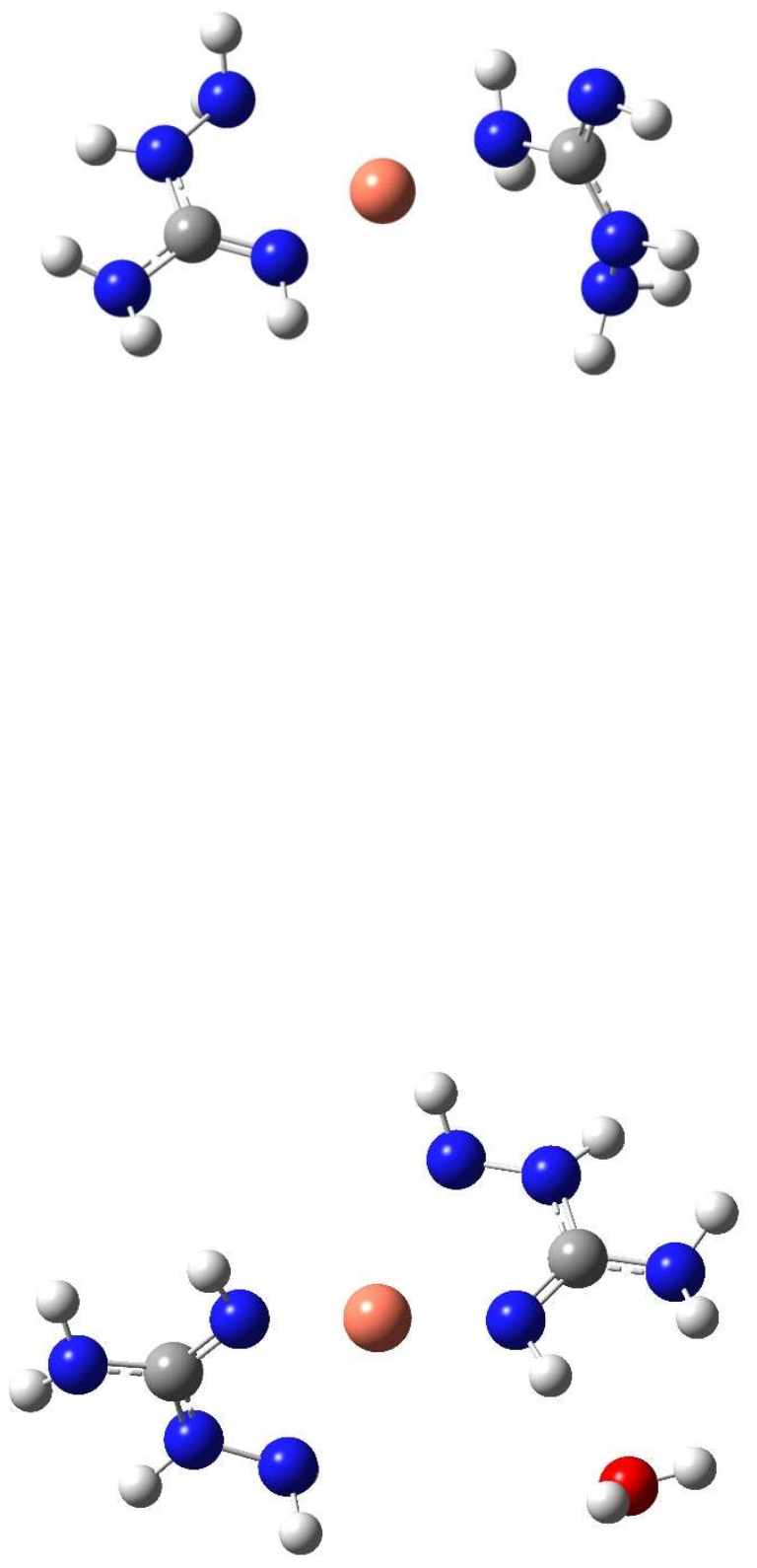


\section{$[41]\left[\mathrm{Cu}\left(\mathrm{AGD}_{\mathrm{D}}\right)_{2}\right]^{+}$}

Charge $=1$ Multiplicity $=1$

$\mathrm{Cu}, 0,-0.0183657393,-0.1265972382,-0.0523009779$

$\mathrm{N}, 0,1.9271576943,0.6730051433,-2.0997210807$

$\mathrm{H}, 0,2.0275480014,0.0320771884,-2.879883273$

$\mathrm{H}, \mathrm{O}, 1.882898295,1.6145866898,-2.4701932506$

$\mathrm{N}, 0,1.804398248,-0.3274746788,0.5152437511$

$\mathrm{H}, 0,1.965203621,-0.7218114373,1.4334831892$

$\mathrm{C}, 0,2.9401922207,-0.0102816021,-0.0388748672$

$\mathrm{N}, 0,3.029583795,0.5706663659,-1.2650369214$

$\mathrm{H}, 0,3.9499692741,0.6311175442,-1.6787868048$

$\mathrm{N}, 0,4.1481722994,-0.2572366006,0.5528375019$

$\mathrm{H}, 0,4.1265762654,-0.3631798379,1.5551711324$

$\mathrm{H}, \mathrm{O}, 4.9360162917,0.2792423436,0.2208449452$

$\mathrm{N}, 0,-1.9478704851,-0.951917149,1.9892784095$

$\mathrm{H}, \mathrm{O},-1.9773420496,-1.9011964403,2.3411564555$ $\mathrm{H}, \mathrm{O},-1.9558820498,-0.3204602992,2.783531346$

$\mathrm{N}, 0,-3.0694980936,-0.7310988719,1.2041368154$

$\mathrm{C}, 0,-2.9780404384,-0.136787408,-0.0156614284$ $\mathrm{N}, 0,-1.8417950785,0.0949462414,-0.6097554111$ $\mathrm{N}, 0,-4.1828383379,0.2202298627,-0.5552248337$ $\mathrm{H}, \mathrm{O},-4.1867787908,0.3562055044,-1.5539980831$ $\mathrm{H}, \mathrm{O},-4.9981555232,-0.2650369479,-0.2109339757$ $\mathrm{H}, \mathrm{O},-2.0047025805,0.5058327087,-1.5203733627$ $\mathrm{H}, \mathrm{O},-3.9730050591,-0.7106582413,1.6571947841$

\section{$[42]\left[\mathrm{Cu}(\mathrm{AGD})_{2}\right]^{+}$(mirror image)}

Charge $=1$ Multiplicity $=1$

$\mathrm{Cu}, 0,0.0020468443,-1.0133792378,0.0524731832$ $\mathrm{N}, 0,-1.4889148088,1.1357669508,-1.09960462$ $\mathrm{H}, \mathrm{O},-1.2700450005,1.9876908624,-0.5927247357$ $\mathrm{H}, \mathrm{O},-1.4983994275,1.350280956,-2.0891345883$ $\mathrm{N}, 0,-1.8774175058,-1.1488265167,0.4451178149$ $\mathrm{H}, \mathrm{O},-2.19641997,-1.9128881333,1.0275131784$ C, $0,-2.8814486549,-0.3961229086,0.097778641$ $\mathrm{N}, 0,-2.7446485385,0.6742984831,-0.7321767151$ $\mathrm{H}, \mathrm{O},-3.51896473,1.3229787416,-0.7659630269$ $\mathrm{N}, 0,-4.1554712538,-0.5908383291,0.5543776727$ $\mathrm{H}, \mathrm{O},-4.3552330299,-1.5182619499,0.8953893926$ $\mathrm{H}, \mathrm{O},-4.902887917,-0.1902779082,0.0069658501$ $\mathrm{N}, 0,1.5266847686,1.0917507543,1.1611987715$ $\mathrm{H}, 0,1.2228533585,2.0108211112,0.862457467$ $\mathrm{H}, 0,1.6779408286,1.118583526,2.1639586656$ $\mathrm{N}, 0,2.7142844013,0.7761579531,0.5172011473$ C, $0,2.8743943135,-0.4243060234,-0.1033392444$ $\mathrm{N}, 0,1.8764243143,-1.2294929675,-0.331442725$ $\mathrm{N}, 0,4.1636832114,-0.7181878846,-0.4458496244$ $\mathrm{H}, \mathrm{O}, 4.2735744242,-1.3873139206,-1.1918421105$ $\mathrm{H}, 0,4.8186917099,0.0482165996,-0.4838176285$ $\mathrm{H}, \mathrm{O}, 2.1950624849,-2.0572786433,-0.818779249$ $\mathrm{H}, \mathrm{O}, 3.5522871772,1.268431485,0.7934554835$
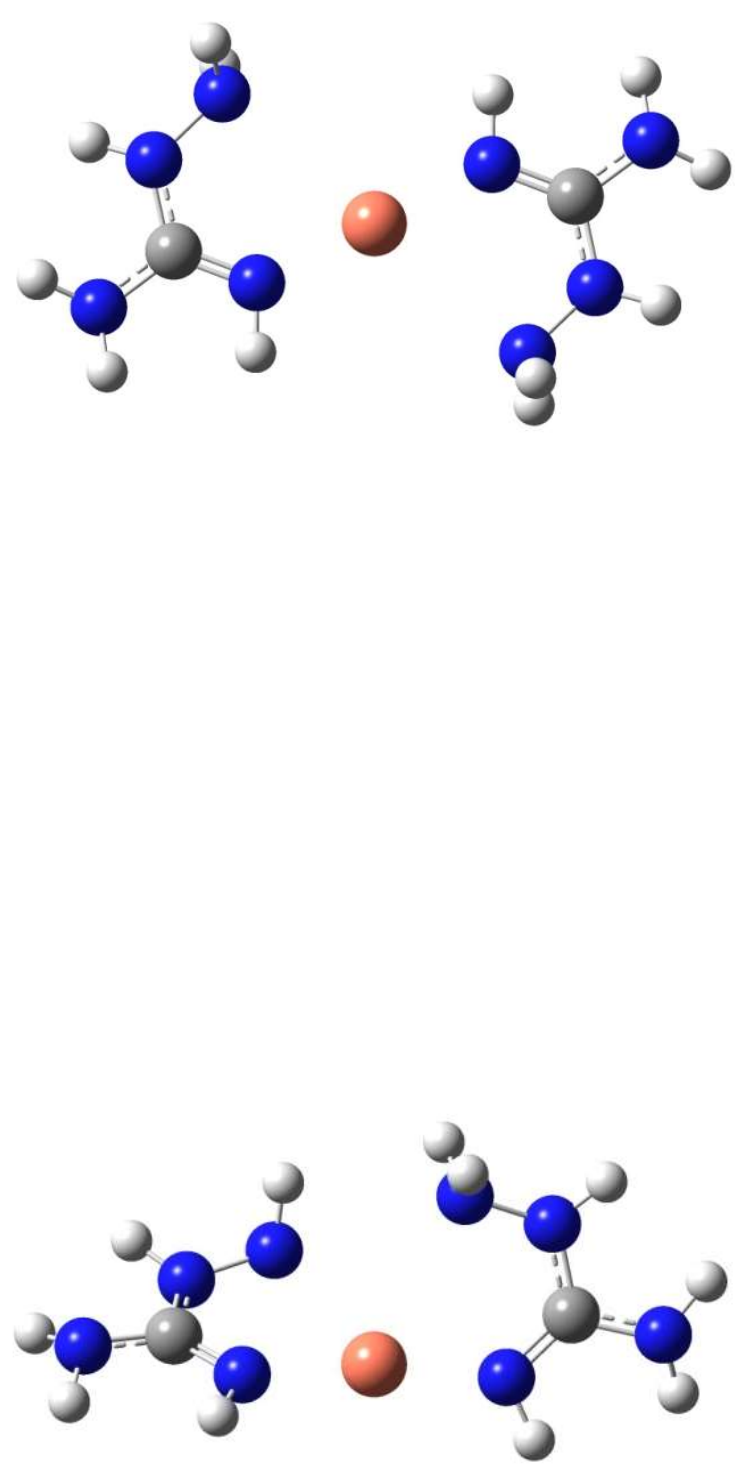


\section{$[43]\left[\mathrm{Cu}\left(\mathrm{H}_{2} \mathrm{O}\right)_{2}\right]^{+} \cdot 2 \mathrm{H}_{2} \mathrm{O}$}

Charge $=1$ Multiplicity $=1$

$\mathrm{Cu}, 0,-0.0132023766,0.0390416648,0.1187538989$ $0,0,1.6237839754,1.1690451124,0.0684467062$ $\mathrm{H}, 0,2.4331140325,0.6197893607,0.145866631$ $\mathrm{H}, 0,1.6602343574,1.791023754,0.8009138957$ $0,0,-1.6507503775,-1.0917297672,0.1586077716$ $\mathrm{H}, 0,-1.722276289,-1.6405293264,-0.6275897492$ $\mathrm{H}, \mathrm{O},-2.4553347856,-0.5299878067,0.1630250234$ $0,0,-3.8242884985,0.573493956,0.2984795494$ $\mathrm{H}, \mathrm{O},-4.6588723871,0.1190147586,0.4472946494$ $0,0,3.810180471,-0.4798001348,0.1544823027$ $\mathrm{H}, 0,4.6037869629,-0.104042406,-0.2377611046$ $\mathrm{H}, 0,4.0799224595,-0.737508436,1.040930654$ $\mathrm{H}, \mathrm{0},-3.9662759944,1.0765675106,-0.5089523486$

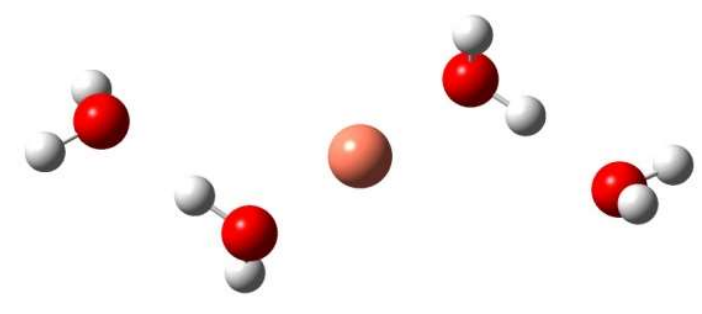

\section{$[44]\left[\mathrm{Cu}\left(\mathrm{H}_{2} \mathrm{O}\right)_{4}\right]^{2+}$}

Charge $=2$ Multiplicity $=2$

$\mathrm{Cu}, 0,0.0113164963,-0.0062243347,0.0097231411$ $0,0,1.9140133948,-0.7145563283,0.3020305878$ $\mathrm{H}, \mathrm{0}, 1.9807349509,-1.636489633,0.0252958416$ $\mathrm{H}, 0,2.5433892571,-0.2369565749,-0.2518414035$ $0,0,-0.7979071443,-1.8702290581,0.1589600187$ $\mathrm{H}, 0,-1.5974079542,-1.9260361675,-0.3784848832$ $\mathrm{H}, \mathrm{O},-0.2069465222,-2.5451772268,-0.1950481928$ $0,0,0.7353863288,1.8926155175,-0.3316553127$ $\mathrm{H}, 0,0.1919874746,2.5408451741,0.1320904315$ $\mathrm{H}, 0,1.618310247,1.9864520972,0.0456218549$ $0,0,-1.9435517268,0.6325979124,-0.1147636595$ $\mathrm{H}, \mathrm{O},-2.4500078173,0.291060506,0.6315668071$ $\mathrm{H}, 0,-1.9978459848,1.5928211163,-0.039158231$

\section{[45] $\mathrm{AGH}^{+}$}

\footnotetext{
Charge $=1$ Multiplicity $=1$

C, $0,-0.5010286928,0.0103715891,0.0041964808$

$\mathrm{N}, 0,0.6595667723,-0.6491621382,-0.0531533786$ $\mathrm{N}, 0,1.8562754498,0.0468278718,-0.1014158253$ $\mathrm{N}, 0,-0.5075005806,1.3327690572,0.0446957836$ $\mathrm{N}, 0,-1.6384791073,-0.6837524445,-0.0009224011$ $\mathrm{H}, \mathrm{O},-2.5055459869,-0.208278755,0.1898411662$ $\mathrm{H}, \mathrm{O},-1.6091955947,-1.6812730878,0.1392147922$ $\mathrm{H}, 0,0.6443556166,-1.6593787368,-0.1034638027$ $\mathrm{H}, 0,2.3442752974,-0.1900499848,-0.9581074148$ $\mathrm{H}, \mathrm{O}, 0.3719683465,1.8220891832,-0.0103350135$ $\mathrm{H}, 0,2.4337630924,-0.2336493311,0.6831292744$ $\mathrm{H}, \mathrm{O},-1.3767643027,1.8395189069,0.0686572287$
}

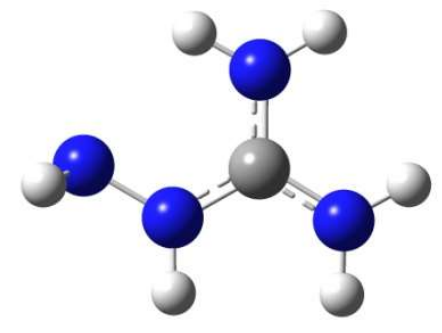




\section{[46] $\left(\mathrm{AGH}^{+}\right)_{2}$}

Charge $=2$ Multiplicity $=1$

$\mathrm{N}, 0,-1.218931,1.519416,0.375765$

$\mathrm{H}, 0,-1.086277,1.841759,1.328854$

$\mathrm{H}, 0,-1.071501,2.309877,-0.243675$

$\mathrm{N}, 0,-1.829234,-1.056084,-0.278577$

$\mathrm{H}, \mathrm{0},-2.052223,-2.010715,-0.509097$

C, $0,-2.803083,-0.177424,-0.107891$

$\mathrm{N}, 0,-2.528001,1.085404,0.230525$

$\mathrm{H}, 0,-3.294371,1.731824,0.36387$

$\mathrm{N}, 0,-4.076288,-0.527155,-0.300828$

$\mathrm{H}, \mathrm{O},-4.307904,-1.505976,-0.359579$

$\mathrm{H}, 0,-4.809637,0.105152,-0.021409$

$\mathrm{N}, 0,1.21907,-1.519581,0.375488$

$\mathrm{H}, 0,1.086327,-1.842034,1.328526$

$\mathrm{H}, 0,1.071823,-2.309989,-0.244064$

$\mathrm{N}, 0,2.528099,-1.08538,0.23049$

$\mathrm{C}, 0,2.803049,0.177468,-0.107958$

$\mathrm{N}, 0,1.829076,1.055918,-0.27906$

$\mathrm{N}, 0,4.076237,0.527415,-0.300538$

$\mathrm{H}, 0,4.307731,1.506246,-0.359537$

$\mathrm{H}, 0,4.809649,-0.104789,-0.021056$

$\mathrm{H}, 0,2.05197,2.010623,-0.509363$

$\mathrm{H}, 0,3.294549,-1.731661,0.36406$

$\mathrm{H}, 0,0.867011,0.800996,-0.097865$

$\mathrm{H}, 0,-0.867142,-0.801247,-0.097425$

\section{[47] $\mathrm{AG}_{\mathrm{A}}$}

Charge $=0$ Multiplicity $=1$

$\mathrm{N}, 0,1.8464653399,0.0082114418,0.0387940031$ $\mathrm{H}, \mathrm{O}, 2.4963342272,-0.3649145358,-0.6414615986$ $\mathrm{H}, 0,2.2224788455,-0.1735579737,0.9638701366$ $\mathrm{N}, 0,0.6177828001,-0.6300332508,-0.116562949$ C, $0,-0.526230913,0.1390255661,-0.0093279574$ $\mathrm{N}, 0,-0.5691436878,1.4259681856,-0.0205693271$ $\mathrm{N}, 0,-1.6711096016,-0.6152804174,0.1230851829$ $\mathrm{H}, 0,-2.5085659506,-0.111951078,-0.1244106458$ $\mathrm{H}, 0,-1.6367674705,-1.5387667349,-0.2832872472$ $\mathrm{H}, 0,0.3922483982,1.7578824647,-0.0533177263$ $\mathrm{H}, 0,0.5471860127,-1.5721186676,0.2458471287$

\section{[48] $\mathrm{AG}_{\mathrm{B}}$}

Charge $=0$ Multiplicity $=1$

$\mathrm{N}, 0,-1.7987343346,0.1121860392,0.1126460421$

$\mathrm{H}, \mathrm{O},-2.1729518457,-0.1643587059,1.0148517934$

$\mathrm{H}, \mathrm{O},-2.5092961613,-0.0693211915,-0.5852784915$

$\mathrm{N}, 0,-0.6642512732,-0.6448141806,-0.19115797$

C, $0,0.5856878065,-0.0780974696,-0.0289725074$

$\mathrm{N}, 0,1.606105758,-0.8643412579,0.0917519139$

$\mathrm{N}, 0,0.6134976627,1.2867014432,-0.113114896$

$\mathrm{H}, \mathrm{O}, 1.4288204047,1.7263122349,0.282401736$

$\mathrm{H}, \mathrm{O},-0.2651006834,1.7429015534,0.0879110104$

$\mathrm{H}, \mathrm{O}, 2.4535264177,-0.3051184255,0.1148813861$

$\mathrm{H}, 0,-0.6868017514,-1.6238830396,0.0599789829$
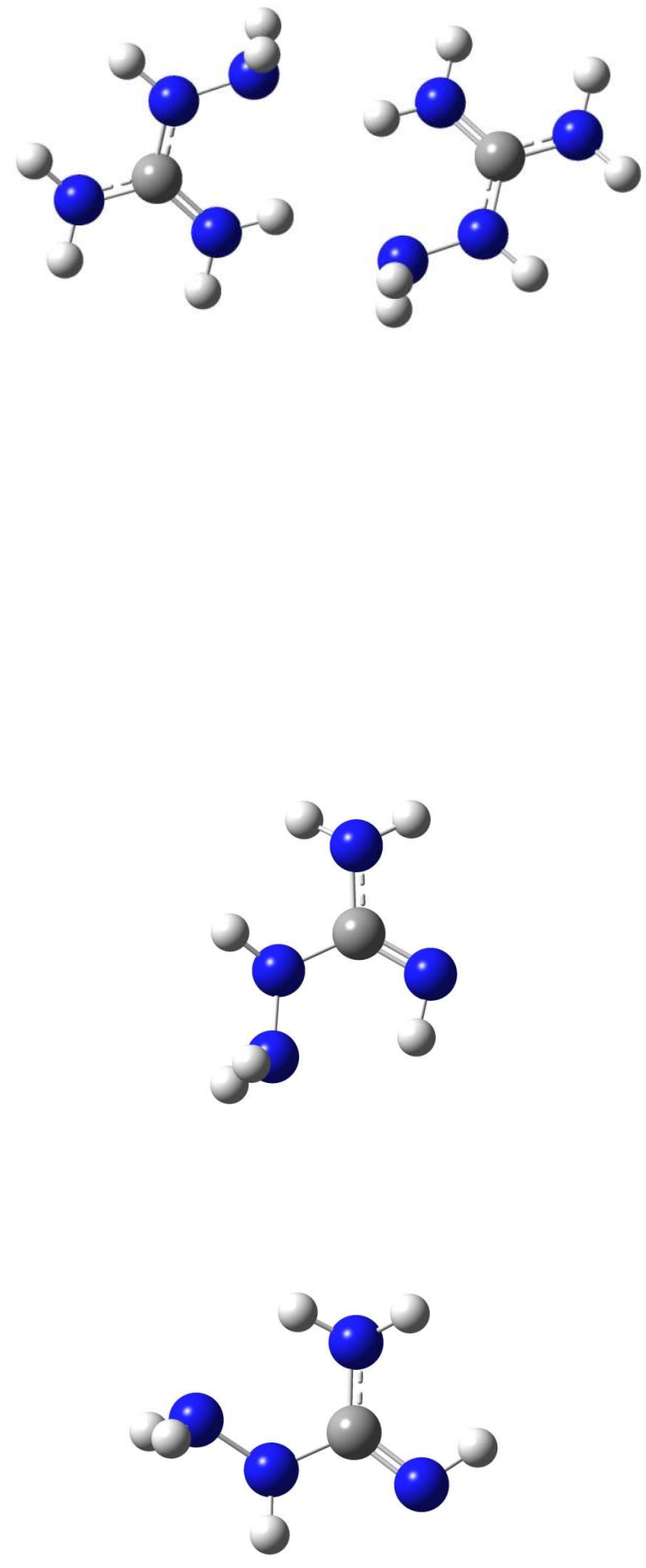


\section{[49] AGc}

Charge $=0$ Multiplicity $=1$

$\mathrm{N}, 0,-1.7944358785,0.0754541235,0.0928035693$

$\mathrm{H}, \mathrm{O},-2.1868665652,-0.1996099418,0.9873714806$

$\mathrm{H}, \mathrm{O},-2.4776489824,-0.135758399,-0.6242538603$

$\mathrm{N}, 0,-0.6287676303,-0.6506976282,-0.164206504$

$\mathrm{C}, 0,0.6030744705,-0.0388197837,-0.0278925915$

$\mathrm{N}, 0,1.719676109,-0.6868188565,0.0663805611$

$\mathrm{N}, 0,0.5792398102,1.3247900026,-0.1057501873$

$\mathrm{H}, \mathrm{O}, 1.3992846386,1.7800780394,0.2595693052$

$\mathrm{H}, \mathrm{O},-0.303607857,1.7508774261,0.1333241397$

$\mathrm{H}, \mathrm{O}, 1.5252363258,-1.6844579535,0.0815167435$

$\mathrm{H}, 0,-0.6343074407,-1.6291670291,0.0913723436$
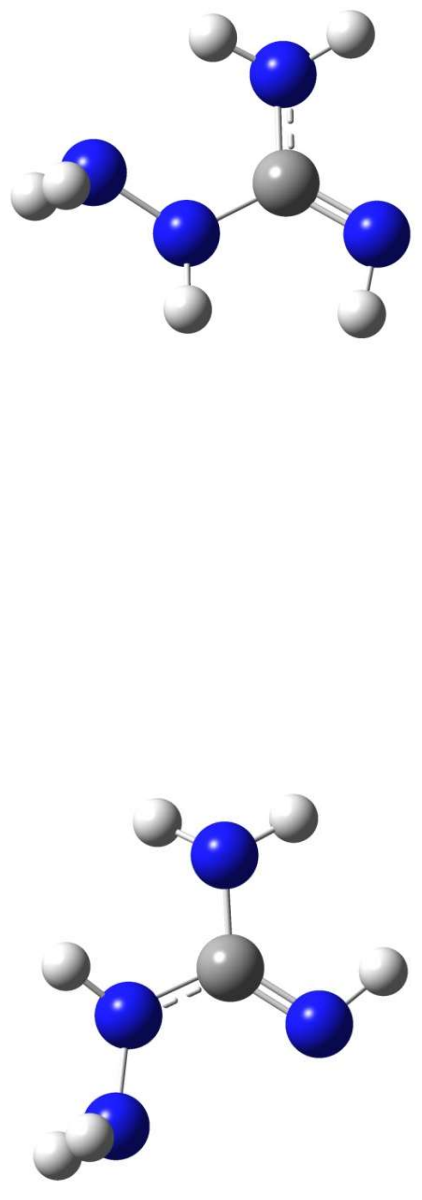

Charge $=0$ Multiplicity $=1$

$\mathrm{C}, 0,-0.506095489,0.1220613005,-0.0120571794$

$\mathrm{N}, 0,0.6881725629,-0.558049556,-0.1030855864$

$\mathrm{N}, 0,1.8972681979,0.1195469342,0.0309539592$

$\mathrm{N}, 0,-0.5801689448,1.4095510217,-0.0327642233$

$\mathrm{N}, 0,-1.5833030443,-0.7360492234,0.1261238479$

$\mathrm{H}, \mathrm{O},-2.4647134969,-0.3298073653,-0.1481545736$

$\mathrm{H}, \mathrm{O},-1.4585255775,-1.6642772817,-0.2524295138$

$\mathrm{H}, 0,0.6674604819,-1.5122344587,0.2309693211$

$\mathrm{H}, 0,2.5626206935,-0.2878842635,-0.6137245439$

$\mathrm{H}, 0,-1.5610607694,1.6722294862,-0.0074410442$

$\mathrm{H}, \mathrm{O}, 2.2618503855,-0.003345594,0.9703365366$

\section{[51] $\left(\mathrm{AG}_{\mathrm{A}}\right)_{2}$}

Charge $=0$ Multiplicity $=1$

$\mathrm{N}, 0,-4.020652936,-1.748896825,0.3190639115$

$\mathrm{H}, \mathrm{O},-4.4500193697,-2.1191132734,-0.5228365237$

$\mathrm{H}, 0,-4.7445630171,-1.6475462697,1.0192030261$

$\mathrm{N}, 0,-3.4617027667,-0.4974637428,0.0659026823$

C, $0,-2.0890327305,-0.4221790266,-0.0937323029$

$\mathrm{N}, 0,-1.2573826978,-1.3606428701,0.2210223033$

$\mathrm{N}, 0,-1.659140806,0.7626460395,-0.627906569$

$\mathrm{H}, \mathrm{O},-0.6825227584,0.9935778536,-0.4354344087$

$\mathrm{H}, \mathrm{O},-2.2940119831,1.5421063587,-0.5480221323$

$\mathrm{H}, \mathrm{O},-1.8047530723,-2.1580043518,0.5351154072$

$\mathrm{H}, \mathrm{0},-4.0056341463,0.1343986784,-0.5076301925$

$\mathrm{N}, 0,4.049024227,1.7109298464,-0.127142485$

$\mathrm{H}, 0,4.4523229376,1.9133105167,-1.0363546326$

$\mathrm{H}, 0,4.79381376,1.7420129009,0.557585909$

$\mathrm{N}, 0,3.4867016069,0.4353389813,-0.1215295984$

C, $0,2.1101744225,0.3353120206,-0.2264163245$

$\mathrm{N}, 0,1.2852616808,1.3186099701,-0.0724572133$

$\mathrm{N}, 0,1.668429218,-0.92809287,-0.5144220093$

$\mathrm{H}, 0,0.6984917822,-1.115678578,-0.2532032455$

$\mathrm{H}, \mathrm{O}, 2.3074137564,-1.6799436647,-0.3059232277$

$\mathrm{H}, 0,1.8386079806,2.1597621191,0.0698480681$

$\mathrm{H}, 0,4.0153586818,-0.2943307232,-0.5822195723$

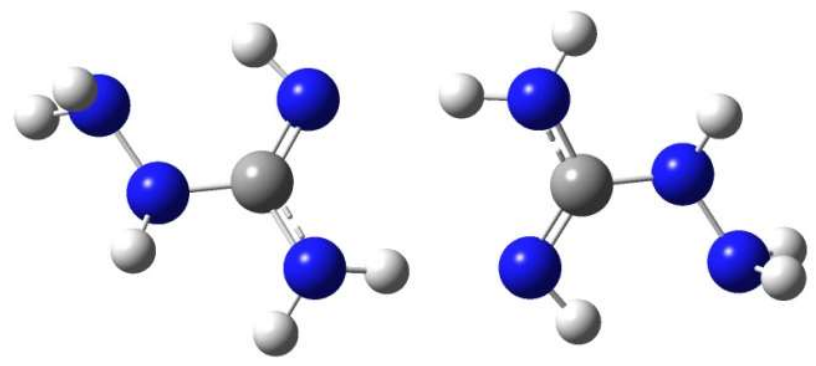




\section{[52] $\left(\mathrm{AG}_{\mathrm{B}}\right)_{2}$}

Charge $=0$ Multiplicity $=1$

$\mathrm{N}, 0,0.0036465662,1.5327630802,-2.8789661778$ $\mathrm{H}, \mathrm{O},-0.7988541118,1.6510824229,-3.490394877$ $\mathrm{H}, \mathrm{O}, 0.1457813673,2.4074409487,-2.3887731341$ $\mathrm{N}, 0,2.3452408208,-0.3610122823,-4.7399037867$ $\mathrm{H}, 0,2.6688786597,-1.3166925718,-4.6247572131$ C, $0,1.5966284047,-0.0456935278,-3.731925187$ $\mathrm{N}, 0,1.1451177788,1.2581312481,-3.6389253776$ $\mathrm{H}, 0,1.2331074336,1.7586299531,-4.5129396315$ $\mathrm{N}, 0,1.2758120396,-0.8506610417,-2.6762951837$ $\mathrm{H}, \mathrm{O}, 1.3698517748,-1.8393942295,-2.8432403299$ $\mathrm{H}, 0,0.4724158082,-0.5829318964,-2.121982024$ $\mathrm{N}, 0,-0.8091176159,-1.2599693385,-0.2444645823$ $\mathrm{H}, \mathrm{0},-0.6787133298,-2.2154786216,-0.5542032997$ $\mathrm{H}, \mathrm{O},-0.0994703167,-1.0648709462,0.4555996123$ $\mathrm{N}, 0,-2.0829355855,-1.1469998487,0.3195838355$ $\mathrm{C}, 0,-2.8202710287,0.0022157109,0.1069118349$ $\mathrm{N}, 0,-3.7829115444,0.271153242,0.929751189$ $\mathrm{N}, 0,-2.5065032098,0.6955537319,-1.0279269837$ $\mathrm{H}, \mathrm{O},-2.8498043415,1.6420253503,-1.0572765395$ $\mathrm{H}, \mathrm{O},-1.5721020737,0.5678153972,-1.3954935108$ $\mathrm{H}, \mathrm{O},-4.2798001702,1.0924052662,0.5987636415$ $\mathrm{H}, \mathrm{0},-2.1995912957,-1.516934277,1.2530842155$

\section{[53] (AGc)2}

Charge $=0$ Multiplicity $=1$

$\mathrm{N}, 0,0.2775020528,-0.9119067673,0.2222657334$ $\mathrm{H}, 0,0.0256160146,-1.025641505,1.1995101818$ $\mathrm{H}, \mathrm{O},-0.5114675014,-1.2181175653,-0.334840712$ $\mathrm{N}, 0,1.3775356622,-1.7190906774,-0.0832282093$ $\mathrm{C}, 0,2.6366530794,-1.1591791699,-0.193491557$ $\mathrm{N}, 0,3.7309156253,-1.8508669113,-0.146768277$ $\mathrm{N}, 0,2.6601949427,0.1781571896,-0.4637935177$ $\mathrm{H}, \mathrm{O}, 3.5531511189,0.6166259298,-0.3089602411$ $\mathrm{H}, 0,1.8564219523,0.7211649756,-0.1784392865$ $\mathrm{H}, 0,3.5039243795,-2.824313289,0.0365257543$ $\mathrm{H}, \mathrm{O}, 1.3769782128,-2.6498418134,0.3121342908$ $\mathrm{N}, 0,1.116520035,2.8894187583,0.3658480126$ $\mathrm{H}, 0,1.3946100213,2.924410455,1.3419845289$ $\mathrm{H}, 0,1.8906809689,3.2402416807,-0.1857081532$ $\mathrm{N}, 0,0.0096665515,3.7175727194,0.1559247867$ $\mathrm{C}, 0,-1.2526517522,3.1671164863,0.0351370766$ $\mathrm{N}, 0,-2.3444188783,3.8516790333,0.1672300321$ $\mathrm{N}, 0,-1.2851332632,1.8558644712,-0.341277037$ $\mathrm{H}, \mathrm{O},-2.1741428685,1.4055338814,-0.1982327919$ $\mathrm{H}, 0,-0.4745817346,1.2924073894,-0.1225142719$ $\mathrm{H}, \mathrm{O},-2.1113730088,4.8074200657,0.4221211929$ $\mathrm{H}, \mathrm{O}, \mathrm{0} .0220736396,4.6135267328,0.6246724846$
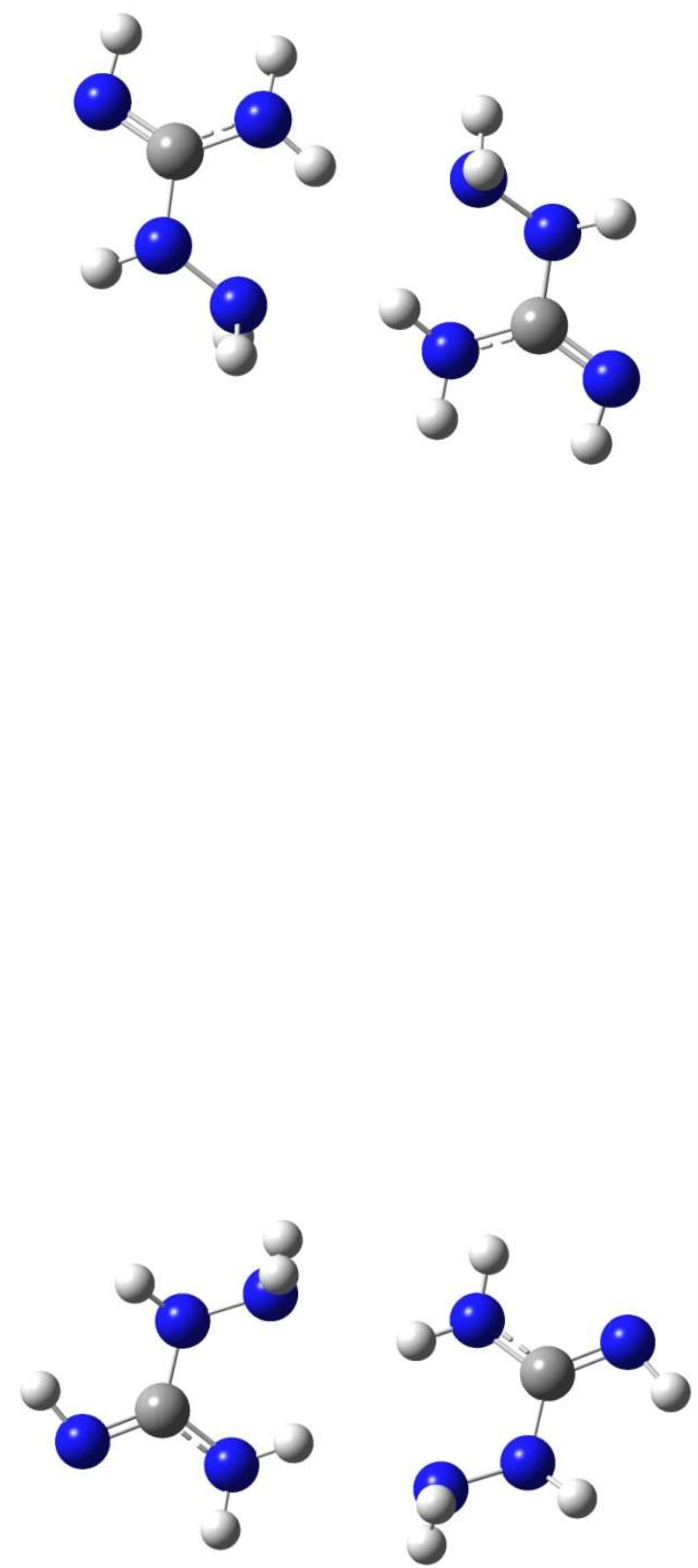


\section{[54] (AGD)2}

Charge $=0$ Multiplicity $=1$

$\mathrm{N}, 0,0.7034348487,2.7829182049,-1.2310753763$

$\mathrm{H}, \mathrm{O},-0.1905803465,2.2896414494,-1.1690405396$ $\mathrm{H}, 0,0.5119731482,3.7330851357,-1.5238062318$ $\mathrm{N}, 0,2.9370260697,1.2219437442,-0.6160961888$ $\mathrm{H}, 0,3.6931826929,0.5438417029,-0.5991381836$ $\mathrm{N}, 0,1.511355455,2.1906388015,-2.2041900866$ $\mathrm{H}, \mathrm{O}, 1.0385775096,1.9342139395,-3.0614767979$ $\mathrm{N}, 0,2.9387567272,0.5281382045,-2.8832221585$ $\mathrm{H}, 0,3.8676409707,0.155257555,-2.7611164143$ $\mathrm{H}, 0,2.7971776472,0.9116333152,-3.8068309366$ $\mathrm{N}, 0,-0.6398816287,-0.4374727626,-1.9042311155$ $\mathrm{H}, \mathrm{0},-0.6114544358,-1.1531659794,-2.6193443814$ $\mathrm{H}, \mathrm{O}, 0.2303896004,-0.489776392,-1.38124825$ $\mathrm{N}, 0,-1.7210145739,-0.7108867362,-1.0673086722$ $\mathrm{C}, 0,-2.3729777174,0.3282238554,-0.4409344837$ $\mathrm{N}, 0,-2.1718366516,1.5625815435,-0.7599477336$ $\mathrm{N}, 0,-3.2241978077,-0.1085811495,0.5561059075$ $\mathrm{H}, \mathrm{O},-3.947490465,0.5531455037,0.7928279634$ $\mathrm{H}, \mathrm{O},-3.5809785407,-1.0477534099,0.4500380808$ $\mathrm{H}, 0,-2.7972195986,2.1441650882,-0.2097453393$ $\mathrm{H}, 0,-1.6661283755,-1.5643564626,-0.5265743363$ C, $0,2.4981063316,1.3052206087,-1.8266256358$

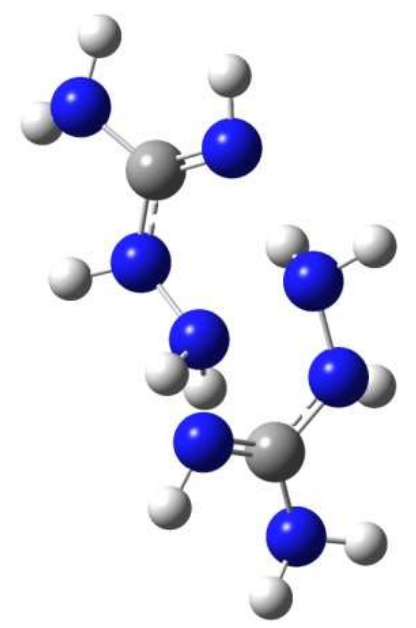

\section{[55] (AGc) $(\mathrm{AGD})$}

Charge $=0$ Multiplicity $=1$ $\mathrm{N}, 0,0.7115398163,0.4095501466,0.3815920986$ $\mathrm{H}, \mathrm{O}, 0.2536583839,0.8783571502,-0.39529184$ $\mathrm{H}, 0,0.2621273551,0.7165106534,1.236110442$ $\mathrm{N}, 0,4.2580264654,0.2152845384,-0.2352377891$ $\mathrm{H}, 0,4.3779939906,1.1979175912,-0.0058590078$ $\mathrm{C}, 0,3.0188182103,-0.1139262869,-0.054344493$ $\mathrm{N}, 0,2.0623920812,0.7545667599,0.433375294$ $\mathrm{H}, 0,2.2938457325,1.7339986366,0.3303640323$ $\mathrm{N}, 0,2.5749172274,-1.3727270486,-0.3379209277$ $\mathrm{H}, 0,1.6861133238,-1.6293560552,0.0616699069$ $\mathrm{H}, 0,3.285085214,-2.0858449457,-0.3487798144$ $\mathrm{N}, 0,-1.6586148412,-1.763733656,-0.3161707839$ $\mathrm{H}, 0,-1.9405010997,-2.620489787,0.1428704187$ $\mathrm{H}, 0,-0.6584855765,-1.6439109077,-0.1745154919$ $\mathrm{N}, 0,-2.3386983172,-0.7070307616,0.291290792$ $\mathrm{C}, 0,-2.6725127163,0.4051459736,-0.4549298929$ $\mathrm{N}, 0,-2.6456525954,0.4127553571,-1.743696476$ $\mathrm{N}, 0,-3.0114081282,1.4837225126,0.3418373964$ $\mathrm{H}, 0,-3.5879985996,2.1692595123,-0.1218566$ $\mathrm{H}, \mathrm{O},-3.3512700204,1.2602019472,1.2665625634$ $\mathrm{H}, 0,-2.9835761297,1.3156299645,-2.0641676002$ $\mathrm{H}, 0,-2.0547937764,-0.4948112949,1.2399587728$

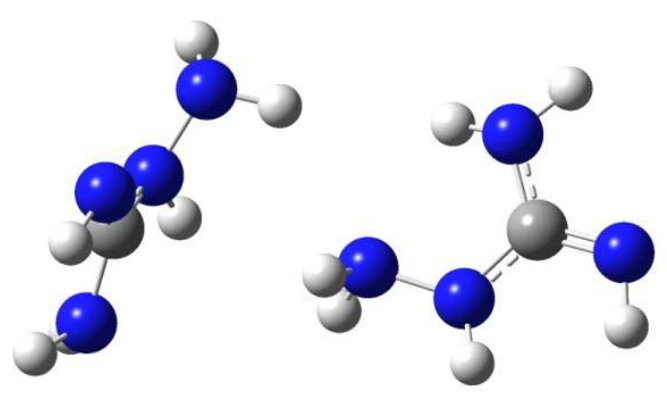

